\title{
Critical Configurations and Tube of Typical Trajectories for the Potts and Ising Models with Zero External Field
}

\author{
Gianmarco Bet ${ }^{1} \cdot$ Anna Gallo ${ }^{1}$. Francesca R. Nardi ${ }^{1,2}$ (D
}

Received: 13 February 2021 / Accepted: 16 August 2021 / Published online: 2 September 2021

(c) The Author(s) 2021

\begin{abstract}
We consider the ferromagnetic $q$-state Potts model with zero external field in a finite volume evolving according to Glauber-type dynamics described by the Metropolis algorithm in the low temperature asymptotic limit. Our analysis concerns the multi-spin system that has $q$ stable equilibria. Focusing on grid graphs with periodic boundary conditions, we study the tunneling between two stable states and from one stable state to the set of all other stable states. In both cases we identify the set of gates for the transition and prove that this set has to be crossed with high probability during the transition. Moreover, we identify the tube of typical paths and prove that the probability to deviate from it during the transition is exponentially small.
\end{abstract}

Keywords Potts model · Ising model · Glauber dynamics · Metastability · Tunnelling behaviour · Critical droplet · Tube of typical trajectories · Gate $\cdot$ Large deviations

Mathematics Subject Classification Primary 60K35 $\cdot 82 \mathrm{C} 20 \cdot$ Secondary 60J10 $\cdot 82 \mathrm{C} 22$

Communicated by Alessandro Giuliani.

The research of Francesca R. Nardi was partially supported by the NWO Gravitation Grant 024.002.003-NETWORKS and by the PRIN Grant 20155PAWZB "Large Scale Random Structures".

Francesca R. Nardi

francescaromana.nardi@unifi.it; f.r.nardi@tue.nl

Gianmarco Bet

gianmarco.bet@unifi.it

Anna Gallo

anna.gallo1@stud.unifi.it

1 Dipartimento di Matematica e Informatica 'Ulisse Dini', Università degli Studi di Firenze, Viale Morgagni 65, 50134 Firenze, Italia

2 Department of Mathematics and Computer Science, Eindhoven University of Technology, 5600MB, Eindhoven, The Netherlands 


\section{List of symbols}

$\begin{array}{ll}\text { Symbol } & \text { Meaning } \\ H & \text { Hamiltonian function } \\ \mathcal{X} & \text { State space } \\ \mathcal{X}^{s} & \text { Set of global minima }\end{array}$

$\mathcal{X}^{m}$

Set of metastable configurations

$S$

Set of all possible spin values

$N_{s}(\sigma) \quad$ Number of spins $s$ in configuration $\sigma$

$\mathcal{X}(r, s) \quad$ Set of configurations with all spins either $r$ or $s$

$\Omega_{\sigma, \sigma^{\prime}}$ Set of all the paths from $\sigma$ to $\sigma^{\prime}$

$\Omega_{\sigma, \sigma^{\prime}}^{o p t}$

$\Phi_{\omega}$

$\Phi\left(\sigma, \sigma^{\prime}\right)$

$\tau_{\mathcal{A}}^{\sigma}$

$\mathcal{S}\left(\sigma, \sigma^{\prime}\right)$

Set of all the optimal paths from $\sigma$ to $\sigma^{\prime}$

$\max _{i=0, \ldots, n} H\left(\omega_{i}\right)$, height of the path $\omega$

$\mathcal{W}\left(\sigma, \sigma^{\prime}\right)$

$\min _{\omega: \sigma \rightarrow \sigma^{\prime}} \Phi_{\omega}$, communication height between $\sigma$ and $\sigma^{\prime}$

$\mathcal{W}_{\text {RES }}\left(\mathbf{s}, \mathbf{s}^{\prime}\right) \quad$ Restricted-gate for the transition from $\mathbf{s} \in \mathcal{X}^{s}$ and $\mathbf{s}^{\prime} \in \mathcal{X}^{s} \backslash\{\mathbf{s}\}$ following a path that does not intersect $\mathcal{X}^{s} \backslash\left\{\mathbf{s}, \mathbf{s}^{\prime}\right\}$

$\mathcal{G}\left(\sigma, \sigma^{\prime}\right) \quad$ Union of all minimal gates for the transition from $\sigma$ to $\sigma^{\prime}$

$\mathcal{F}\left(\mathbf{s}, \mathbf{s}^{\prime}\right) \quad$ Union of all minimal restricted-gate for the transition $\sigma \rightarrow \sigma^{\prime}$

$\mathcal{C}_{\mathcal{A}}(\sigma) \quad$ Initial cycle for the transition $\sigma \rightarrow \mathcal{A}$

$\mathscr{C}(\mathcal{X})$

$\mathcal{M}(\mathcal{A})$

Set of all the cycles and extended cycles of $\mathcal{X}$

$\mathcal{B}(\mathcal{C})$

Collection of maximal cycles and extended cycles that partitions $\mathcal{A} \subset \mathcal{X}$

$\partial^{n p} \mathcal{C}$

Principal boundary of the cycle $\mathcal{C}$

$T_{\mathcal{A}}(\sigma)$

Non-principal boundary of the cycle $\mathcal{C}$

$\mathfrak{T}_{\mathcal{A}}(\sigma)$

$\left\{\eta \in \mathcal{X} \mid \exists \omega \in \Omega_{\sigma, A}^{\mathrm{vtj}}: \eta \in \omega\right\}$, tube of typical paths from $\sigma$ to $\mathcal{A}$

$\left\{\mathcal{C} \in \mathcal{M}\left(\mathcal{C}_{\mathcal{A}}^{+}(\sigma) \backslash \mathcal{A}\right) \mid \exists\left(\mathcal{C}_{1}, \ldots, \mathcal{C}_{n}\right) \in J_{\mathcal{C}_{\mathcal{A}}(\sigma), \mathcal{A}}\right.$, and $\exists j \in\{1, \ldots, n\}: \mathcal{C}_{j}=$ $\mathcal{C}$ \}, tube of typical paths from $\sigma$ to $\mathcal{A}$

$\mathcal{U}_{\sigma^{\prime}}(\sigma) \quad\left\{\eta \in \mathcal{X} \mid \exists \omega \in \Omega_{\sigma, \sigma^{\prime}}^{\mathrm{vtj}}\right.$ s.t. $\omega \cap \mathcal{X}^{s} \backslash\left\{\sigma, \sigma^{\prime}\right\}=\varnothing$ and $\left.\eta \in \omega\right\}$, restricted-tube of typical paths from $\sigma$ to $\sigma^{\prime}$

$\mathscr{U}_{\sigma^{\prime}}(\sigma) \quad\left\{\mathcal{C} \in \mathcal{M}\left(\mathcal{C}_{\left\{\sigma^{\prime}\right\}}^{+}(\sigma) \backslash\left\{\sigma^{\prime}\right\}\right) \mid \exists\left(\mathcal{C}_{1}, \ldots, \mathcal{C}_{m}\right) \in J_{\mathcal{C}_{\sigma^{\prime}}(\sigma),\left\{\sigma^{\prime}\right\}}\right.$ s. t. $\bigcup_{i=1}^{m} \mathcal{C}_{i} \cap \mathcal{X}^{s} \backslash\left\{\sigma, \sigma^{\prime}\right\}$ $=\varnothing$ and $\left.\exists j \in\{1, \ldots, n\}: \mathcal{C}_{j}=\mathcal{C}\right\}$, restricted-tube of typical paths from $\sigma$ to $\sigma^{\prime}$

$C^{s}(\sigma) \quad$ Union of all the $s$-cluster in the configuration $\sigma$ for some $s \in S$

$R\left(C^{s}(\sigma)\right) \quad$ Smallest rectangle surrounding $C^{s}(\sigma)$

$R_{\ell_{1} \times \ell_{2}} \quad$ Rectangle in $\mathbb{R}^{2}$ with sides of length $\ell_{1}$ and $\ell_{2}$

$\bar{R}_{a, b}(r, s) \quad$ Set of configurations with all spins $r$, except those, which are $s$, in a rectangle $a \times b$

$\tilde{R}_{a, b}(r, s) \quad$ Set of configurations with all spins $s$, except those, which are $r$, in a rectangle $a \times b$

$\bar{B}_{a, b}^{l}(r, s) \quad$ Set of configurations with all spins $r$, except those, which are $s$, in a rectangle $a \times b$ with a bar $1 \times l$ adjacent to one of the sides of length $b$, with $1 \leq l \leq b-1$

$\tilde{B}_{a, b}^{l}(r, s) \quad$ Set of configurations with all spins $s$, except those, which are $r$, in a rectangle $a \times b$ with a bar $1 \times l$ adjacent to one of the sides of length $b$, with $1 \leq l \leq b-1$

$\overline{\mathscr{H}}(\mathbf{r}, \mathbf{s}) \quad \bar{B}_{1, K}^{1}(r, s) \cup \bigcup_{h=2}^{K-2} \bar{B}_{1, K-1}^{h}(r, s)$

$\widetilde{\mathscr{H}}(\mathbf{r}, \mathbf{s}) \quad \tilde{B}_{1, K}^{1}(r, s) \cup \bigcup_{h=2}^{K-2} \tilde{B}_{1, K-1}^{h}(r, s)$

$\overline{\mathcal{Q}}(\mathbf{r}, \mathbf{s}) \quad \bar{R}_{2, K-1}(r, s) \cup \bigcup_{h=2}^{K-2} \bar{B}_{1, K}^{h}(r, s)$ 


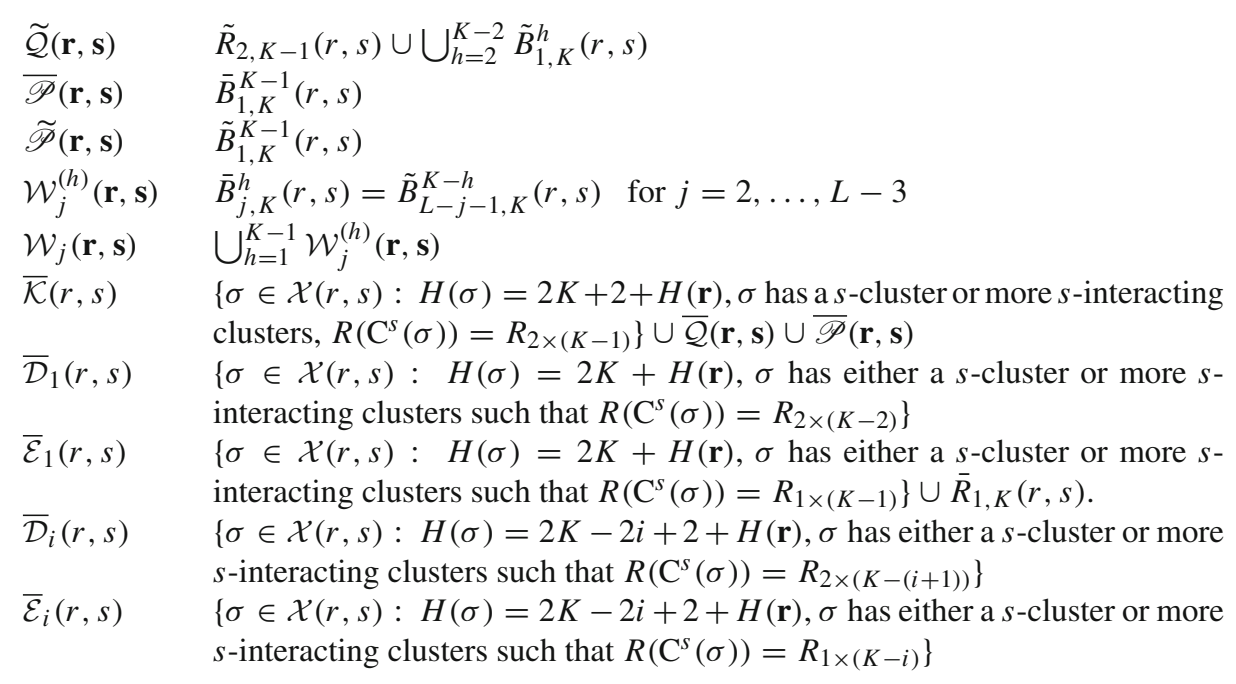

\section{Introduction}

Metastability is a phenomenon that occurs when a physical system is close to a first order phase transition. More precisely, the phenomenon of metastability occurs when a system is trapped for a long time in a state different from the stable state, the so-called metastable state. After a long (random) time or due to random fluctuations the system makes a sudden transition from the metastable state to the stable state. When this happens, the system is said to display metastable behavior. On the other hand, when the system lies on the phase coexistence line, it is of interest to investigate its tunneling behavior, i.e., how the system transitions between the two (or more) stable states. Since metastability occurs in several physical situations, such as supercooled liquids and supersaturated gases, many models for metastable behavior have been formulated throughout the years. Tipically, the evolution of the physical system is approximated by a stochastic process, and broadly speaking the following three main issues are investigated. The first is the study of the first hitting time at which the process starting from a metastable state visits a stable state. The second issue is the study of the so-called set of critical configurations, i.e., the set of those configurations that are crossed by the process during the transition from the metastable state to the stable state. The final issue is the study of the typical trajectories that the system follows during the transition from the metastable state to the stable state. This is the so-called tube of typical paths. The same three issues are investigated when a system displays tunneling behavior, except that in this case one is interested in the transition from one stable state to another stable state.

In this paper we study the tunneling behavior of the $q$-state Potts model on a twodimensional discrete torus. At each site $i$ of the lattice lies a spin with value $\sigma(i) \in\{1, \ldots, q\}$. To each spin configuration we associate an energy such that configurations where neighboring spins have the same value are energetically favored. A model that satisfies this condition is said to be ferromagnetic. The $q$-state Potts model is an extension of the classical Ising model from $q=2$ to an arbitrary number of spins states. We study the $q$-state ferromagnetic Potts model with zero external magnetic field $(h=0)$ in the limit of large inverse temperature $\beta \rightarrow \infty$. When $h=0$, the energy only depends on the local interactions between nearest-neighbor spins. Moreover, when $\beta \gg 1$ there are $q$ stable states, corresponding to 
Fig. 1 Example of configurations belonging to the set of the minimal-restricted gates between the stable configurations $\mathbf{r}$ and $\mathbf{s}$. We color white the vertices with spin $r$, gray those vertices with spin $s$
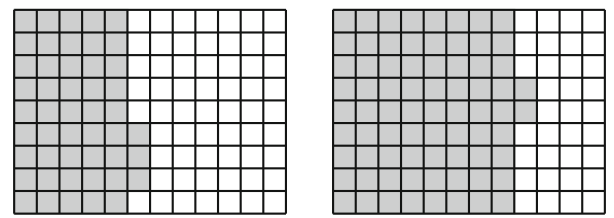

the configurations where all spins are equal. In other words, the system lies on a coexistence line. The stochastic evolution is described by a Glauber-type dynamics, that consists of a single-spin flip Markov chain on a finite state space $\mathcal{X}$ with transition probabilities given by the Metropolis algorithm and with stationary distribution given by the Gibbs measure $\mu_{\beta}$, see (2.7). In this setting, the metastable states are not interesting since they do not have a clear physical interpretation, hence we focus our attention on the tunneling behavior between stable configurations.

The goal of this paper is to investigate the second and third issues introduced above for the tunneling behavior of the system. We describe the set of minimal gates, which have the physical meaning of "critical configurations", and the tube of typical paths for three different types of transitions. More precisely, we study the transition from any stable configuration $\mathbf{r}$ (a) to some other stable configuration $\mathbf{s} \neq \mathbf{r}$ under the constraint that the path followed does not intersect other stable configurations, (b) to any other stable configuration, and (c) to some other stable configuration $\mathbf{s} \neq \mathbf{r}$. In Sect. 3.1.1 (resp. Sect. 4.1.1) we introduce the notion of minimal restricted-gates (resp. restricted-tube of typical paths) to denote the minimal gates (resp. tube of typical paths) for the transition (a). Let us now briefly describe our approach. First we study the energy landscape between two stable configurations. Roughly speaking, we prove that the set of minimal-restricted gates for any transition (a) contains those configurations in which all the spins are $r$ (respectively $s$ ) except those, which have spins $s$ (respectively $r$ ), in a strip that wraps around the shortest side of the torus and that also has a bar attached to one of the two vertical sides, see Fig. 1. We build on this result to describe the set of minimal gates for the transitions (b) and (c). Next we describe the tube of typical trajectories for the transitions (a), (b) and (c). Once again we first describe the restricted-tube of typical paths between two stable configurations, and then we lean on this result to describe the tube of typical paths for the transitions (b) and (c). We show that the restricted-tube of typical paths between the stable states $\mathbf{r}$ and $\mathbf{s}$ includes the minimal-restricted gates, as well as configurations with one or more clusters of spin $s$ (respectively $r$ ) —of at most a certain size, which we identify_-surrounded by spins $r$ (respectively $s$ ). See Sect. 4.1.2 and Figs. 13-15 below. As a special case of our general results we retrieve the minimal gates and the tube of typical paths for the Ising model with zero external magnetic field. We give these respectively at the end of Sects. 3 and of 4 .

Related work. Our work concludes the study of the metastability of the Potts model in the low-temperature regime first initiated in [1], where the authors derive the asymptotic behavior of the first hitting time associated with the transitions (b) and (c) above. They obtain convergence results in probability, in expectation and in distribution. They also investigate the mixing time, which describes the rate of convergence of the process to its stationary distribution $\mu_{\beta}$. They further show that, as $\beta \rightarrow \infty$, the mixing time grows as $\exp (c \ell \beta)$, where $c>0$ is some constant constant factor and $\ell$ is the smallest side length of $\Lambda$.

In [2] the authors study the $q$-Potts model with zero external magnetic field in two and three dimensions. Their manuscript appeared on ArXiv roughly at the same time as ours. While there are some overlaps between the two papers, the works of the two groups were carried out independently of each other. They find sharp estimates for the tunneling time 
(the so-called prefactor) using the potential-theoretic approach and extending the results in [1]. In the second part of their manuscript, the authors focus on the two-dimensional setting. However, the results and the strategy adopted to prove them are quite different from ours. For this type of transitions, they define the set of the gateway configurations in Definition 8.1 that is a model-dependent set visited with probability tending to one as $\beta \rightarrow \infty$, see [2, Corollary 8.9]. This set is different from the classical model-independent definition of gate and of union of minimal gates, see [3] and following papers. The gates and minimal gates have the physical meaning of critical configurations. In Theorems 3.1, 3.3 and 3.5, we give an explicit and thorough geometric description of the minimal gates and union of all minimal gates for the three transitions (a), (b), (c). Moreover, in Corollaries 3.1, 3.2, 3.3 we prove that these sets are crossed with probability tending to one. On the other hand, [2] does not give a complete geometrical characterization of the set of gateway configurations and this set is not a minimal gate. The description of the gateway configurations is suitable to allow them to compute the prefactor. Finally, in our paper we analyse the third issue of metastability by indentifying precisely the tube of typical trajectories. This analysis is absent in [2].

In [4], the authors consider the q-Potts model with non-zero external field and analyze separately the case of positive and negative external magnetic field. In the first scenario there are $q-1$ stable configurations and a unique metastable state. In the second scenario there are $q-1$ degenerate-metastable configurations and only one global minimum. In both cases the authors describe the asymptotic behavior of the first hitting time from the metastable to the stable state as $\beta \rightarrow \infty$, the mixing time, the spectral gap and they identify geometrically the set of gates for these transitions.

We adopt the statistical mechanics framework known as pathwise approach. This is a set of techniques that rely on a detailed knowledge of the energy landscape and on ad hoc large deviations estimates to give a quantitative answer to the three issues of metastability which we described above. The pathwise approach was first introduced in 1984 [5] and then developed in [6-9]. We adopt the convention of listing citations in order of publication date. This approach was further expanded in [3,10-14] to distinguish the study of the transition time and of the gates from the study of typical paths. In $[3,8]$ the pathwise approach was expanded and refined with the aim of finding answers valid with maximal generality and of reducing as much as possible the number of model dependent inputs necessary to study the metastable behaviour of a system.

The pathwise approach was applied in [8,15-21] to study the metastable behaviour of Ising-like models with Glauber dynamics. Moreover, the approach was used in [12,22-26] to find the transition time and the gates for Ising-like and hard-core models with Kawasaki and Glauber dynamics. The pathwise approach was also applied to study probabilistic cellular automata (parallel dynamics) in [27-31].

On the other hand, the so-called potential-theoretical approach exploits a suitable Dirichlet form and spectral properties of the transition matrix to study the hitting time of metastable dynamics. One of the advantages of this approach is that it makes possible the estimation of the expected value of the transition time up to the (lower-order) coefficient that multiplies the (leading-order) exponential term. The coefficient is known in the literature as the pre-factor. These results are grounded in a detailed knowledge of the critical configurations and on the configurations connected to them in one step of the the dynamics, see [32-35]. This method was applied to find the pre-factor for Ising-like models and the hard-core model in [35-41] for Glauber and Kawasaki dynamics and in [42,43] for parallel dynamics. Recently, other approaches have been developed in [44-46] and in [47]. These approaches are particularly well-suited to find the pre-factor when dealing with the tunnelling between two or more stable states. 
The outline of the paper is as follows. At the beginning of Sect. 2, we define the model. In Sect. 3 we give a list of definitions that are necessary in order to state our main results on the set of minimal gates. In Sect. 3.2.1 we give the main results for the minimal restricted-gates for the transition (a). In Sects. 3.2.2 and 3.2.3 we state our main results for the minimal gates for the transitions (b) and (c), respectively. In Sect. 4 we give some additional definitions that are necessary in order to state our results on the tube of typical paths. In Sect. 4.2.1 we state the main results on the restricted-tube of typical paths. In Sect. 4.2.2 and 4.2.3 we give the main results on the tube of typical paths for the transitions (b) and (c), respectively. In Sect. 5 we prove some useful lemmas that allow us to complete the proof of the main results stated in Sect. 3.2.1. In Sect. 6 we carry out the proof of the main results introduced in Sects. 3.2.2-3.2.3. Finally, in Sect. 7 we prove the results on the tube of typical paths between two stable states.

\section{Model Description}

In the $q$-state Potts model each spin lies on the vertices of a finite two-dimensional rectangular lattice $\Lambda=(V, E)$, where $V=\{0, \ldots, K-1\} \times\{0, \ldots, L-1\}$ is the vertex set and $E \subseteq V \times V$ is the set of nearest-neighbors vertices. Without loss of generality, we assume $K \leq L \leq 3$. We consider periodic boundary conditions. Formally, the vertices lying on opposite sides of the rectangle are identified, so that we end up with a two-dimensional torus. To each vertex $v \in V$ is associated a spin value $\sigma(v) \in S:=\{1, \ldots, q\}$. Therefore, a spin configuration $\sigma$ is an element of the set $\mathcal{X}:=S^{V}$. To each configuration $\sigma \in \mathcal{X}$ is associated the energy function (or Hamiltonian)

$$
H(\sigma):=-J_{c} \sum_{(v, w) \in E} \mathbb{1}_{\{\sigma(v)=\sigma(w)\}}, \quad \sigma \in \mathcal{X},
$$

where $J_{c}$ is known as coupling or interaction constant. For our model, $H$ is just the sum of the interaction energy between nearest-neighbor spins. The Potts model is said to be ferromagnetic when $J_{c}>0$, and antiferromagnetic otherwise. In this paper we focus on the ferromagnetic Potts model, and we set $J_{c}=1$ without loss of generality. We denote by $\mathcal{X}^{s}$ the set of the global minima of the Hamiltonian (2.1) in $\mathcal{X}$.

The system evolves according to a Glauber-type dynamics which depends on the the inverse temperature parameter $\beta>0$. Formally, the dynamics is a single-spin update Markov chain $\left\{X_{t}^{\beta}\right\}_{t \in \mathbb{N}}$ on the state space $\mathcal{X}$ with the transition probabilities

$$
P_{\beta}\left(\sigma, \sigma^{\prime}\right):= \begin{cases}Q\left(\sigma, \sigma^{\prime}\right) e^{-\beta\left[H\left(\sigma^{\prime}\right)-H(\sigma)\right]^{+},}, & \text {if } \sigma \neq \sigma^{\prime}, \\ 1-\sum_{\eta \neq \sigma} P_{\beta}(\sigma, \eta), & \text { if } \sigma=\sigma^{\prime},\end{cases}
$$

where $[n]^{+}:=\max \{0, n\}$ is the positive part of $n$ and $Q$ is the connectivity matrix

$$
Q\left(\sigma, \sigma^{\prime}\right):= \begin{cases}\frac{1}{q|V|}, & \text { if }\left|\left\{v \in V: \sigma(v) \neq \sigma^{\prime}(v)\right\}\right|=1, \\ 0, & \text { if }\left|\left\{v \in V: \sigma(v) \neq \sigma^{\prime}(v)\right\}\right|>1 .\end{cases}
$$

We say that $\sigma \in \mathcal{X}$ communicates with $\sigma^{\prime} \in \mathcal{X}$ when $Q\left(\sigma, \sigma^{\prime}\right) \neq 0$. The matrix $Q$ is symmetric and irreducible. This dynamics may be equivalently generated as follows. Given a configuration $\sigma \in \mathcal{X}$, at each step

1. a vertex $v \in V$ and a spin value $s \in S$ are selected independently and uniformly at random; 
2. the spin $\sigma(v)$ at $v$ is updated to spin $s$ with probability

$$
\begin{cases}1, & \text { if } H\left(\sigma^{v, s}\right)-H(\sigma) \leq 0, \\ e^{-\beta\left[H\left(\sigma^{v, s}\right)-H(\sigma)\right]}, & \text { if } H\left(\sigma^{v, s}\right)-H(\sigma)>0,\end{cases}
$$

where $\sigma^{v, s}$ is the configuration obtained from $\sigma$ by updating $\sigma(v)$ to $s$, i.e.,

$$
\sigma^{v, s}(w):= \begin{cases}\sigma(w) & \text { if } w \neq v \\ s & \text { if } w=v .\end{cases}
$$

Hence, at each step the update probability of the selected vertex $v$ depends on the energy difference

$$
H\left(\sigma^{v, s}\right)-H(\sigma)=\sum_{(v, w)}\left(\mathbb{1}_{\{\sigma(v)=\sigma(w)\}}-\mathbb{1}_{\{\sigma(w)=s\}}\right)
$$

It follows from standard results that the dynamics (2.2) is reversible with respect to the so-called Gibbs measure

$$
\mu_{\beta}(\sigma):=\frac{e^{-\beta H(\sigma)}}{\sum_{\sigma^{\prime} \in \mathcal{X}} e^{-\beta H\left(\sigma^{\prime}\right)}} .
$$

In the low-temperature regime $\beta \gg 1, \mu_{\beta}$ is concentrated on the the global minima of $H$. In our setting, we denote by $\mathbf{1}, \ldots, \mathbf{q} \in \mathcal{X}$ the configurations with constant spin values. For example, $\mathbf{1}(v)=1$ for any $v \in V$. By simple algebraic calculations the following proposition is verified.

Proposition 2.1 (Identification of the stable configurations) The set of the global minima of the Hamiltonian (2.1) is given by

$$
\mathcal{X}^{s}:=\{\boldsymbol{1}, \ldots, \boldsymbol{q}\}
$$

Finally, we refer to the triplet $(\mathcal{X}, H, Q)$ as the energy landscape. Note that $\mu_{\beta}$ and $P_{\beta}$ are completely specified by the energy landscape.

\section{Minimal Restricted-Gates and Minimal Gates: Main Results}

In this section we state formally our results on the set of minimal restricted-gates and the one of minimal gates for the transition either from a stable configuration to the other stable states or from a stable state to another stable configuration. However, first we give some notations and definitions which are used throughout the next sections.

\subsection{Definitions and Notations}

Each $v \in V$ is identified by its coordinates $(i, j)$, where $i$ and $j$ denote respectively the row and the column of $\Lambda$ where $v$ lies. The $i$-th row (resp. column) of $\Lambda$ is denoted by $r_{i}$ (resp. $c_{i}$ ).

\subsubsection{Model-Independent Definitions and Notations}

We now give a list of model-independent definitions and notations that will be useful in formulating our main results. 
- A path $\omega$ is a finite sequence of configurations $\omega_{0}, \ldots, \omega_{n} \in \mathcal{X}$ such that $Q\left(\omega_{i}, \omega_{i+1}\right)>$ 0 for $i=0, \ldots, n-1$. A path from $\omega_{0}=\sigma$ to $\omega_{n}=\sigma^{\prime}$ is denoted as $\omega: \sigma \rightarrow \sigma^{\prime}$. Finally, $\Omega_{\sigma, \sigma^{\prime}}$ denotes the set of all paths between $\sigma$ and $\sigma^{\prime}$.

- The height of a path $\omega$ is

$$
\Phi_{\omega}:=\max _{i=0, \ldots, n} H\left(\omega_{i}\right) .
$$

- For any pair $\sigma, \sigma^{\prime} \in \mathcal{X}$, the communication height $\Phi\left(\sigma, \sigma^{\prime}\right)$ between $\sigma$ and $\sigma^{\prime}$ is the minimal energy across all paths $\omega: \sigma \rightarrow \sigma^{\prime}$. Formally,

$$
\Phi\left(\sigma, \sigma^{\prime}\right):=\min _{\omega: \sigma \rightarrow \sigma^{\prime}} \Phi_{\omega}=\min _{\omega: \sigma \rightarrow \sigma^{\prime}} \max _{\eta \in \omega} H(\eta) .
$$

More generally, the communication energy between any pair of non-empty disjoint subsets $\mathcal{A}, \mathcal{B} \subset \mathcal{X}$ is $\Phi(\mathcal{A}, \mathcal{B}):=\min _{\sigma \in \mathcal{A}, \sigma^{\prime} \in \mathcal{B}} \Phi\left(\sigma, \sigma^{\prime}\right)$.

- The set of optimal paths between $\sigma, \sigma^{\prime} \in \mathcal{X}$ is defined as

$$
\Omega_{\sigma, \sigma^{\prime}}^{o p t}:=\left\{\omega \in \Omega_{\sigma, \sigma^{\prime}}: \max _{\eta \in \omega} H(\eta)=\Phi\left(\sigma, \sigma^{\prime}\right)\right\} .
$$

In other words, the optimal paths are those that realize the min-max in (3.2) between $\sigma$ and $\sigma^{\prime}$.

- The set of minimal saddles between $\sigma, \sigma^{\prime} \in \mathcal{X}$ is defined as

$$
\mathcal{S}\left(\sigma, \sigma^{\prime}\right):=\left\{\xi \in \mathcal{X}: \exists \omega \in \Omega_{\sigma, \sigma^{\prime}}^{o p t}, \xi \in \omega: \max _{\eta \in \omega} H(\eta)=H(\xi)\right\} .
$$

- We say that $\eta \in \mathcal{S}\left(\sigma, \sigma^{\prime}\right)$ is an essential saddle if either

- there exists $\omega \in \Omega_{\sigma, \sigma^{\prime}}^{o p t}$ such that $\left\{\operatorname{argmax}_{\omega} H\right\}=\{\eta\}$ or

- there exists $\omega \in \Omega_{\sigma, \sigma^{\prime}}^{\text {opt }}$ such that $\left\{\operatorname{argmax}_{\omega} H\right\} \supset\{\eta\}$ and $\left\{\operatorname{argmax}_{\omega^{\prime}} H\right\} \nsubseteq$ $\left\{\operatorname{argmax}_{\omega} H\right\} \backslash\{\eta\}$ for all $\omega^{\prime} \in \Omega_{\sigma, \sigma^{\prime}}^{o p t}$.

A saddle $\eta \in \mathcal{S}\left(\sigma, \sigma^{\prime}\right)$ that is not essential is said to be unessential.

- Given $\sigma, \sigma^{\prime} \in \mathcal{X}$, we say that $\mathcal{W}\left(\sigma, \sigma^{\prime}\right)$ is a gate for the transition from $\sigma$ to $\sigma^{\prime}$ if $\mathcal{W}\left(\sigma, \sigma^{\prime}\right) \subseteq \mathcal{S}\left(\sigma, \sigma^{\prime}\right)$ and $\omega \cap \mathcal{W}\left(\sigma, \sigma^{\prime}\right) \neq \varnothing$ for all $\omega \in \Omega_{\sigma, \sigma^{\prime}}^{o p t}$

We say that $\mathcal{W}\left(\sigma, \sigma^{\prime}\right)$ is a minimal gate for the transition from $\sigma$ to $\sigma^{\prime}$ if it is a minimal (by inclusion) subset of $\mathcal{S}\left(\sigma, \sigma^{\prime}\right)$ that is visited by all optimal paths, namely, it is a gate and for any $\mathcal{W}^{\prime} \subset \mathcal{W}\left(\sigma, \sigma^{\prime}\right)$ there exists $\omega^{\prime} \in \Omega_{\sigma, \sigma^{\prime}}^{o p t}$ such that $\omega^{\prime} \cap \mathcal{W}^{\prime}=\varnothing$. We denote by $\mathcal{G}=\mathcal{G}\left(\sigma, \sigma^{\prime}\right)$ the union of all minimal gates for the transition from $\sigma$ to $\sigma^{\prime}$.

- Let $\sigma, \sigma^{\prime} \in \mathcal{X}^{s}, \sigma \neq \sigma^{\prime}$, we define restricted-gate for the transition from $\sigma$ to $\sigma^{\prime}$ a subset $\mathcal{W}_{\mathrm{RES}}\left(\sigma, \sigma^{\prime}\right) \subset \mathcal{S}\left(\sigma, \sigma^{\prime}\right)$ which is intersected by all $\omega \in \Omega_{\sigma, \sigma^{\prime}}^{o p t}$ such that $\omega \cap \mathcal{X}^{s} \backslash\left\{\sigma, \sigma^{\prime}\right\}=$ $\varnothing$.

We say that a restricted-gate $\mathcal{W}_{\mathrm{RES}}\left(\sigma, \sigma^{\prime}\right)$ for the transition from $\sigma$ to $\sigma^{\prime}$ is a minimal restricted-gate if for any $\mathcal{W}^{\prime} \subset \mathcal{W}_{\mathrm{RES}}\left(\sigma, \sigma^{\prime}\right)$ there exists $\omega^{\prime} \in \Omega_{\sigma, \sigma^{\prime}}^{\text {opt }}$ such that $\omega^{\prime} \cap \mathcal{W}^{\prime}=$ $\varnothing$. We denote by $\mathcal{F}\left(\sigma, \sigma^{\prime}\right)$ the union of all minimal restricted-gates for the transition from $\sigma$ to $\sigma^{\prime}$. Note that all gates are restricted gates in the case of the Ising model with zero external magnetic field, for which $q=2$, see Corollary 3.4 .

\subsubsection{Model-Dependent Definitions and Notations}

Next we give some further model-dependent notations in order to be able to state our main results. The definitions hold for any $q$-Potts configuration $\sigma \in \mathcal{X}$ and any two different spin values $r, s \in\{1, \ldots, q\}$. 


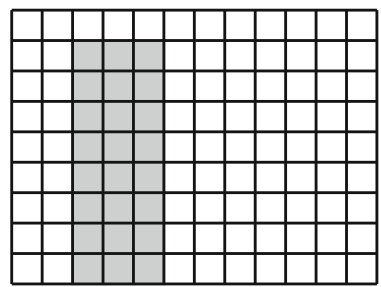

(a)

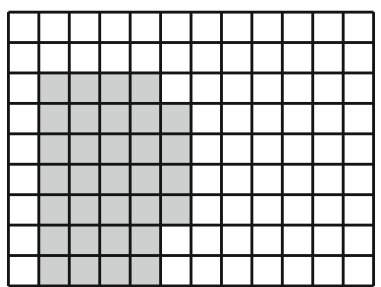

(b)

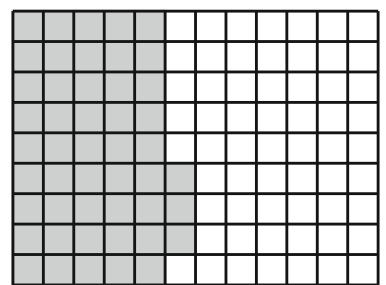

(c)

Fig. 2 Examples of configurations which belong to $\bar{R}_{3,8}(r, s)(\mathbf{a}), \bar{B}_{4,7}^{4}(r, s)(\mathbf{b})$ and $\tilde{\mathcal{B}}_{6,9}^{6}(r, s)(\mathbf{c})$. For semplicity we color white the vertices whose spin is $r$ and we color gray the vertices whose spin is $s$

Fig. 3 Example of configuations belonging to $\overline{\mathscr{P}}(\mathbf{r}, \mathbf{s})$ and $\overline{\mathcal{Q}}(\mathbf{r}, \mathbf{s})$ on a $9 \times 12$ grid $\Lambda$. Gray vertices have spin value $s$, white vertices have spin value $r$. By flipping to $r$ a spin $s$ among those with the lines, the path enters into $\bar{B}_{1, K}^{K-2}(r, s) \subset \overline{\mathcal{Q}}(\mathbf{r}, \mathbf{s})$; instead, by flipping to $r$ a spin $s$ among those with dots, the path goes to $\bar{R}_{2, K-1}(r, s) \subset \overline{\mathcal{Q}}(\mathbf{r}, \mathbf{s})$

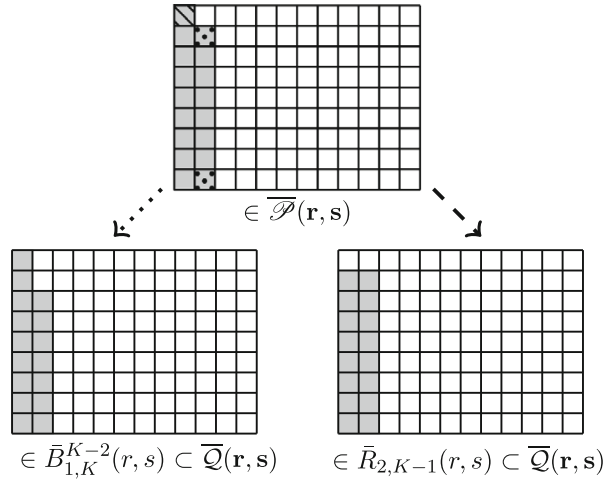

- $\bar{R}_{a, b}(r, s)$ denotes the set of those configurations in which all the vertices have spins equal to $r$, except those, which have spins $s$, in a rectangle $a \times b$, see Fig. 2a;

- $\bar{B}_{a, b}^{h}(r, s)$ denotes the set of those configurations in which all the vertices have spins $r$, except those, which have spins $s$, in a rectangle $a \times b$ with a bar $1 \times h$ adjacent to one of the sides of length $b$, with $1 \leq h \leq b-1$, see Fig. $2 \mathrm{~b}$.

- Analogously, we set $\tilde{R}_{a, b}(r, s)$ and $\tilde{\tilde{B}}_{a, b}^{h}(r, s)$ interchanging the role of spins $r$ and $s$, see Fig. 2c.

Note that

$$
\bar{R}_{a, K}(r, s) \equiv \tilde{R}_{L-a, K}(r, s) \text { and } \bar{B}_{a, K}^{h}(r, s) \equiv \tilde{B}_{L-a-1, K}^{K-h}(r, s) .
$$

Next, we define sets of configurations that are crucial to describe the gate. We show their location on the energy landscape in Fig. 5 below.

- We set

$$
\overline{\mathscr{P}}(\mathbf{r}, \mathbf{s}):=\bar{B}_{1, K}^{K-1}(r, s), \quad \tilde{\mathscr{P}}(\mathbf{r}, \mathbf{s}):=\tilde{B}_{1, K}^{K-1}(r, s) .
$$

We refer to Fig. 3 for an example of a configuration belonging to $\overline{\mathscr{P}}(\mathbf{r}, \mathbf{s})$.

- We define

$$
\overline{\mathcal{Q}}(\mathbf{r}, \mathbf{s}):=\bar{R}_{2, K-1}(r, s) \cup \bigcup_{h=2}^{K-2} \bar{B}_{1, K}^{h}(r, s), \quad \widetilde{\mathcal{Q}}(\mathbf{r}, \mathbf{s}):=\tilde{R}_{2, K-1}(r, s) \cup \bigcup_{h=2}^{K-2} \tilde{B}_{1, K}^{h}(r, s) .
$$


Fig. 4 Example of configuations belonging to $\overline{\mathcal{Q}}(\mathbf{r}, \mathbf{s})$ and $\overline{\mathscr{H}}(\mathbf{r}, \mathbf{s})$ on a $9 \times 12$ grid $\Lambda$. White vertices have spin $r$, gray vertices have spin $s$. By flipping a spin $s$ to $r$ among those with dots, the optimal path remains in $\overline{\mathcal{Q}}(\mathbf{r}, \mathbf{s})$. Otherwise, if a spin $s$ with lines becomes $r$, the path arrives for the first time in $\overline{\mathscr{H}}(\mathbf{r}, \mathbf{s})$. Starting from $\bar{B}_{1, K-1}^{K-2}(r, s)$, the path can pass to another configuration belonging to $\overline{\mathscr{H}}(\mathbf{r}, \mathbf{s})$

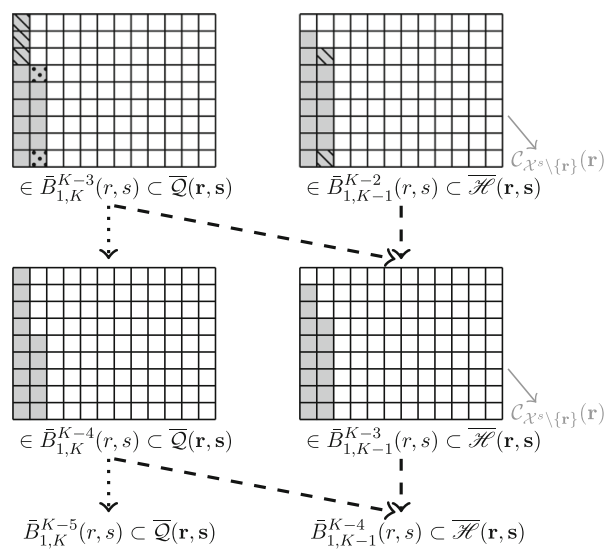

- We define

$$
\overline{\mathscr{H}}(\mathbf{r}, \mathbf{s}):=\bar{B}_{1, K}^{1}(r, s) \cup \bigcup_{h=2}^{K-2} \bar{B}_{1, K-1}^{h}(r, s), \quad \widetilde{\mathscr{H}}(\mathbf{r}, \mathbf{s}):=\tilde{B}_{1, K}^{1}(r, s) \cup \bigcup_{h=2}^{K-2} \tilde{B}_{1, K-1}^{h}(r, s) .
$$

We refer to Figs. 3 and 4 for an example of a configurations belonging to $\overline{\mathcal{Q}}(\mathbf{r}, \mathbf{s})$ and to $\overline{\mathscr{H}}(\mathbf{r}, \mathbf{s})$. See Figs. 9 and 10 for other examples of this type of configurations.

- Finally, we set

$$
\begin{aligned}
\mathcal{W}_{j}^{(h)}(\mathbf{r}, \mathbf{s}) & :=\bar{B}_{j, K}^{h}(r, s)=\tilde{B}_{L-j-1, K}^{K-h}(r, s) \text { for } j=2, \ldots, L-3, \\
\mathcal{W}_{j}(\mathbf{r}, \mathbf{s}) & :=\bigcup_{h=1}^{K-1} \mathcal{W}_{j}^{(h)}(\mathbf{r}, \mathbf{s}) .
\end{aligned}
$$

We refer to Fig. 2c for an example of configuration belonging to $\mathcal{W}_{5}^{(3)}(\mathbf{r}, \mathbf{s})$.

\subsection{Main Results}

We are now ready to state the main results of this section.

\subsubsection{Minimal Restricted-Gates Between Two Potts Stable Configurations}

The set of all minimal restricted-gates for the transition between two given stable configurations $\mathbf{r} \neq \mathbf{s}$ is given in the following theorem.

Theorem 3.1 (Minimal-restricted gates) For every $\mathbf{r}, \mathbf{s} \in \mathcal{X}^{s}, \mathbf{s} \neq \mathbf{r}$, the following sets are minimal restricted-gates for the transition $\mathbf{r} \rightarrow \mathbf{s}:$

(a) $\overline{\mathscr{P}}(\mathbf{r}, \mathbf{s})$ and $\widetilde{\mathscr{P}}(\mathbf{r}, \mathbf{s})$;

(b) $\overline{\mathcal{Q}}(\mathbf{r}, \mathbf{s})$ and $\widetilde{\mathcal{Q}}(\mathbf{r}, \mathbf{s})$;

(c) $\overline{\mathscr{H}}(\mathbf{r}, \mathbf{s})$ and $\widetilde{\mathscr{H}}(\mathbf{r}, \mathbf{s})$;

(d) $\mathcal{W}_{j}^{(h)}(\mathbf{r}, \mathbf{s})$ for any $j=2, \ldots, L-3$ and any $h=1, \ldots, K-1$. 


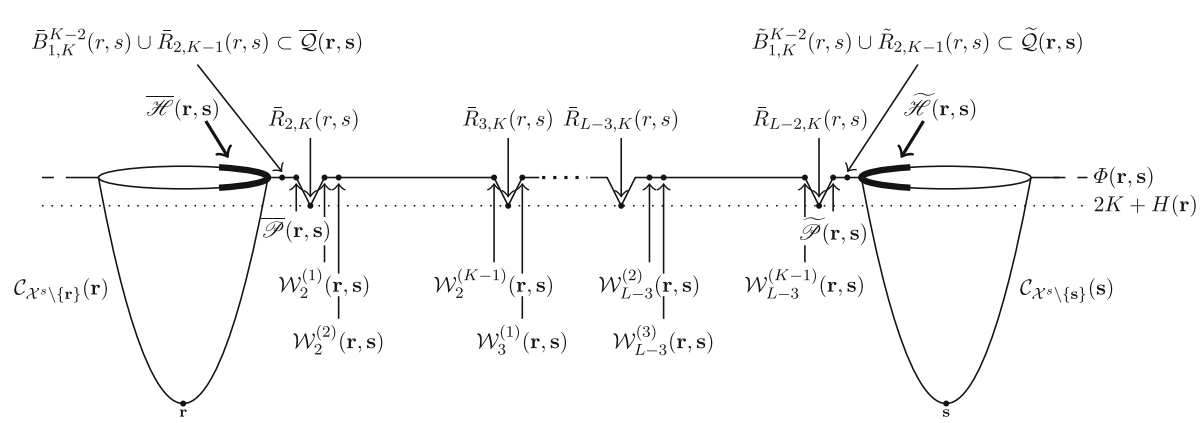

Fig. 5 Focus on the energy landscape between $\mathbf{r}$ and $\mathbf{s}$ and example of some essential saddles for the transition $\mathbf{r} \rightarrow \mathbf{s}$ following an optimal path which does not pass through other stable states

We prove Theorem 3.1 in Sect. 5 by studying the energy landscape between $\mathbf{r}, \mathbf{s}$. In Fig. 5 we give a side view of the energy landscape between two stable configurations $\mathbf{r}$ and $\mathbf{s}$, and we draw the restricted-gates corresponding to the transition between these two configurations. In Fig. 6 we give a top-down view of the energy landscape between several stable configurations. Figure 5 then is a side view of any one of the four arms in Fig. 6. Accordingly, studying the restricted-gates between, say $\mathbf{1}$ and $\mathbf{2}$, corresponds to focusing on only those paths that cross the right part of Fig. 6 . The following results identify the minimal restricted-gates between two stable configurations.

The next theorem implies that there are no other minimal-restricted gates than the ones identified in Theorem 3.1.

Theorem 3.2 (Union of all minimal-restricted gates) For any $\mathbf{r}, \mathbf{s} \in \mathcal{X}^{s}, \mathbf{s} \neq \mathbf{r}$, the union of all minimal restricted-gates for the transition $\mathbf{r} \rightarrow \mathbf{s}$ is given by

$$
\mathcal{F}(\mathbf{r}, \mathbf{s})=\bigcup_{j=2}^{L-3} \mathcal{W}_{j}(\mathbf{r}, \mathbf{s}) \cup \overline{\mathscr{H}}(\mathbf{r}, \mathbf{s}) \cup \widetilde{\mathscr{H}}(\mathbf{r}, \mathbf{s}) \cup \overline{\mathcal{Q}}(\mathbf{r}, \mathbf{s}) \cup \widetilde{\mathcal{Q}}(\mathbf{r}, \mathbf{s}) \cup \overline{\mathscr{P}}(\mathbf{r}, \mathbf{s}) \cup \widetilde{\mathscr{P}}(\mathbf{r}, \mathbf{s}) .
$$

Given a non-empty subset $\mathcal{A} \subset \mathcal{X}$ and a configuration $\sigma \in \mathcal{X}$, we define $\tau_{\mathcal{A}}^{\sigma}:=\inf \{t>0$ : $\left.X_{t}^{\beta} \in \mathcal{A}\right\}$ as the first hitting time of the subset $\mathcal{A}$ for the Markov chain $\left\{X_{t}^{\beta}\right\}_{t \in \mathbb{N}}$ starting from $\sigma$ at time $t=0$.

Corollary 3.1 (Crossing the restricted-gates) Consider any $\mathbf{r}, \mathbf{s} \in \mathcal{X}^{s}$ and the transition from r to $\mathbf{s}$. Then,

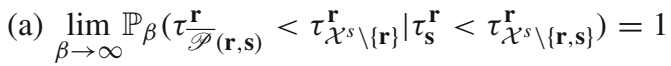
(b) $\lim _{\beta \rightarrow \infty} \mathbb{P}_{\beta}\left(\tau_{\overline{\mathcal{Q}}(\mathbf{r}, \mathbf{s})}^{\mathbf{r}}<\tau_{\mathcal{X}^{s} \backslash\{\mathbf{r}\}}^{\mathbf{r}} \mid \tau_{\mathbf{s}}^{\mathbf{r}}<\tau_{\mathcal{X}^{s} \backslash\{\mathbf{r}, \mathbf{s}\}}^{\mathbf{r}}\right)=1$
(c) $\lim _{\beta \rightarrow \infty} \mathbb{P}_{\beta}\left(\tau \frac{\mathbf{r}}{\mathscr{H}(\mathbf{r}, \mathbf{s})}<\tau_{\mathcal{X}^{s} \backslash\{\mathbf{r}\}}^{\mathbf{r}} \mid \tau_{\mathbf{s}}^{\mathbf{r}}<\tau_{\mathcal{X}^{s} \backslash\{\mathbf{r}, \mathbf{s}\}}^{\mathbf{r}}\right)=1$
(d) $\lim _{\beta \rightarrow \infty} \mathbb{P}_{\beta}\left(\tau_{\mathcal{W}_{j}^{(h)}(\mathbf{r}, \mathbf{s})}^{\mathbf{r}}<\tau_{\mathcal{X}^{s} \backslash\{\mathbf{r}\}}^{\mathbf{r}} \mid \tau_{\mathbf{s}}^{\mathbf{r}}<\tau_{\mathcal{X}^{s} \backslash\{\mathbf{r}, \mathbf{s}\}}^{\mathbf{r}}\right)=1$ for any $j=2, \ldots, L-3$, $h=1, \ldots, K-1$.

Items (a)-(c) hold also for $\widetilde{\mathscr{P}}(\mathbf{r}, \mathbf{s}), \widetilde{\mathcal{Q}}(\mathbf{r}, \mathbf{s}), \widetilde{\mathscr{H}}(\mathbf{r}, \mathbf{s})$, respectively. 


\subsubsection{Minimal Gates for the Transition from a Stable State to the Other Stable States}

Using the results about the minimal restricted-gates, in Theorem 3.3 we identify geometrically all the sets of minimal gates for the transition from a stable configuration to the other stable states. While in Theorem 3.4 we identify the union of all minimal gates for the same transition. We assume $q>2$, otherwise when $q=2,\left|\mathcal{X}^{s}\right|=2$ and Theorems 3.3-3.4 coincide with Theorems 3.1-3.2.

Theorem 3.3 (Minimal gates for the transition $\mathbf{r} \rightarrow \mathcal{X}^{s} \backslash\{\mathbf{r}\}$ ) Let $\mathbf{r} \in \mathcal{X}^{s}$. Then, the following sets are minimal gates for the transition $\mathbf{r} \rightarrow \mathcal{X}^{s} \backslash\{\mathbf{r}\}$ :

(a) $\bigcup_{\mathbf{t} \in \mathcal{X}^{s} \backslash\{\mathbf{r}\}} \overline{\mathscr{P}}(\mathbf{r}, \mathbf{t})$ and $\bigcup_{\mathbf{t} \in \mathcal{X}^{s} \backslash\{\mathbf{r}\}} \tilde{\mathscr{P}}(\mathbf{r}, \mathbf{t})$;

(b) $\bigcup_{\mathbf{t} \in \mathcal{X}^{s} \backslash\{\mathbf{r}\}} \overline{\mathcal{Q}}(\mathbf{r}, \mathbf{t})$ and $\bigcup_{\mathbf{t} \in \mathcal{X}^{s} \backslash\{\mathbf{r}\}} \widetilde{\mathcal{Q}}(\mathbf{r}, \mathbf{t})$;

(c) $\bigcup_{\mathbf{t} \in \mathcal{X}^{s} \backslash\{\mathbf{r}\}} \overline{\mathscr{H}}(\mathbf{r}, \mathbf{t})$ and $\bigcup_{\mathbf{t} \in \mathcal{X}^{s} \backslash\{\mathbf{r}\}} \widetilde{\mathscr{H}}(\mathbf{r}, \mathbf{t})$;

(d) $\bigcup_{\mathbf{t} \in \mathcal{X}^{s} \backslash\{\mathbf{r}\}} \mathcal{W}_{j}^{(h)}(\mathbf{r}, \mathbf{t})$ for any $j=2, \ldots, L-3$ and any $h=1, \ldots, K-1$.

Theorem 3.4 (Union of all minimal gates for the transition $\mathbf{r} \rightarrow \mathcal{X}^{s} \backslash\{\mathbf{r}\}$ ) Given $\mathbf{r} \in \mathcal{X}^{s}$, the union of all minimal gates for the transition $\mathbf{r} \rightarrow \mathcal{X}^{s} \backslash\{\mathbf{r}\}$ is given by

$$
\mathcal{G}\left(\mathbf{r}, \mathcal{X}^{s} \backslash\{\mathbf{r}\}\right)=\bigcup_{\mathbf{t} \in \mathcal{X}^{s} \backslash\{\mathbf{r}\}} \mathcal{F}(\mathbf{r}, \mathbf{t}),
$$

where

$$
\mathcal{F}(\mathbf{r}, \mathbf{t})=\bigcup_{j=2}^{L-3} \mathcal{W}_{j}(\mathbf{r}, \mathbf{t}) \cup \overline{\mathscr{H}}(\mathbf{r}, \mathbf{t}) \cup \widetilde{\mathscr{H}}(\mathbf{r}, \mathbf{t}) \cup \overline{\mathcal{Q}}(\mathbf{r}, \mathbf{t}) \cup \widetilde{\mathcal{Q}}(\mathbf{r}, \mathbf{t}) \cup \overline{\mathscr{P}}(\mathbf{r}, \mathbf{t}) \cup \widetilde{\mathscr{P}}(\mathbf{r}, \mathbf{t}) .
$$

Remark 3.1 Note that when $A=\{r\}$ and $B=S \backslash\{r\}$ the set of model-dependent gateway configurations given in [2, Definition 8.1] contains strictly $\mathcal{G}\left(\mathbf{r}, \mathcal{X}^{s} \backslash\{\mathbf{r}\}\right)$, thus it is a gate but it is not minimal.

We refer to Fig. 5 for an illustration of the energy landscape between two Potts stable states. Moreover, in Fig. 6, we depict an example of restricted-gates for 5-state Potts model in which the set of minimal restricted-gate corresponds to one of the arms that collegues two different stable states.

Corollary 3.2 (Crossing the gates) Consider any $\mathbf{r} \in \mathcal{X}^{s}$ and the transition from $\mathbf{r}$ to $\mathcal{X}^{s} \backslash\{\mathbf{r}\}$. Then, the following properties hold:

$$
\begin{aligned}
& \text { (a) } \lim _{\beta \rightarrow \infty} \mathbb{P}_{\beta}\left(\tau_{\bigcup_{\mathbf{t} \in \mathcal{X}^{s} \backslash\{\mathbf{r}\}}^{\mathbf{r}} \overline{\mathscr{P}}(\mathbf{r}, \mathbf{t})}<\tau_{\mathcal{X}^{s} \backslash\{\mathbf{r}\}}^{\mathbf{r}}\right)=1 \text {; } \\
& \text { (b) } \lim _{\beta \rightarrow \infty} \mathbb{P}_{\beta}\left(\tau_{\bigcup_{\mathbf{t} \in \mathcal{X}^{s} \backslash\{\mathbf{r}\}}^{\mathbf{r}} \overline{\mathcal{Q}}(\mathbf{r}, \mathbf{t})}<\tau_{\mathcal{X}^{s} \backslash\{\mathbf{r}\}}^{\mathbf{r}}\right)=1 \text {; }
\end{aligned}
$$

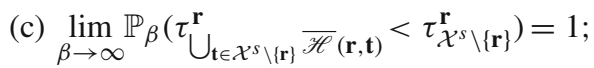

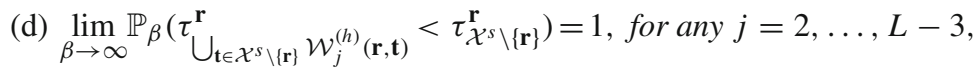

$$
\begin{aligned}
& h=1, \ldots, K-1 \text {. }
\end{aligned}
$$

Items (a)-(c) are also satisfiedfor $\bigcup_{\mathbf{t} \in \mathcal{X}^{s} \backslash\{\mathbf{r}\}} \widetilde{\mathscr{P}}(\mathbf{r}, \mathbf{t}), \bigcup_{\mathbf{t} \in \mathcal{X}^{s} \backslash\{\mathbf{r}\}} \widetilde{\mathcal{Q}}(\mathbf{r}, \mathbf{t})$, and $\bigcup_{\mathbf{t} \in \mathcal{X}^{s} \backslash\{\mathbf{r}\}} \widetilde{\mathscr{H}}(\mathbf{r}, \mathbf{t})$, respectively. Moreover, (a)-(d) imply

$$
\lim _{\beta \rightarrow \infty} \mathbb{P}_{\beta}\left(\tau_{\mathcal{G}\left(\mathbf{r}, \mathcal{X}^{s} \backslash\{\mathbf{r}\}\right)}^{\mathbf{r}}<\tau_{\mathcal{X}^{s} \backslash\{\mathbf{r}\}}^{\mathbf{r}}\right)=1 .
$$




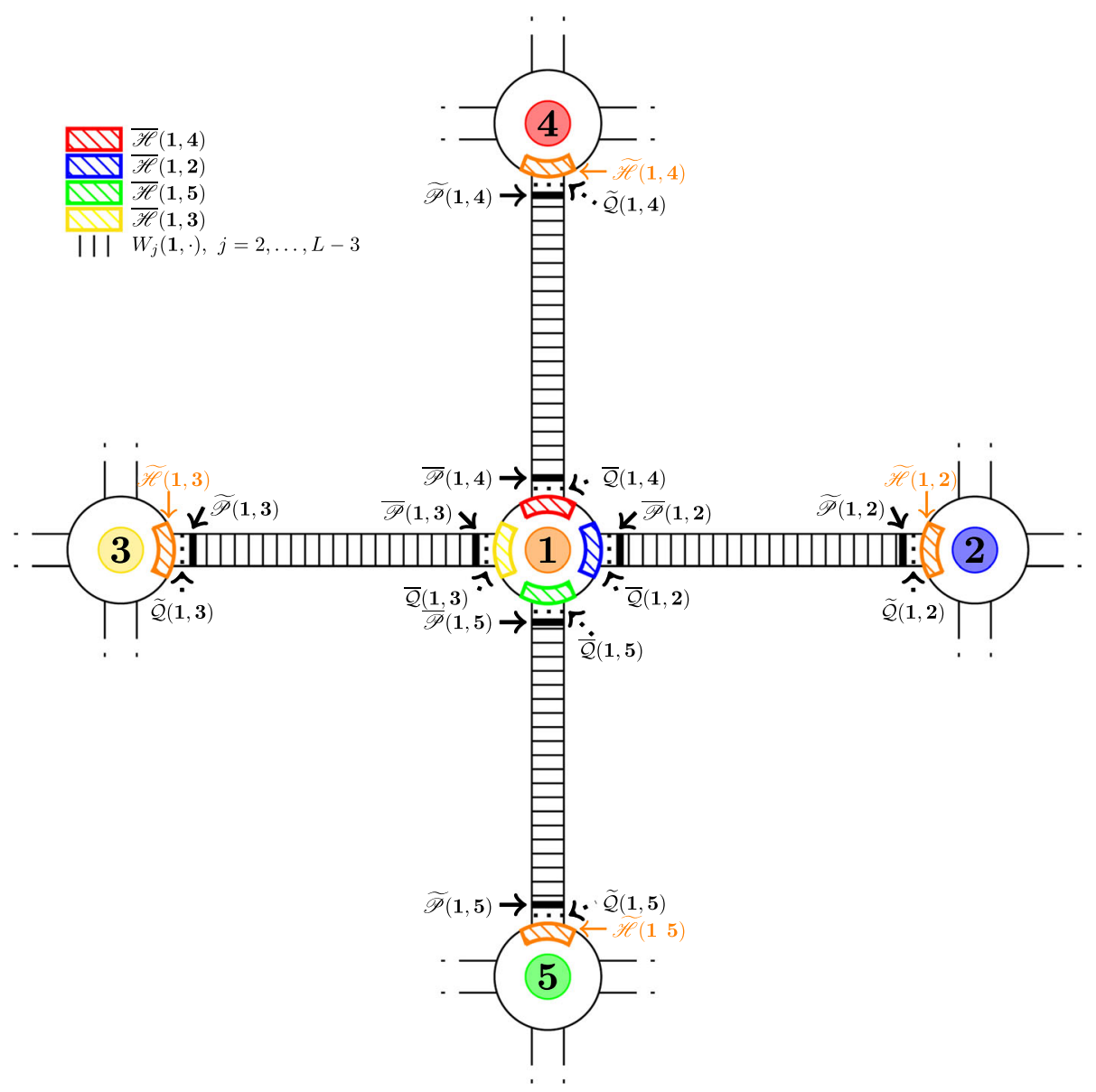

Fig. 6 Example of 5-Potts model with $S=\{1,2,3,4,5\}$. Viewpoint from above on the set of minimal gates around the stable state $\mathbf{1}$ at energy $2 K+2+H(\mathbf{1})$. For any $\mathbf{s} \in\{\mathbf{2}, \mathbf{3}, \mathbf{4}, \mathbf{5}\}$, starting from $\mathbf{1}$, the process hits $\mathcal{X}^{s} \backslash\{\mathbf{1}\}$ for the first time in $\mathbf{s}$ with probability $\frac{1}{q-1}=\frac{1}{4}$

This corollary implies that every geometrical gate and their union have to be crossed with probability tending to one in the asymptotic limit. In [2], the authors prove Corollary 8.9 that is similar to Corollary 3.2(d).

\subsubsection{Minimal Gates for the Transition from a Stable State to Another Stable State}

In Theorem 3.5 we identify geometrically all the sets of minimal gates for the transition from a stable configuration to the another stable state. While in Theorem 3.6 we fully geometrically describe the union of all minimal gates for the same transition. We assume $q>2$, otherwise when $q=2,\left|\mathcal{X}^{s}\right|=2$ and Theorems 3.5-3.6 coincide with Theorems 3.1-3.2. We invite the reader to see Fig. 6 for a pictorial illustration of the structure of the minimal gates. 
Theorem 3.5 (Minimal gates for the transition $\mathbf{r} \rightarrow \mathbf{s}$ ) Consider $\mathbf{r}, \mathbf{s} \in \mathcal{X}^{s}, \mathbf{r} \neq \mathbf{s}$. Then, the following sets are minimal gates for the transition $\mathbf{r} \rightarrow \mathbf{s}$ :

(a) $\bigcup_{\mathbf{t} \in \mathcal{X}^{s} \backslash\{\mathbf{r}\}} \overline{\mathscr{P}}(\mathbf{r}, \mathbf{t}), \bigcup_{\mathbf{t} \in \mathcal{X}^{s} \backslash\{\mathbf{s}\}} \overline{\mathscr{P}}(\mathbf{t}, \mathbf{s}), \bigcup_{\mathbf{t} \in \mathcal{X}^{s} \backslash\{\mathbf{r}\}} \widetilde{\mathscr{P}}(\mathbf{r}, \mathbf{t}), \bigcup_{\mathbf{t} \in \mathcal{X}^{s} \backslash\{\mathbf{s}\}} \tilde{\mathscr{P}}(\mathbf{t}, \mathbf{s})$;

(b) $\bigcup_{\mathbf{t} \in \mathcal{X}^{s} \backslash\{\mathbf{r}\}} \overline{\mathcal{Q}}(\mathbf{r}, \mathbf{t}), \bigcup_{\mathbf{t} \in \mathcal{X}^{s} \backslash\{\mathbf{s}\}} \overline{\mathcal{Q}}(\mathbf{t}, \mathbf{s}), \bigcup_{\mathbf{t} \in \mathcal{X}^{s} \backslash\{\mathbf{r}\}} \widetilde{\mathcal{Q}}(\mathbf{r}, \mathbf{t}), \bigcup_{\mathbf{t} \in \mathcal{X}^{s} \backslash\{\mathbf{s}\}} \widetilde{\mathcal{Q}}(\mathbf{t}, \mathbf{s})$;

(c) $\bigcup_{\mathbf{t} \in \mathcal{X}^{s} \backslash\{\mathbf{r}\}} \overline{\mathscr{H}}(\mathbf{r}, \mathbf{t}), \bigcup_{\mathbf{t} \in \mathcal{X}^{s} \backslash\{\mathbf{s}\}} \overline{\mathscr{H}}(\mathbf{t}, \mathbf{s}), \bigcup_{\mathbf{t} \in \mathcal{X}^{s} \backslash\{\mathbf{r}\}} \widetilde{\mathscr{H}}(\mathbf{r}, \mathbf{t}), \bigcup_{\mathbf{t} \in \mathcal{X}^{s} \backslash\{\mathbf{s}\}} \widetilde{\mathscr{H}}(\mathbf{t}, \mathbf{s})$;

(d) $\bigcup_{\mathbf{t} \in \mathcal{X}^{s} \backslash\{\mathbf{r}\}} \mathcal{W}_{j}^{(h)}(\mathbf{r}, \mathbf{t}), \bigcup_{\mathbf{t} \in \mathcal{X}^{s} \backslash\{\mathbf{s}\}} \mathcal{W}_{j}^{(h)}(\mathbf{t}, \mathbf{s})$ for any $j=2, \ldots, L-3, h=1, \ldots, K-1$.

Theorem 3.6 (Union of all minimal gates for the transition $\mathbf{r} \rightarrow \mathbf{s}$ ) Consider $\mathbf{r}, \mathbf{s} \in \mathcal{X}^{s}$, $\mathbf{r} \neq \mathbf{s}$. Then, the union of all minimal gates for the transition $\mathbf{r} \rightarrow \mathbf{s}$ is given by

$$
\mathcal{G}(\mathbf{r}, \mathbf{s})=\bigcup_{\mathbf{t} \in \mathcal{X}^{s} \backslash\{\mathbf{r}\}} \mathcal{F}(\mathbf{r}, \mathbf{t}) \cup \bigcup_{\mathbf{t} \in \mathcal{X}^{s} \backslash\{\mathbf{s}\}} \mathcal{F}(\mathbf{t}, \mathbf{s}),
$$

where, for any $\mathbf{t}, \mathbf{z} \in \mathcal{X}^{s}, \mathbf{t} \neq \mathbf{z}$,

$$
\mathcal{F}(\mathbf{t}, \mathbf{z})=\bigcup_{j=2}^{L-3} \mathcal{W}_{j}(\mathbf{t}, \mathbf{z}) \cup \overline{\mathscr{H}}(\mathbf{t}, \mathbf{z}) \cup \widetilde{\mathscr{H}}(\mathbf{t}, \mathbf{z}) \cup \overline{\mathcal{Q}}(\mathbf{t}, \mathbf{z}) \cup \widetilde{\mathcal{Q}}(\mathbf{t}, \mathbf{z}) \cup \overline{\mathscr{P}}(\mathbf{t}, \mathbf{z}) \cup \widetilde{\mathscr{P}}(\mathbf{t}, \mathbf{z})
$$

Corollary 3.3 (Crossing the gates) Consider any $\mathbf{r}, \mathbf{s} \in \mathcal{X}^{s}$, with $\mathbf{r} \neq \mathbf{s}$, and the transition from $\mathbf{r}$ to $\mathbf{s}$. Then, the following properties hold:

(a) $\lim _{\beta \rightarrow \infty} \mathbb{P}_{\beta}\left(\tau_{\bigcup_{\left.\mathbf{t} \in \mathcal{X}^{s} \backslash \mathbf{r} \mathbf{r}\right\}}^{\mathbf{r}} \overline{\mathscr{P}}(\mathbf{r}, \mathbf{t})}<\tau_{\mathbf{s}}^{\mathbf{r}}\right)=1, \lim _{\beta \rightarrow \infty} \mathbb{P}_{\beta}\left(\tau_{\bigcup_{\mathbf{t} \in \mathcal{X}^{s} \backslash\{\mathbf{s}\}}^{\mathbf{r}}} \overline{\mathscr{P}}_{(\mathbf{t}, \mathbf{s})}<\tau_{\mathbf{s}}^{\mathbf{r}}\right)=1 ;$

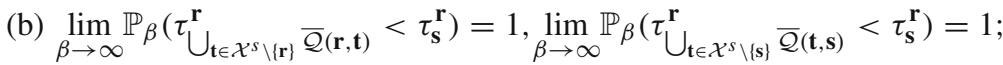

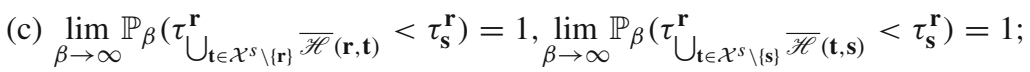

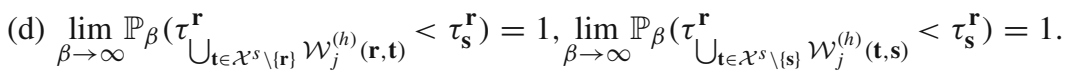

for any $j=2, \ldots, L-3, h=1, \ldots, K-1$. Items (a)-(c) hold also for $\bigcup_{\mathbf{t} \in \mathcal{X}^{s} \backslash\{\mathbf{r}\}} \tilde{\mathscr{P}}(\mathbf{r}, \mathbf{t})$, $\bigcup_{\mathbf{t} \in \mathcal{X}^{s} \backslash\{\mathbf{s}\}} \tilde{\mathscr{P}}(\mathbf{t}, \mathbf{s}), \bigcup_{\mathbf{t} \in \mathcal{X}^{s} \backslash\{\mathbf{r}\}} \widetilde{\mathcal{Q}}(\mathbf{r}, \mathbf{t}), \bigcup_{\mathbf{t} \in \mathcal{X}^{s} \backslash\{\mathbf{s}\}} \widetilde{\mathcal{Q}}(\mathbf{t}, \mathbf{s})$,

$\bigcup_{\mathbf{t} \in \mathcal{X}^{s} \backslash\{\mathbf{r}\}} \widetilde{\mathscr{H}}(\mathbf{r}, \mathbf{t}), \bigcup_{\mathbf{t} \in \mathcal{X}^{s} \backslash\{\mathbf{s}\}} \widetilde{\mathscr{H}}(\mathbf{t}, \mathbf{s})$, respectively. Moreover, (a)-(d) imply

$$
\lim _{\beta \rightarrow \infty} \mathbb{P}_{\beta}\left(\tau_{\mathcal{G}(\mathbf{r}, \mathbf{s})}^{\mathbf{r}}<\tau_{\mathbf{s}}^{\mathbf{r}}\right)=1
$$

This corollary implies that every geometrical gate for the transition $\mathbf{r} \rightarrow \mathbf{s}$ and their union have to be crossed with probability tending to one in the asymptotic limit.

\subsubsection{Minimal Gates of the Ising Model with Zero External Magnetic Field}

When $q=2$, the Potts model corresponds to the Ising model with no external magnetic field, in which $S=\{-1,+1\}$ and $\mathcal{X}^{s}=\{-\mathbf{1},+\mathbf{1}\}$. In this scenario, starting from $\mathbf{- 1}$, the target is necessarily $+\mathbf{1}$ and the following corollary holds. 
Corollary 3.4 Consider the Ising model on a $K \times L$ grid $\Lambda$ with periodic boundary conditions and with zero external magnetic field. Then, the following sets are minimal gates for the transition $-\mathbf{1} \rightarrow+\mathbf{1}$ :

(a) $\overline{\mathscr{P}}(-\mathbf{1},+\mathbf{1}), \tilde{\mathscr{P}}(-\mathbf{1},+\mathbf{1})$;

(b) $\overline{\mathcal{Q}}(-\mathbf{1},+\mathbf{1}), \widetilde{\mathcal{Q}}(-\mathbf{1},+\mathbf{1})$;

(c) $\overline{\mathscr{H}}(-\mathbf{1},+\mathbf{1}), \widetilde{\mathscr{H}}(-\mathbf{1},+\mathbf{1})$;

(d) $\mathcal{W}_{j}^{(h)}(-\mathbf{1},+\mathbf{1})$ for any $j=2, \ldots, L-3$ and any $h=1, \ldots, K-1$.

Moreover

$$
\begin{gathered}
\mathcal{G}(-\mathbf{1},+\mathbf{1})=\bigcup_{j=2}^{L-3} \mathcal{W}_{j}(-\mathbf{1},+\mathbf{1}) \cup \overline{\mathscr{H}}(-\mathbf{1},+\mathbf{1}) \cup \widetilde{\mathscr{H}}(-\mathbf{1},+\mathbf{1}) \cup \overline{\mathcal{Q}}(-\mathbf{1},+\mathbf{1}) \\
\cup \widetilde{\mathcal{Q}}(-\mathbf{1},+\mathbf{1}) \cup \overline{\mathscr{P}}(-\mathbf{1},+\mathbf{1}) \cup \widetilde{\mathscr{P}}(-\mathbf{1},+\mathbf{1}) .
\end{gathered}
$$

\section{Restricted-Tube of Typical Paths and Tube of Typical Paths: Main Results}

In this section we state formally our results on the restricted-tube of typical paths for the transition from one stable state to another. We then state our results on the tube of typical paths for the transition from a stable state to any other stable configuration and for the transition from one stable state to another fixed stable configuration. However, first we give some notations and definitions which are used throughout the next sections.

\subsection{Definitions and Notations}

In order to state our results, we make use of the definitions in Sect. 3.1, as well as some new ones.

\subsubsection{Model-Independent Definitions and Notations}

The following definitions are taken from $[8,11,12]$.

- The bottom $\mathscr{F}(\mathcal{A})$ of a non-empty set $\mathcal{A} \subset \mathcal{X}$ is the set of global minima of $H$ in $\mathcal{A}$, i.e., $\mathscr{F}(\mathcal{A}):=\left\{\eta \in \mathcal{A}: H(\eta)=\min _{\sigma \in \mathcal{A}} H(\sigma)\right\}$.

- A non-empty subset $\mathcal{A} \subseteq \mathcal{X}$ is said to be connected if for any $\sigma, \eta \in \mathcal{A}$ there exists a path $\omega: \sigma \rightarrow \eta$ contained in $\mathcal{A}$. Moreover,

$$
\partial \mathcal{A}:=\{\eta \notin \mathcal{A}: P(\sigma, \eta)>0 \text { for some } \sigma \in \mathcal{A}\} .
$$

is the external boundary of $\mathcal{A}$.

- A connected set of equal energy states which is maximal by inclusion is called a plateau.

- A non-empty subset $\mathcal{C} \subset \mathcal{X}$ is called cycle if it is either a singleton or a connected set such that

$$
\max _{\sigma \in \mathcal{C}} H(\sigma)<H(\mathscr{F}(\partial \mathcal{C})) .
$$

When $\mathcal{C}$ is a singleton, it is said to be a trivial cycle. An extended cycle is a collection of cycles which belong to the same plateau. $\mathscr{C}(\mathcal{X})$ denotes the set of cycles and extended cycles. 
- The principal boundary of $\mathcal{C} \in \mathscr{C}(\mathcal{X})$ is

$$
\mathcal{B}(\mathcal{C}):= \begin{cases}\mathscr{F}(\partial \mathcal{C}) & \text { if } \mathcal{C} \text { is non-trivial cycle, } \\ \{\eta \in \partial \mathcal{C}: H(\eta)<H(\sigma)\} & \text { if } \mathcal{C}=\{\sigma\} \text { is trivial cycle, } \\ \{\eta \in \partial \mathcal{C}: \exists \sigma \in \mathcal{C} \text { s.t. } H(\eta)<H(\sigma)\} & \text { if } \mathcal{C} \text { is extended cycle. }\end{cases}
$$

The non-principal boundary of $\mathcal{C}$ is then $\partial^{n p} \mathcal{C}:=\partial \mathcal{C} \backslash \mathcal{B}(\mathcal{C})$.

- Given a non-empty set $\mathcal{A}$ and $\sigma \in \mathcal{X}$, the initial cycle $\mathcal{C}_{\mathcal{A}}(\sigma)$ is

$$
\mathcal{C}_{\mathcal{A}}(\sigma):=\{\sigma\} \cup\{\eta \in \mathcal{X}: \Phi(\sigma, \eta)<\Phi(\sigma, \mathcal{A})\} .
$$

If $\sigma \in \mathcal{A}$, then $\mathcal{C}_{\mathcal{A}}(\sigma)=\{\sigma\}$ and thus is a trivial cycle. Otherwise, $\mathcal{C}_{\mathcal{A}}(\sigma)$ is either a trivial cycle (when $\Phi(\sigma, \mathcal{A})=H(\sigma)$ ) or a non-trivial cycle containing $\sigma$ (when $\Phi(\sigma, \mathcal{A})>$ $H(\sigma))$. In any case, if $\sigma \notin \mathcal{A}$, then $\mathcal{C}_{\mathcal{A}}(\sigma) \cap \mathcal{A}=\varnothing$.

- The relevant cycle $\mathcal{C}_{\mathcal{A}}^{+}(\sigma)$ is

$$
\mathcal{C}_{\mathcal{A}}^{+}(\sigma):=\{\eta \in \mathcal{X}: \Phi(\sigma, \eta)<\Phi(\sigma, \mathcal{A})+\delta / 2\},
$$

where $\delta$ is the minimum energy gap between an optimal and a non-optimal path from $\sigma$ to $\mathcal{A}$.

- For any non-empty set $\mathcal{A} \subset \mathcal{X}, \mathcal{M}(\mathcal{A})$ denotes the collection of maximal cycles and extended cycles that partitions $\mathcal{A}$. Formally,

$$
\mathcal{M}(\mathcal{A}):=\{\mathcal{C} \in \mathscr{C}(\mathcal{X}) \mid \mathcal{C} \text { maximal by inclusion under constraint } \mathcal{C} \subseteq \mathcal{A}\} .
$$

- A cycle-path is a finite sequence $\left(\mathcal{C}_{1}, \ldots, \mathcal{C}_{m}\right)$ of any combination of trivial, non-trivial and extended cycles $\mathcal{C}_{1}, \ldots, \mathcal{C}_{m} \in \mathscr{C}(\mathcal{X})$, such that $\mathcal{C}_{i} \cap \mathcal{C}_{i+1}=\varnothing$ and $\partial \mathcal{C}_{i} \cap \mathcal{C}_{i+1} \neq \varnothing$, for every $i=1, \ldots, m-1$. The set of cycle-paths that lead from $\sigma$ to $\mathcal{A}$ and consist of maximal cycles in $\mathcal{X} \backslash \mathcal{A}$ is

$$
\mathcal{P}_{\sigma, \mathcal{A}}:=\left\{\left(\mathcal{C}_{1}, \ldots, \mathcal{C}_{m}\right) \mid \mathcal{C}_{1}, \ldots, \mathcal{C}_{m} \in \mathcal{M}\left(\mathcal{C}_{\mathcal{A}}^{+}(\sigma) \backslash A\right), \sigma \in \mathcal{C}_{1}, \partial \mathcal{C}_{m} \cap \mathcal{A} \neq \varnothing\right\} .
$$

- Given a non-empty set $\mathcal{A} \subset \mathcal{X}$ and $\sigma \in \mathcal{X}$, we constructively define a mapping $G$ : $\Omega_{\sigma, A} \rightarrow \mathcal{P}_{\sigma, \mathcal{A}}$. More precisely, given $\omega=\left(\omega_{1}, \ldots, \omega_{n}\right) \in \Omega_{\sigma, \mathcal{A}}$, we set $m_{0}=1, \mathcal{C}_{1}=$ $\mathcal{C}_{\mathcal{A}}(\sigma)$ and define recursively $m_{i}:=\min \left\{k>m_{i-1} \mid \omega_{k} \notin \mathcal{C}_{i}\right\}$ and $\mathcal{C}_{i+1}:=\mathcal{C}_{\mathcal{A}}\left(\omega_{m_{i}}\right)$. We note that $\omega$ is a finite sequence and $\omega_{n} \in \mathcal{A}$, so there exists an index $n(\omega) \in \mathbb{N}$ such that $\omega_{m_{n(\omega)}}=\omega_{n} \in \mathcal{A}$ and there the procedure stops. The way the sequence $\left(\mathcal{C}_{1}, \ldots, \mathcal{C}_{m_{n(\omega)}}\right)$ is constructed shows that it is a cycle-path with $\mathcal{C}_{1}, \ldots, \mathcal{C}_{m_{n(\omega)}} \subset \mathcal{M}(\mathcal{X} \backslash \mathcal{A})$. Moreover, the fact that $\omega \in \Omega_{\sigma, \mathcal{A}}$ implies that $\sigma \in \mathcal{C}_{1}$ and that $\partial \mathcal{C}_{n(\omega)} \cap \mathcal{A} \neq \varnothing$, hence $G(\omega) \in \mathcal{P}_{\sigma, \mathcal{A}}$ and the mapping is well-defined.

- A cycle-path $\left(\mathcal{C}_{1}, \ldots, \mathcal{C}_{m}\right)$ is said to be connected via typical jumps to $\mathcal{A} \subset \mathcal{X}$ or simply $v t j-$ connected to $\mathcal{A}$ if

$$
\mathcal{B}\left(\mathcal{C}_{i}\right) \cap \mathcal{C}_{i+1} \neq \varnothing, \quad \forall i=1, \ldots, m-1, \quad \text { and } \mathcal{B}\left(\mathcal{C}_{m}\right) \cap \mathcal{A} \neq \varnothing .
$$

$J_{\mathcal{C}, \mathcal{A}}$ denotes the collection of all cycle-path $\left(\mathcal{C}, \mathcal{C}_{1}, \ldots, \mathcal{C}_{m}\right)$ that begin in $\mathcal{C}$ and are vtj-connected to $\mathcal{A}$.

- We say that $\omega \in \Omega_{\sigma, \mathcal{A}}$ is a typical path from $\sigma \in \mathcal{X}$ to $\mathcal{A} \subseteq \mathcal{X}$ if its corresponding cyclepath $G(\omega)$ is vtj-connected to $\mathcal{A}$ and we denote by $\Omega_{\sigma, \mathcal{A}}^{\mathrm{vtj}}$ the collection of all typical paths from $\sigma$ to $\mathcal{A}$. Formally,

$$
\Omega_{\sigma, \mathcal{A}}^{\mathrm{vtj}}:=\left\{\omega \in \Omega_{\sigma, \mathcal{A}} \mid G(\omega) \in J_{\mathcal{C}_{\mathcal{A}}(\sigma), \mathcal{A}}\right\} .
$$

See [12, Lemma 3.12] for an equivalent characterization of a typical path. 
- The tube of typical paths $T_{\mathcal{A}}(\sigma)$ from $\sigma$ to $\mathcal{A}$ is the subset of states $\eta \in \mathcal{X}$ that can be reached from $\sigma$ by means of a typical path which does not enter $\mathcal{A}$ before visiting $\eta$. Formally,

$$
T_{\mathcal{A}}(\sigma):=\left\{\eta \in \mathcal{X} \mid \exists \omega \in \Omega_{\sigma, \mathcal{A}}^{\mathrm{vtj}}: \eta \in \omega\right\} .
$$

Moreover, $\mathfrak{T}_{\mathcal{A}}(\sigma)$ is the set of all maximal cycles and maximal extended cycles that belong to at least one vtj-connected path from $\mathcal{C}_{\mathcal{A}}(\sigma)$ to $\mathcal{A}$,

$$
\begin{gathered}
\mathfrak{T}_{\mathcal{A}}(\sigma):=\left\{\mathcal{C} \in \mathcal{M}\left(\mathcal{C}_{\mathcal{A}}^{+}(\sigma) \backslash \mathcal{A}\right) \mid \exists\left(\mathcal{C}_{1}, \ldots, \mathcal{C}_{n}\right) \in J_{\mathcal{C}_{\mathcal{A}}(\sigma), \mathcal{A}},\right. \\
\text { and } \left.\exists j \in\{1, \ldots, n\}: \mathcal{C}_{j}=\mathcal{C}\right\} .
\end{gathered}
$$

Note that $\mathfrak{T}_{\mathcal{A}}(\sigma)=\mathcal{M}\left(T_{\mathcal{A}}(\sigma) \backslash \mathcal{A}\right)$ and that the boundary of $T_{\mathcal{A}}(\sigma)$ consists of states either in $\mathcal{A}$ or in the non-principal part of the boundary of some $\mathcal{C} \in \mathfrak{T}_{\mathcal{A}}(\sigma)$ :

$$
\partial T_{\mathcal{A}}(\sigma) \backslash \mathcal{A} \subseteq \bigcup_{\mathcal{C} \in \mathfrak{T}_{\mathcal{A}}(\sigma)}(\partial \mathcal{C} \backslash \mathcal{B}(\mathcal{C}))=: \partial^{n p} \mathfrak{T}_{\mathcal{A}}(\sigma) .
$$

For the sake of semplicity, we will also refer to $\mathfrak{T}_{\mathcal{A}}(\sigma)$ as tube of typical paths from $\sigma$ to $\mathcal{A}$.

- When $\left|\mathcal{X}^{s}\right|>2$, the restricted-tube of typical paths $\mathcal{U}_{\sigma^{\prime}}(\sigma)$ between two stable states $\sigma$ and $\sigma^{\prime} \neq \sigma$ is the subset of states $\eta \in \mathcal{X}$ that can be reached from $\sigma$ by means of a typical path which does not intersect $\mathcal{X}^{s} \backslash\left\{\sigma, \sigma^{\prime}\right\}$ and does not visit $\sigma^{\prime}$ before visiting $\eta$. Formally,

$$
\mathcal{U}_{\sigma^{\prime}}(\sigma):=\left\{\eta \in \mathcal{X} \mid \exists \omega \in \Omega_{\sigma, \sigma^{\prime}}^{\mathrm{vtj}} \text { s.t. } \omega \cap \mathcal{X}^{s} \backslash\left\{\sigma, \sigma^{\prime}\right\}=\varnothing \text { and } \eta \in \omega\right\} .
$$

Moreover, we set $\mathscr{U}_{\sigma^{\prime}}(\sigma)$ as the set of maximal cycles and maximal extended cycles that belong to at last one vtj-connected path from $\mathcal{C}_{\sigma^{\prime}}(\sigma)$ to $\sigma^{\prime}$ such that does not intersect $\mathcal{X}^{s} \backslash\left\{\sigma, \sigma^{\prime}\right\}$. Formally,

$$
\begin{gathered}
\mathscr{U}_{\sigma^{\prime}}(\sigma):=\left\{\mathcal{C} \in \mathcal{M}\left(\mathcal{C}_{\left\{\sigma^{\prime}\right\}}^{+}(\sigma) \backslash\left\{\sigma^{\prime}\right\}\right) \mid \exists\left(\mathcal{C}_{1}, \ldots, \mathcal{C}_{m}\right) \in J_{\mathcal{C}_{\sigma^{\prime}}(\sigma),\left\{\sigma^{\prime}\right\}}\right. \text { such that } \\
\left.\bigcup_{i=1}^{m} \mathcal{C}_{i} \cap \mathcal{X}^{s} \backslash\left\{\sigma, \sigma^{\prime}\right\}=\varnothing \text { and } \exists j \in\{1, \ldots, n\}: \mathcal{C}_{j}=\mathcal{C}\right\} .
\end{gathered}
$$

Note that $\mathscr{U}_{\sigma^{\prime}}(\sigma)=\mathcal{M}\left(\mathcal{U}_{\sigma^{\prime}}(\sigma) \backslash\left(\mathcal{X}^{s} \backslash\{\sigma\}\right)\right)$ and that the boundary of $\mathcal{U}_{\sigma^{\prime}}(\sigma)$ consists of $\sigma^{\prime}$ and of states in the non-principal part of the boundary of some $\mathcal{C} \in \mathscr{U}_{\sigma^{\prime}}(\sigma)$ :

$$
\partial \mathcal{U}_{\sigma^{\prime}}(\sigma) \backslash\left\{\sigma^{\prime}\right\} \subseteq \bigcup_{\mathcal{C} \in \mathscr{U}_{\sigma^{\prime}}(\sigma)}(\partial \mathcal{C} \backslash \mathcal{B}(\mathcal{C}))=: \partial^{n p} \mathscr{U}_{\sigma^{\prime}}(\sigma) .
$$

For sake of semplicity, we will also refer to $\mathscr{U}_{\sigma^{\prime}}(\sigma)$ as restricted-tube of typical paths from $\sigma$ to $\sigma^{\prime}$.

Remark 4.1 Note that the notion of extended cyles is taken from [8]. In particular, using also the extended cycles for defining a cycle-path vtj-connected, we get that this object is the so-called standard cascade in [8].

\subsubsection{Model-Dependent Definitions and Notations}

- The union of all unit closed squares centered at the vertices $v \in V$ such that $\sigma(v)=s$ is denoted as $C^{s}(\sigma) \subseteq \mathbb{R}^{2}$. The $s$-clusters $C_{1}^{s}, C_{2}^{s}, \ldots$ are the connected components of 
$\mathrm{C}^{s}(\sigma)$, with the additional rule that we consider as distinct two $s$-clusters which share only one point. Two clusters $C_{i}^{s}, C_{j}^{s}$ are said to be interacting when either one of the following conditions is satisfied: (a) $C_{i}^{s}$ and $C_{j}^{s}$ intersect or (b) $C_{i}^{s}$ and $C_{j}^{s}$ are disjoint and there exists a site $v \notin C_{i}^{s} \cup C_{j}^{s}$ such that $\sigma(v) \neq s$ with two distinct nearest-neighbor sites $u, w$ lying inside $C_{i}^{s}, C_{j}^{s}$ respectively.

We say that a $q$-Potts configuration has $s$-interacting clusters when all its $s$-clusters are interacting.

- $R\left(C^{s}(\sigma)\right) \subseteq \mathcal{X}$ denotes the smallest rectangle that contains $\mathrm{C}^{s}(\sigma)$.

- For any $s, r \in\{1, \ldots, q\}$, let $\mathcal{X}(r, s):=\{\sigma \in \mathcal{X}: \sigma(v) \in\{r, s\} \forall v \in V\}$.

- Let $R_{\ell_{1} \times \ell_{2}}$ be a rectangle in $\mathbb{R}^{2}$ with horizontal side of length $\ell_{1}$ and vertical side of length $\ell_{2}$.

Now we define some extended cycles that are crucial to describe the tube of typical paths.

- Let

$\overline{\mathcal{K}}(r, s):=\{\sigma \in \mathcal{X}(r, s): H(\sigma)=2 K+2+H(\mathbf{r}), \sigma$ has either a $s$-cluster or

more $s$-interacting clusters and $\left.R\left(C^{s}(\sigma)\right)=R_{2 \times(K-1)}\right\} \cup \overline{\mathcal{Q}}(\mathbf{r}, \mathbf{s}) \cup \overline{\mathscr{P}}(\mathbf{r}, \mathbf{s})$.

Note that $\overline{\mathscr{H}}(\mathbf{r}, \mathbf{s}) \subset \overline{\mathcal{K}}(r, s)$.

- We set

$\overline{\mathcal{D}}_{1}(r, s):=\{\sigma \in \mathcal{X}(r, s): H(\sigma)=2 K+H(\mathbf{r}), \sigma$ has either a $s$-cluster

or more $s$-interacting clusters such that $\left.R\left(\mathrm{C}^{S}(\sigma)\right)=R_{2 \times(K-2)}\right\}$,

and

$\overline{\mathcal{E}}_{1}(r, s):=\{\sigma \in \mathcal{X}(r, s): H(\sigma)=2 K+H(\mathbf{r}), \sigma$ has either a $s$-cluster or

more $s$-interacting clusters such that $\left.R\left(\mathrm{C}^{s}(\sigma)\right)=R_{1 \times(K-1)}\right\} \cup \bar{R}_{1, K}(r, s)$.

- For $i=2, \ldots, K-2$, let

$\overline{\mathcal{D}}_{i}(r, s):=\{\sigma \in \mathcal{X}(r, s): H(\sigma)=2 K-2 i+2+H(\mathbf{r}), \sigma$ has either a $s$-cluster

or more $s$-interacting clusters such that $\left.R\left(\mathrm{C}^{S}(\sigma)\right)=R_{2 \times(K-(i+1))}\right\}$,

$\overline{\mathcal{E}}_{i}(r, s):=\{\sigma \in \mathcal{X}(r, s): H(\sigma)=2 K-2 i+2+H(\mathbf{r}), \sigma$ has either a $s$-cluster

or more $s$-interacting clusters such that $\left.R\left(\mathrm{C}^{S}(\sigma)\right)=R_{1 \times(K-i)}\right\}$.

- The sets $\widetilde{\mathcal{K}}(r, s), \widetilde{\mathcal{D}}_{i}(r, s), \widetilde{\mathcal{E}}_{i}(r, s)$ are defined analogously by interchanging the role of the spins $r$ and $s$.

We refer to Fig. 7 and to Figs. 13-15 for some examples of the extended cycles defined above.

\subsection{Main Results}

We are now ready to state the main results of this section. In order to understand Theorems 4.1, 4.2, 4.3, it is helpful to think of the following analogy. If Fig. 6 represented a top-down view of valleys having actual physical depth, and one imagines pouring a liquid in the valley corresponding to the configuration $\mathbf{1}$, then the tubes of typical trajectories are all the different ways in which the liquid might flow out of that valley. 


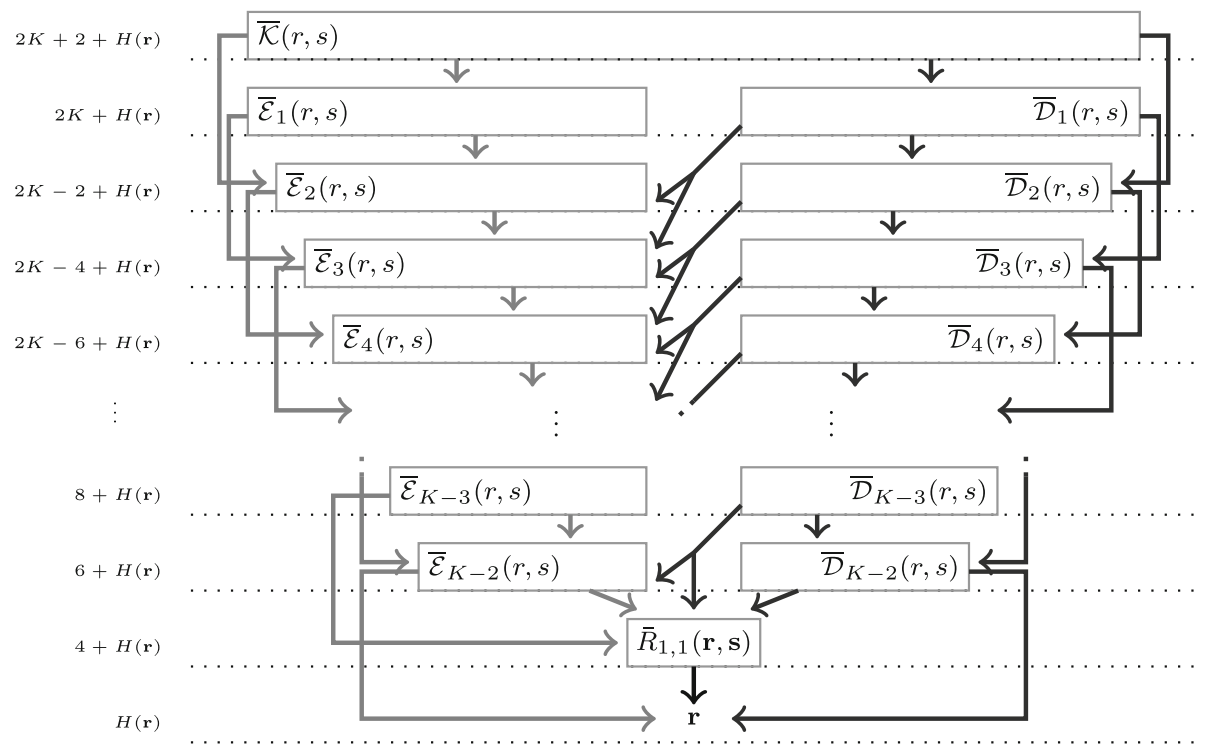

Fig. 7 Illustration of the first descent from $\overline{\mathcal{K}}(r, s)$ to $\mathbf{r}$. The rectangles denote extended cycles, i.e., the sets of trivial equielevated cycles. The arrows denote the connection between each extended cycle and the sets which belong to its principal boundary

\subsubsection{Restricted-Tube of Typical Paths Between Two Potts Stable Configurations}

Since in absence of external magnetic field the energy landscape between two Potts stable configurations is characterized by many extended-cycles, we describe the restricted-tube of typical paths defined in general in (4.12). For our model, let

$$
\begin{aligned}
\mathscr{U}_{\mathbf{s}}(\mathbf{r}):= & \bar{R}_{1,1}(r, s) \cup \bigcup_{i=1}^{K-2}\left(\overline{\mathcal{D}}_{i}(r, s) \cup \overline{\mathcal{E}}_{i}(r, s)\right) \cup \overline{\mathcal{K}}(r, s) \cup \bigcup_{h=2}^{K-2} \bar{B}_{1, K-1}^{h}(r, s) \\
& \cup \bigcup_{j=2}^{L-2 K-1} \bigcup_{h=1}^{L-2} \bar{B}_{j, K}^{h}(r, s) \cup \bigcup_{j=2}^{K-2} \bar{R}_{j, K}(r, s) \cup \bigcup_{h=2}^{K-2} \tilde{B}_{1, K-1}^{h}(r, s) \cup \widetilde{\mathcal{K}}(r, s) \\
& \cup \bigcup_{i=1}^{K-2}\left(\widetilde{\mathcal{D}}_{i}(r, s) \cup \widetilde{\mathcal{E}}_{i}(r, s)\right) \cup \tilde{R}_{1,1}(r, s) .
\end{aligned}
$$

As illustrated in the next result, $\mathscr{U}_{\mathbf{S}}(\mathbf{r})$ includes those configurations with nonzero probability of being visited by the Markov chain $\left\{X_{t}\right\}_{t \in \mathbb{N}}^{\beta}$ started in $\mathbf{r}$ before hitting $\mathbf{s}$ in the limit $\beta \rightarrow \infty$.

Theorem 4.1 (Restricted-tube of typical paths) For $\mathbf{r}, \mathbf{s} \in \mathcal{X}^{s}, \mathbf{s} \neq \mathbf{r}$, the $\operatorname{set} \mathscr{U}_{\mathbf{s}}(\mathbf{r})$ is the restricted-tube of typical paths for the transition $\mathbf{r} \rightarrow \mathbf{s}$. Moreover, there exists $k>0$ such that for $\beta$ sufficiently large

$$
\mathbb{P}_{\beta}\left(\tau_{\partial^{n p} \mathscr{U}_{\mathbf{s}}(\mathbf{r})}^{\mathbf{r}} \leq \tau_{\mathbf{s}}^{\mathbf{r}} \mid \tau_{\mathbf{s}}^{\mathbf{r}}<\tau_{\mathcal{X}^{s} \backslash\{\mathbf{r}, \mathbf{s}\}}^{\mathbf{r}}\right) \leq e^{-k \beta} .
$$




\subsubsection{Tube of Typical Paths Between a Stable State and the Other Stable States}

In this section we assume $q>2$, since for $q=2$ the Hamiltonian only has two global minima, and the tube of typical trajectories is given by Theorem 4.1. We recall the tube of typical paths defined in general in (4.9) and define $\mathfrak{T}_{\mathcal{X}^{s} \backslash\{\mathbf{r}\}}$ for Potts model with $q>2$ :

$$
\mathfrak{T}_{\mathcal{X}^{s} \backslash\{\mathbf{r}\}}(\mathbf{r}):=\bigcup_{\mathbf{t} \in \mathcal{X}^{s} \backslash\{\mathbf{r}\}} \mathscr{U}_{\mathbf{t}}(\mathbf{r})
$$

Theorem 4.2 (Tube of typical paths for the transition $\mathbf{r} \rightarrow \mathcal{X}^{s} \backslash\{\mathbf{r}\}$ ) For any $\mathbf{r} \in \mathcal{X}^{s}$, the set $\mathfrak{T}_{\mathcal{X}^{s} \backslash\{\mathbf{r}\}}(\mathbf{r})$ is the tube of typical trajectories for the transition $\mathbf{r} \rightarrow \mathcal{X}^{s} \backslash\{\mathbf{r}\}$ and there exists $k>0$ such that for $\beta$ sufficiently large

$$
\mathbb{P}_{\beta}\left(\tau_{\partial^{n p} \mathfrak{T}_{\mathcal{X}^{s} \backslash\{\mathbf{r}\}}^{\mathbf{r}}(\mathbf{r})} \leq \tau_{\mathcal{X}^{s} \backslash\{\mathbf{r}\}}^{\mathbf{r}}\right) \leq e^{-k \beta} .
$$

\subsubsection{Tube of Typical Paths Between a Stable State and Another Stable State}

We are now able to describe the tube of typical paths from a stable configuration to some other fixed stable state. We describe the typical trajectories for the transition $\mathbf{r} \rightarrow \mathbf{s}$ in terms of a sequence of transitions between two stable states such that the path followed by the process does not intersect other stable configurations. We recall the tube of typical paths defined in general in (4.9) and define $\mathfrak{T}_{\mathbf{s}}(\mathbf{r})$ for Potts model with $q>2$ :

$$
\mathfrak{T}_{\mathbf{s}}(\mathbf{r}):=\bigcup_{\mathbf{t} \in \mathcal{X}^{s} \backslash\{\mathbf{r}\}} \mathscr{U}_{\mathbf{t}}(\mathbf{r}) \cup \bigcup_{\mathbf{t}, \mathbf{t}^{\prime} \in \mathcal{X}^{s} \backslash\{\mathbf{r}, \mathbf{s}\}, \mathbf{t} \neq \mathbf{t}^{\prime}} \mathscr{U}_{\mathbf{t}}\left(\mathbf{t}^{\prime}\right) \cup \bigcup_{\mathbf{t}^{\prime} \in \mathcal{X}^{s} \backslash\{\mathbf{s}\}} \mathscr{U}_{\mathbf{s}}\left(\mathbf{t}^{\prime}\right) .
$$

Theorem 4.3 (Tube of typical paths for the transition $\mathbf{r} \rightarrow \mathbf{s}$ ) For any $\mathbf{r}, \mathbf{s} \in \mathcal{X}^{s}, \mathbf{r} \neq \mathbf{s}$ the set $\mathfrak{T}_{\mathbf{s}}(\mathbf{r})$ is the tube of typical trajectories for the transition $\mathbf{r} \rightarrow \mathbf{s}$ and there exists $k>0$ such that for $\beta$ sufficiently large

$$
\mathbb{P}_{\beta}\left(\tau_{\partial^{n p} \mathfrak{T}_{\mathbf{s}}(\mathbf{r})}^{\mathbf{r}} \leq \tau_{\mathbf{s}}^{\mathbf{r}}\right) \leq e^{-k \beta} .
$$

\subsubsection{Tube of Typical Paths for the Ising Model with Zero Magnetic Field}

For sake of concreteness, we give the following result on the tube of typical paths for the Ising model with zero magnetic field. We recall the tube of typical paths defined in general in (4.9) and define $\mathfrak{T}_{+\mathbf{1}}(\mathbf{- 1})$ for Ising model:

$$
\begin{aligned}
\mathfrak{T}_{+\mathbf{1}}(-\mathbf{1}):= & \bar{R}_{1,1}(-1,+1) \cup \bigcup_{i=1}^{K-2}\left(\overline{\mathcal{D}}_{i}(-1,+1) \cup \overline{\mathcal{E}}_{i}(-1,+1)\right) \cup \overline{\mathcal{K}}(-1,+1) \\
& \cup \bigcup_{h=2}^{K-2} \bar{B}_{1, K-1}^{h}(-1,+1) \cup \bigcup_{j=2}^{L-2 K-1} \bigcup_{h=1}^{h} \bar{B}_{j, K}^{h}(-1,+1) \cup \bigcup_{j=2}^{L-2} \bar{R}_{j, K}(-1,+1) \\
& \cup \bigcup_{h=2}^{K-2} \tilde{B}_{1, K-1}^{h}(-1,+1) \cup \widetilde{\mathcal{K}}(-1,+1) \cup \bigcup_{i=1}^{K-2}\left(\widetilde{\mathcal{D}}_{i}(-1,+1) \cup \widetilde{\mathcal{E}}_{i}(-1,+1) \cup \tilde{R}_{1,1}(-1,+1) .\right.
\end{aligned}
$$

Corollary 4.1 Consider the Ising model on a $K \times L$ grid $\Lambda$ with periodic boundary conditions and with zero external magnetic field. Then, we have that $\mathfrak{T}_{+\mathbf{1}}(-\mathbf{1})$ is the tube of typical 
trajectories for the transition $+\mathbf{1} \rightarrow-\mathbf{1}$ and there exists $k>0$ such that for $\beta$ sufficiently large

$$
\mathbb{P}_{\beta}\left(\tau_{\partial^{n p} \mathfrak{T}_{\{+\mathbf{1}\}}(-\mathbf{1})}^{\mathbf{r}} \leq \tau_{+\mathbf{1}}^{-\mathbf{1}}\right) \leq e^{-k \beta}
$$

\section{Minimal Restricted-Gates: Proofs}

In this section we prove our results on the set of minimal restricted-gates for the transition from $\mathbf{r} \in \mathcal{X}^{s}$ to $\mathbf{s} \in \mathcal{X}^{s}, \mathbf{r} \neq \mathbf{s}$. To this end, we first study the energy landscape between $\mathbf{r}$ and $\mathbf{s}$ by analyzing the optimal paths that do not visit other stable states.

\subsection{Energy Landscape Between Two Potts Stable Configurations}

In [1, Theorem 2.1], the authors prove that the communication energy (3.2) between any $\mathbf{r}, \mathbf{s} \in \mathcal{X}^{s}$, with $\mathbf{r} \neq \mathbf{s}$, is given by

$$
\Phi(\mathbf{r}, \mathbf{s})=2 \min \{K, L\}+2+H(\mathbf{r})=2 K+2+H(\mathbf{r}) .
$$

Hence, any optimal path $\omega \in \Omega_{\mathbf{r}, \mathbf{s}}^{o p t}$ does not contain configurations with energy strictly larger than $2 K+2+H(\mathbf{r})$.

Remark 5.1 For any $\sigma \in \mathcal{X}$ and every $\mathbf{r} \in \mathcal{X}^{s}$,

$$
H(\sigma)-H(\mathbf{r})=-\sum_{(v, w) \in E} \mathbb{1}_{\{\sigma(v)=\sigma(w)\}}+|E|=\sum_{(v, w) \in E} \mathbb{1}_{\{\sigma(v) \neq \sigma(w)\}} .
$$

Note that the total number of edges that connect two vertices with different spins, say $r \in S$ and $s \in S \backslash\{r\}$, in a configuration $\sigma \in \mathcal{X}$ is equal to the perimeter of the same-spin clusters in $C_{1}^{r}, C_{2}^{r}, \ldots$ Thus, thanks to (5.1) and (5.2), it follows that for any $\sigma$, that belongs to an optimal path $\omega \in \Omega_{\mathbf{r}, \mathbf{s}}^{\text {opt }}$, the total perimeter of its clusters with the same spin value cannot be larger than $2 K+2$.

The following lemma is an immediate consequence of (5.2).

Lemma 5.1 Consider $\mathbf{r}, \mathbf{s} \in \mathcal{X}^{s}, \mathbf{r} \neq \mathbf{s}$. Then, for every $j=1, \ldots, L-1$,

(a) The energy of any $\sigma \in \bar{B}_{j, K}^{h}(r, s)=\tilde{B}_{L-j-1, K}^{K-h}(r, s), h=1, \ldots, K-1, i s H(\sigma)=$ $H(\mathbf{s})+2 K+2=\Phi(\mathbf{r}, \mathbf{s})$.

(b) The energy of any $\sigma \in \bar{B}_{1, K-1}^{h}(r, s) \cup \tilde{B}_{1, K-1}^{h}(r, s), h=2, \ldots, K-2$, is such that $H(\sigma)=H(\mathbf{s})+2 K+2=\Phi(\mathbf{r}, \mathbf{s})$.

(c) The energy of any $\sigma \in \bar{R}_{j, K}(r, s)=\tilde{R}_{L-j, K}(r, s)$ is $H(\sigma)=H(\mathbf{s})+2 K$;

(d) The energy of any $\sigma \in \bar{R}_{j, K-1}(r, s) \cup \tilde{R}_{j, K-1}(r, s)$ is

$$
\begin{cases}H(\sigma)=H(\mathbf{s})+2 K, & \text { if } j=1 \\ H(\sigma)=H(\mathbf{s})+2 K+2=\Phi(\mathbf{r}, \mathbf{s}), & \text { if } j=2 \\ H(\sigma)>\Phi(\mathbf{r}, \mathbf{s}), & \text { if } j=3, \ldots, L-1 .\end{cases}
$$

The next lemma identifies which configurations communicate along an optimal path with the sets defined at the beginning of Sect. 3.1.2. For any $n=0, \ldots, K L$, let $N_{s}(\sigma):=\mid\{v \in V$ : $\sigma(v)=s\} \mid$ be the number of vertices with spin $s$ in the configuration $\sigma$. 
Lemma 5.2 Consider $\mathbf{r}, \mathbf{s} \in \mathcal{X}^{s}, \mathbf{r} \neq \mathbf{s}$. Given a configuration $\sigma$, let $\bar{\sigma}$ be a configuration which communicates with $\sigma$ along an optimal path from $\mathbf{r}$ to $\mathbf{s}$ that does not intersect $\mathcal{X}^{s} \backslash\{\mathbf{r}, \mathbf{s}\}$. For any $j=2, \ldots, L-2$, the following properties hold:

(a) if $\sigma \in \bar{B}_{j, K}^{h}(r, s)$ and $N_{s}(\sigma)>N_{s}(\bar{\sigma})$, then $\left\{\begin{array}{l}\bar{\sigma} \in \bar{R}_{j, K}(r, s) \text { if } h=1 ; \\ \bar{\sigma} \in \bar{B}_{j, K}^{h-1}(r, s), \text { if } h=2, \ldots, K-1 \text {; }\end{array}\right.$

(b) if $\sigma \in \bar{B}_{j, K}^{h}(r, s)$ and $N_{s}(\sigma)<N_{s}(\bar{\sigma})$, then $\left\{\begin{array}{l}\bar{\sigma} \in \bar{B}_{j, K}^{h+1}(r, s), \text { if } h=1, \ldots, K-2 \text {; } \\ \bar{\sigma} \in \bar{R}_{j+1, K}(r, s) \text {, if } h=K-1 \text {; }\end{array}\right.$

(c) if $\sigma \in \bar{R}_{j, K}(r, s)$ and $N_{s}(\sigma)>N_{s}(\bar{\sigma})$, then $\bar{\sigma} \in \bar{B}_{j-1, K}^{K-1}(r, s)$;

(d) if $\sigma \in \bar{R}_{j, K}(r, s)$ and $N_{s}(\sigma)<N_{s}(\bar{\sigma})$, then $\bar{\sigma} \in \bar{B}_{j, K}^{1}(r, s)$.

Proof Consider $\sigma \in \bar{B}_{j, K}^{h}(r, s)$ for some $j=2, \ldots, L-2, h=1, \ldots, K-1$. Let $\bar{\sigma}=\sigma^{v, t}$ for some $v \in V$ and some $t \in S$. Thanks to (2.6) the following implications hold:

(i) if $t \in S \backslash\{r, s\}$, then $H(\bar{\sigma})-H(\sigma) \geq 2$;

(ii) if $\sigma(v)=s, v$ has four nearest-neighbor spins $s, \bar{\sigma}(v)=r$, then $H(\bar{\sigma})-H(\sigma)=4$;

(iii) if $\sigma(v)=r, v$ has four nearest-neighbor spins $r, \bar{\sigma}(v)=s$, then $H(\bar{\sigma})-H(\sigma)=4$;

(iv) if $\sigma(v)=s, v$ has three nearest-neighbor spins $s, \bar{\sigma}(v)=r$, then $H(\bar{\sigma})-H(\sigma)=2$;

(v) if $\sigma(v)=r, v$ has three nearest-neighbor spins $r, \bar{\sigma}(v)=s$, then $H(\bar{\sigma})-H(\sigma)=2$.

Thanks to Lemma 5.1, in all the cases above we have $H(\bar{\sigma})>\Phi(\mathbf{r}, \mathbf{s})$, which contradicts the assumed optimality of the path. Hence, the only configurations $\bar{\sigma}$ that communicate with $\sigma \in \bar{B}_{j, K}^{h}(r, s)$ along an optimal path are those which are obtained by flipping either a spin from $s$ to $r$ or a spin from $r$ to $s$ among the spins with two nearest-neighbor spins $s$ and two nearest-neighbor spins $r$. In particular, following an optimal path from $\sigma \in \bar{B}_{j, K}^{h}(r, s)$ to $\mathbf{r}$ we have

$$
\begin{cases}\bar{\sigma} \in \bar{R}_{j, K}(r, s), & \text { if } h=1 \\ \bar{\sigma} \in \bar{B}_{j, K}^{h-1}(r, s), & \text { if } h=2, \ldots, K-1 .\end{cases}
$$

Similarly, following an optimal path from $\sigma \in \bar{B}_{j, K}^{h}(r, s)$ to $\mathbf{s}$, we have

$$
\begin{cases}\bar{\sigma} \in \bar{B}_{j, K}^{h+1}(r, s), & \text { if } h=1, \ldots, K-2 ; \\ \bar{\sigma} \in \bar{R}_{j+1, K}(r, s), & \text { if } h=K-1 .\end{cases}
$$

When $\sigma \in \bar{R}_{j, K}(r, s)$ for some $j=2, \ldots, L-2$, the proof proceeds similarly as above. Indeed, since by Lemma 5.1 we have that $H(\sigma)=2 K+H(\mathbf{r})$, the items (i)-(iii) above imply $H(\bar{\sigma})>\Phi(\mathbf{r}, \mathbf{s})$ which is not admissible. On the other hand, we obtain that the moves depicted in (iv) and (v) above are admissible and that starting from $\sigma \in \bar{R}_{j, K}(r, s)$, the next step in an optimal path to $\mathbf{r}$ is some $\bar{\sigma} \in \bar{B}_{j-1, K}^{K-1}(r, s)$, and the next step in an optimal path to $\mathbf{s}$ is some $\bar{\sigma} \in \bar{B}_{j, K}^{1}(r, s)$.

We remark that thanks to (3.5), Lemma 5.2 also describes the transition between those configurations which belong to either some $\tilde{B}_{j, K}^{h}(r, s)$ or some $\tilde{R}_{j, K}(r, s)$, for $j=2, \ldots, L-2$ and $h=1, \ldots, K-1$.

\subsection{Geometric Properties of the Potts Model with Zero External Magnetic Field}

A two dimensional polyomino on $\mathbb{Z}^{2}$ is a finite union of unit squares. The area of a polyomino is the number of its unit squares, while its perimeter is the cardinality of its boundary, namely, 


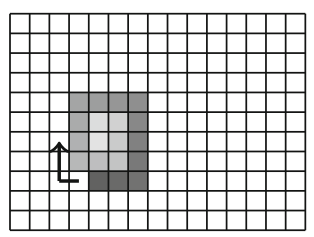

Fig. 8 First steps of path $\tilde{\omega}$ on a $11 \times 15$ grid $\Lambda$. The white squares have spin $r$, the other colors denote spin $s$. The arrow indicates the order in which the spins are flipped from $r$ to $s$. The colors of the squares indicate when they have been flipped, with darker squares having been flipped later

the number of unit edges of the dual lattice which intersect only one of the unit squares of the polyomino itself. Thus, the perimeter is the number of interfaces on $\mathbb{Z}^{2}$ between the sites inside the polyomino and those outside. We define $M_{n}$ as the set of all the polyominoes with minimal perimeter among those with area $n$. We call minimal polyominoes the elements of $M_{n}$. Next we prove some useful lemmas.

For any $n=0, \ldots, K L$, let $\mathcal{V}_{n}^{s}:=\left\{\sigma \in \mathcal{X}: N_{s}(\sigma)=n\right\}$ be the set of configurations with $n$ spins $s$. Note that every optimal path from any stable configuration $\mathbf{r} \neq \mathbf{s}$ to $\mathbf{s}$ necessarily intersects $\mathcal{V}_{n}^{s}$ for any $n=0, \ldots, K L$.

Lemma 5.3 Let $\mathbf{r}, \mathbf{s} \in \mathcal{X}^{s}$ be two different stable configurations and let $\omega$ be an optimal path for the transition from $\mathbf{r}$ to $\mathbf{s}$ such that $\omega \cap\left(\mathcal{X}^{s} \backslash\{\mathbf{r}, \mathbf{s}\}\right)=\varnothing$. There exists $K^{*} \in$ $\left\{0,1, \ldots,(K-1)^{2}\right\}$ such that in any $\sigma \in \omega$ with $K^{*}+1 \leq N_{s}(\sigma) \leq(K-1)^{2}$ at least a cluster of spins $s$ belongs to either $\bar{R}_{j, K}(r, s)$ or $\bar{B}_{j, K}^{h}(r, s)$, for some $\bar{h}=1, \ldots, K-1$ and $j=2, \ldots, L$. In other words, at least a cluster of spin $s$ wraps around $\Lambda$.

Proof The strategy for the proof is to construct a path $\tilde{\omega}: \mathbf{r} \rightarrow \tilde{\sigma}$ for some $\tilde{\sigma} \in$ $\bar{R}_{K-1, K-1}(r, s)$ as a sequence of configurations in which the unique cluster of spins $s$ is a polyomino (cluster that does not wrap around the torus) with minimal perimeter among those with the same area. After this construction we compare $\tilde{\omega}$ with any optimal path $\omega: \mathbf{r} \rightarrow \mathbf{s}$ up to $\tilde{\eta} \in \mathcal{V}_{(K-1)^{2}}^{s}$ with the aim to understand what is the last polyomino with perimeter smaller or equal than $2 K+2$, see Remark 5.1. In [48, Theorem 2.2] the authors show that the set of minimal polyominoes of area $n, M_{n}$, includes squares or quasi-squares with possibly a bar on one of the longest sides. Thus, we define $\tilde{\omega}:=\left(\tilde{\omega}_{0}, \tilde{\omega}_{1}, \tilde{\omega}_{2}, \ldots, \tilde{\sigma}\right)$ as the sequence of configurations having these shapes in which the number of spins $s$ increases following the clockwise direction, see Fig. 8 for an example of this construction.

Since $N_{s}\left(\tilde{\omega}_{j+1}\right)=N_{s}\left(\tilde{\omega}_{j}\right)+1$ for all $j=0,1, \ldots$, there exists some $K^{*}>0$ such that the perimeter of the $s$-cluster of $\tilde{\omega}_{K^{*}}$ is equal to $2 K+2$ and the perimeter of the $s$-cluster of $\tilde{\omega}_{K^{*}+1}$ is strictly larger than $2 K+2$. It follows that $H\left(\tilde{\omega}_{K^{*}}\right)=2 K+2+H(\mathbf{r})$ and $H\left(\tilde{\omega}_{K^{*}+1}\right)>2 K+2+H(\mathbf{r})$, thus $\tilde{\omega}_{K^{*}+1}$ does not belong to any $\omega \in \Omega_{\mathbf{r}, \mathbf{s}}^{\text {opt }}$. Explicit computations show that $K^{*}$ is given by

$$
K^{*}:= \begin{cases}\frac{K^{2}+2 K+1}{4}, & \text { if } K \text { is odd, } \\ \frac{K^{2}+2 K}{4}, & \text { if } K \text { is even. }\end{cases}
$$

If $K$ is odd (resp. even), the $s$-cluster of $\tilde{\omega}_{K^{*}}$ is a $\frac{K+1}{2} \times \frac{K+1}{2}$ square (resp. $\frac{K}{2} \times\left(\frac{K}{2}+1\right)$ rectangle). It follows that the configuration(s) in the intersection $\omega \cap \mathcal{V}_{n}^{s}$ for any $\omega \in \Omega_{\mathbf{r}, \mathbf{s}}^{o p t}$ and $K^{*}+1 \leq n \leq(K-1)^{2}$, do not contain any $\tilde{\omega}_{n}$ for any $n \geq K^{*}+1$. Hence, any optimal path intersects $\mathcal{V}_{n}^{s}$ with $n>K^{*}$ only in configurations with $s$-clusters wrapping around $\Lambda$. Moreover, the intersection belongs to either $\bar{R}_{j, K}(r, s)$ or $\bar{B}_{j, K}^{h}(r, s)$, for some $h=1, \ldots, K-1$ and $j=2, \ldots, L$. 
Lemma 5.4 Consider $\mathbf{r}, \mathbf{s} \in \mathcal{X}^{s}, \mathbf{r} \neq \mathbf{s}$. Then, for any $\omega \in \Omega_{\mathbf{r}, \mathbf{s}}^{\text {opt }}$ such that $\omega \cap\left(\mathcal{X}^{s} \backslash\{\mathbf{r}, \mathbf{s}\}\right)=$ $\varnothing$, we have

(a) $\omega \cap \overline{\mathscr{P}}(\mathbf{r}, \mathbf{s}) \neq \varnothing, \omega \cap \tilde{\mathscr{P}}(\mathbf{r}, \mathbf{s}) \neq \varnothing$;

(b) $\omega \cap \overline{\mathcal{Q}}(\mathbf{r}, \mathbf{s}) \neq \varnothing, \omega \cap \widetilde{\mathcal{Q}}(\mathbf{r}, \mathbf{s}) \neq \varnothing$;

(c) $\omega \cap \overline{\mathscr{H}}(\mathbf{r}, \mathbf{s}) \neq \varnothing, \omega \cap \mathscr{\mathscr { H }}(\mathbf{r}, \mathbf{s}) \neq \varnothing$.

Proof Using the reversibility of the dynamics, we prefer to study the optimal path $\omega \in \Omega_{\mathbf{r}, \mathbf{s}}^{o p t}$ such that $\omega \cap\left(\mathcal{X}^{s} \backslash\{\mathbf{r}, \mathbf{s}\}\right)=\varnothing$ by analyzing instead its time reversal $\omega^{T}=\left(\omega_{n}, \ldots, \omega_{0}\right)$. Indeed, a path $\omega=\left(\omega_{0}, \ldots, \omega_{n}\right)$ from $\mathbf{r}$ to $\mathbf{s}$ is optimal if and only if the path $\omega^{T}=$ $\left(\omega_{n}, \ldots, \omega_{0}\right)$ is optimal.

We begin by proving (a). We prove the statement only for $\overline{\mathscr{P}}(\mathbf{r}, \mathbf{s})$ since the proof for $\widetilde{\mathscr{P}}(\mathbf{r}, \mathbf{s})$ follows by switching the roles of $r$ and $s$ and using the symmetry of the model.

Let $\omega \in \Omega_{\mathbf{r}, \mathbf{s}}^{\text {opt }}$ be any optimal path between $\mathbf{r}$ and $\mathbf{s}$ such that $\omega \cap\left(\mathcal{X}^{s} \backslash\{\mathbf{r}, \mathbf{s}\}\right)=\varnothing$. Thanks to Lemma 5.3, there exists $K^{*} \in \mathbb{N}$ such that, when $n>K^{*}$, every $\omega$ intersects $\mathcal{V}_{n}^{s}$ in configurations which belong to either $\bar{B}_{j, K}^{h}(r, s)$ or $\bar{R}_{j, K}(r, s)$ for some $j=2, \ldots, L-2$ and $h=1, \ldots, K-1$. Moreover, by Lemma 5.2 it follows that $\omega$ reaches these configurations only moving among configurations belonging to either $\bar{B}_{j, K}^{h}(r, s)$ or $\bar{R}_{j, K}(r, s)$ with $j=$ $2, \ldots, L-2, h=1, \ldots, K-1$. In particular, the intersection $\omega \cap \bar{R}_{2, K}(r, s)$ is not empty. Take $\sigma \in \omega \cap \bar{R}_{2, K}(r, s)$. By Lemma 5.2(c), the only $\bar{\sigma} \in \mathcal{V}_{2 K-1}^{s}$ which communicates with $\sigma$ belongs to $\overline{\mathscr{P}}(\mathbf{r}, \mathbf{s})=\bar{B}_{1, K}^{K-1}(r, s)$.

Let us move to the proof of (b). To aid the understanding, we suggest to use Fig. 3 in Sect. 3.1.2 as a reference for this part of the proof. We prove the statement only for $\overline{\mathcal{Q}}(\mathbf{r}, \mathbf{s})$ since the proof for $\widetilde{\mathcal{Q}}(\mathbf{r}, \mathbf{s})$ again follows from symmetry considerations. Consider $\omega \in \Omega_{\mathbf{r}, \mathbf{s}}^{\text {opt }}$ such that $\omega \cap\left(\mathcal{X}^{s} \backslash\{\mathbf{r}, \mathbf{s}\}\right)=\varnothing$ and take $\sigma \in \overline{\mathscr{P}}(\mathbf{r}, \mathbf{s}) \cap \omega$ This exists in view of (a). Note that from Lemma 5.1 we have $H(\sigma)=\Phi(\mathbf{r}, \mathbf{s})$. Since $\sigma \in \mathcal{V}_{2 K-1}^{s}$, we have to move from $\sigma$ to $\bar{\sigma}$ by removing a spin $s$ and the only possibility to not overcome $\Phi(\mathbf{r}, \mathbf{s})$ is to change from $s$ to $r$ a spin $s$ with two nearest-neighbor spins $r$. Indeed, in a such a way the perimeter of the cluster with spins $s$ does not increase and $H(\bar{\sigma})$ does not exceed $\Phi(\mathbf{r}, \mathbf{s})$, see Remark 5.1. Hence, given $\sigma \in \overline{\mathscr{P}}(\mathbf{r}, \mathbf{s})$, the only configurations $\bar{\sigma} \in \mathcal{V}_{2 K-2}^{s}$ which communicate with $\sigma$, along an optimal path from $\sigma$ to $\mathbf{r}$, belong to either $\bar{R}_{2, K-1}(r, s)$ or $\bar{B}_{1, K}^{K-2}(r, s)$, which are subsets of $\overline{\mathcal{Q}}(\mathbf{r}, \mathbf{s})$.

Finally, we carry out the proof of (c). We prove the statement only for $\overline{\mathscr{H}}(\mathbf{r}, \mathbf{s})$ since the proof for $\widetilde{\mathscr{H}}(\mathbf{r}, \mathbf{s})$ again follows from symmetry considerations. For semplicity, we split the proof in several steps.

Step 1. We claim that, given $\bar{\sigma} \in \bar{R}_{2, K-1}(r, s) \cup \bar{B}_{1, K}^{K-2}(r, s)$, the only configurations $\hat{\sigma} \in$ $\mathcal{V}_{2 K-3}^{s}$ which communicate with $\bar{\sigma}$, along an optimal path from $\bar{\sigma}$ to $\mathbf{r}$, belong to either $\bar{B}_{1, K-1}^{K-2}(r, s)$ or $\bar{B}_{1, K}^{K-3}(r, s)$, see Fig. 9 .

We remark that $\bar{\sigma} \in \mathcal{V}_{2 K-2}^{s}$ and, thanks to Lemma 5.1, that $H(\bar{\sigma})=\Phi(\mathbf{r}, \mathbf{s})$. Hence, we have to move from $\bar{\sigma}$ to $\hat{\sigma}$ by removing a spin $s$ without increasing the energy and the only possibility is flipping from $s$ to $r$ a spin $s$ among those with two nearest-neighbor spins $s$. This can happen in several ways. Assume first that $\bar{\sigma} \in \bar{R}_{2, K-1}(r, s)$, then $\hat{\sigma} \in \bar{B}_{1, K-1}^{K-2}(r, s) \subset$ $\overline{\mathscr{H}}(\mathbf{r}, \mathbf{s})$ and both the proof of (c) and the proof of the claim are concluded. Otherwise, when $\bar{\sigma} \in \bar{B}_{1, K}^{K-2}(r, s)$, we flip from $s$ to $r$ either

(i) a spin $s$ with two nearest-neighbor spins $s$ which lies on the column full of $s$ or

(ii) a spin $s$ among those with two nearest-neighbor spins $s$ on the incomplete column of spins $s$. 
Fig. 9 Example on a $9 \times 12$ grid $\Lambda$ of Step 1. White vertices have spin $r$, gray vertices have spin $s$. Starting from $\bar{B}_{1, K}^{K-2}(r, s)$, the path can remain in $\overline{\mathcal{Q}}(\mathbf{r}, \mathbf{s})$ by flipping a spin $s$ to $r$ among those with dots, otherwise it can enter into $\overline{\mathscr{H}}(\mathbf{r}, \mathbf{s})$ by flipping from $s$ to $r$ a spin $s$ among those with lines. Note that from $\bar{B}_{1, K-1}^{K-3}(r, s)$ the path can enter

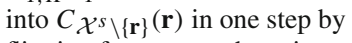
flipping from $s$ to $r$ the spin $s$ with three nearest-neighbor $r$

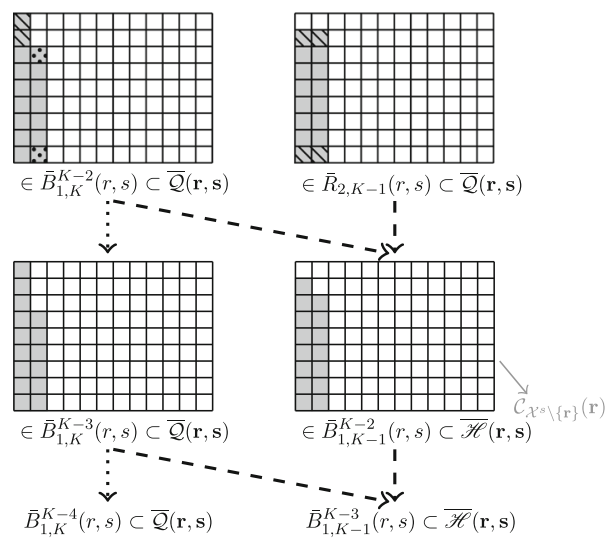

In particular, in case (i), $\hat{\sigma} \in \bar{B}_{1, K-1}^{K-2}(r, s) \subset \overline{\mathscr{H}}(\mathbf{r}, \mathbf{s})$ and both the proof of (c) and of the claim are completed. Otherwise, in case (ii), $\hat{\sigma} \in \bar{B}_{1, K}^{K-3}(r, s) \subset \overline{\mathcal{Q}}(\mathbf{r}, \mathbf{s})$. Thus claim is verified. However it is necessary to consider another step to prove (c).

Step 2. We claim that, given $\hat{\sigma} \in \bar{B}_{1, K}^{K-3}(r, s)$, the only configurations of $\mathcal{V}_{2 K-4}^{s}$ which communicate with $\hat{\sigma}$, along an optimal path between $\hat{\sigma}$ and $\mathbf{r}$, belong to either $\bar{B}_{1, K-1}^{K-3}(r, s)$ or $\bar{B}_{1, K}^{K-4}(r, s)$, see Fig. (4) in Sect. 3.1.2.

Since $H(\hat{\sigma})=\Phi(\mathbf{r}, \mathbf{s})$ (see Lemma 5.1), then in order to not increase the energy and to reduce the number of spins $s$, the moves (i) and (ii) of Step 1 are the only possibilities. It follows that $\omega$ can pass through $\bar{B}_{1, K}^{K-3}(r, s)$ coming from a configuration that lies in $\bar{B}_{1, K-1}^{K-3}(r, s) \subset \overline{\mathscr{H}}(\mathbf{r}, \mathbf{s})$ or in $\bar{B}_{1, K}^{K-4}(r, s) \subset \overline{\mathcal{Q}}(\mathbf{r}, \mathbf{s})$. In any case, the claim is verified. However, we conclude the proof of (c) only in the first case, otherwise we have to consider another step. Iterating the above construction, if at a certain step $\omega$ intersects $\mathcal{V}_{m}^{s}$, for $m=$ $K+2, \ldots, 2 K-2$, in a configuration of $\bar{B}_{1, K-1}^{n}(r, s) \subset \overline{\mathscr{H}}(\mathbf{r}, \mathbf{s})$ for some $n=3, \ldots, K-3$, then item (c) is satisfied and the proof is completed at that step. Otherwise, if $\omega$ intersects every $\mathcal{V}_{m}^{s}$, for $m=K+2, \ldots, 2 K-2$, in configurations belonging to $\overline{\mathcal{Q}}(\mathbf{r}, \mathbf{s})$, then the above construction leads to a configuration that lies in $\bar{B}_{1, K}^{2}(r, s) \subset \overline{\mathcal{Q}}(\mathbf{r}, \mathbf{s})$ and item (c) is satisfied because any $\eta \in \bar{B}_{1, K}^{2}(r, s)$ communicates with $\mathcal{V}_{K+1}^{s}$ only through configurations belonging to either $\bar{B}_{1, K}^{1}(r, s) \subset \overline{\mathscr{H}}(\mathbf{r}, \mathbf{s})$ or $\bar{B}_{1, K-1}^{2}(r, s) \subset \overline{\mathscr{H}}(\mathbf{r}, \mathbf{s})$, see Fig. 10. Indeed, (i) and (ii) of Step 1 are the only admissible options to move from $\bar{B}_{1, K}^{2}(r, s)$ to $\mathcal{V}_{K+1}^{s}$ following an optimal path.

In the proof of [1, Proposition 2.5], the authors define a reference path $\omega^{*}$ between any pair of different stable configurations of a $q$-state Potts model on a $K \times L$ grid $\Lambda$. Before stating the last lemma of the section, we briefly introduce this path. We say that a path $\omega: \sigma \rightarrow \sigma^{\prime}$ is the concatenation of the $L$ paths $\omega^{(i)}=\left(\omega_{0}^{(i)}, \ldots, \omega_{m_{i}}^{(i)}\right)$, for some $m_{i} \in \mathbb{N}, i=1, \ldots, L$, if $\omega=\left(\omega_{0}^{(1)}=\sigma, \ldots, \omega_{m_{1}}^{(1)}, \omega_{0}^{(2)}, \ldots, \omega_{m_{2}}^{(2)}, \ldots, \omega_{0}^{(L)}, \ldots, \omega_{m_{L}}^{(L)}=\sigma^{\prime}\right)$.

Definition 5.1 Given any $\mathbf{r}, \mathbf{s} \in \mathcal{X}^{s}, \mathbf{r} \neq \mathbf{s}$, the reference path $\omega^{*}$ is an optimal path from $\mathbf{r}$ to $\mathbf{s}$ that is formed by a sequence of configurations in which the cluster of spins $s$ grows gradually column by column. During the first $K$ steps, $\omega^{*}$ passes through configurations in which the spins on a particular column, say $c_{j}$ for some $j=0, \ldots, L-1$, become $s$, then it crosses those configurations in which the spins on either $c_{j+1}$ or $c_{j-1}$ become $s$ and so on. Without loss of generality the first spins that flip lie on the first column $c_{0}$. Formally, $\omega^{*}$ is 
Fig. 10 Example on a $9 \times 12$ grid $\Lambda$ of the final step of the proof of Lemma 5.4(c). White vertices have spin $r$, gray vertices have spin $s$. If the optimal path intersects $\mathcal{V}_{K+2}^{s}$ in a configuration of $\bar{B}_{1, K}^{2}(r, s) \subset \overline{\mathcal{Q}}(\mathbf{r}, \mathbf{s})$ and it has not already passed thorugh $\overline{\mathscr{H}}(\mathbf{r}, \mathbf{s})$, necessarily it arrives in this set by considering the step towards $\mathcal{V}_{K+1}^{s}$

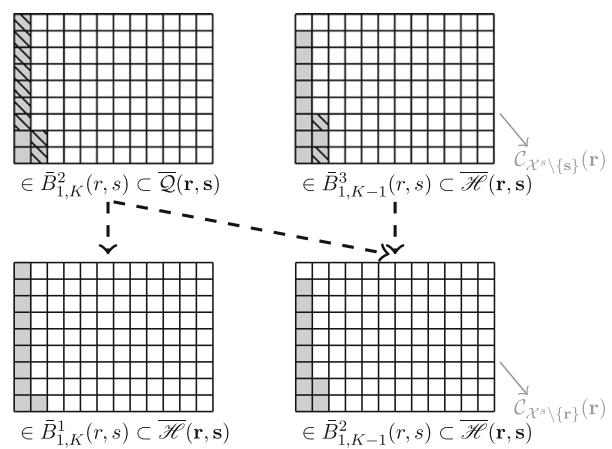

defined as the concatenation of $L$ paths $\omega^{*(1)}, \ldots, \omega^{*(L)}$ such that $\omega^{*(i)}: \eta_{i-1} \rightarrow \eta_{i}$, where $\eta_{0}:=\mathbf{r}, \eta_{L}:=\mathbf{s}$ and for any $i=1, \ldots, L, \eta_{i}$ is defined as

$$
\eta_{i}(v):= \begin{cases}s, & \text { if } v \in \bigcup_{j=0}^{i-1} c_{j}, \\ r, & \text { otherwise. }\end{cases}
$$

In particular, for any $i=1, \ldots, L$, we define $\omega^{*(i)}=\left(\omega_{0}^{*(i)}, \ldots, \omega_{K}^{*(i)}\right)$ as $\omega_{0}^{*(i)}=\eta_{i-1}$, $\omega_{h}^{*}{ }_{h}^{(i)}=\left(\omega_{h-1}^{*}\right)^{(h-1, i), s}$, for $h=1, \ldots, K-1$ and $\omega_{K}^{*(i)}=\eta_{i}$. Note that for any $i=$ $1, \ldots, L-1, h=1, \ldots, K-1$, we have $\omega_{h}^{*}{ }_{h}^{(1)} \in \bar{R}_{1, h}(r, s), \eta_{i} \in \bar{R}_{i, K}(r, s)$ and $\omega^{*}{ }_{h}^{(i+1)} \in$ $\bar{B}_{i, K}^{h}(r, s)$. Using Lemma 5.1 and the fact that $\Phi(\mathbf{r}, \mathbf{s})=2 K+2+H(\mathbf{r})$, we see, indeed, that $\omega^{*}$ is an optimal path.

Lemma 5.5 Let $\mathbf{r}, \mathbf{s} \in \mathcal{X}^{s}, \mathbf{r} \neq \mathbf{s}$. For any $\sigma \in \overline{\mathscr{H}}(\mathbf{r}, \mathbf{s})$ there exists a path $\bar{\omega}=\left(\bar{\omega}_{0}, \ldots, \bar{\omega}_{n}\right)$ from $\mathbf{r}$ to $\sigma$ such that

$$
H\left(\bar{\omega}_{i}\right)<2 K+2+H(\mathbf{r})
$$

for every $i=0, \ldots, n-1$. Similarly, there exists $\tilde{\omega}$ from $\mathbf{s}$ to any $\sigma \in \widetilde{\mathscr{H}}(\mathbf{r}, \mathbf{s})$ with the same properties of $\bar{\omega}$.

Proof We prove that there exists $\bar{\omega}: \mathbf{r} \rightarrow \sigma$ which satisfies (5.8) for any $\sigma \in \overline{\mathscr{H}}(\mathbf{r}, \mathbf{s})$; by reversing the roles of $r$ and $s$, the proof of the existence of $\tilde{\omega}$ from $\mathbf{s}$ to any $\sigma \in \widetilde{\mathscr{H}}(\mathbf{r}, \mathbf{s})$ is analogous. The definition of $\overline{\mathscr{H}}(\mathbf{r}, \mathbf{s})$ gives rise to the two following scenarios, see (3.8). If $\sigma \in \bar{B}_{1, K}^{1}(r, s)$, then $\bar{\omega}$ is given by the first steps of the path $\omega^{*}$ depicted in Definition 5.1. Indeed, without loss of generality, we rename the column in which $\sigma$ has all spins $s$ as $c_{0}$ and we set $\bar{\omega}=\left(\mathbf{r}, \omega_{1}^{*}{ }_{1}^{(1)}, \ldots, \omega_{K}^{*(1)}, \omega_{1}^{*(2)}=\sigma\right)$. If $\sigma \in \bar{B}_{1, K-1}^{h}(r, s)$ for some $h=2, \ldots, K-2$, we proceed as follows. Possibly relabeling the columns, we build $\bar{\omega}$ taking into account the columns $c_{0}, c_{1}$ of the grid. In Fig. 11 we depict an example of the path $\bar{\omega}$. For every $h=2, \ldots, K-2$ and for any odd value $i$ from 1 to $2 h-1$, we set

$$
\bar{\omega}_{i}=\bar{\omega}_{i-1}^{\left(\frac{i-1}{2}, 0\right), s}, \bar{\omega}_{i+1}=\bar{\omega}_{i}^{\left(\frac{i-1}{2}, 1\right), s} \text {. }
$$

Then, we set

$$
\bar{\omega}_{j}=\bar{\omega}_{j-1}^{(j-h, 0)},
$$

for any $j=2 h+1, \ldots, K-1+h$. As we see in Fig. 11, after $2 h$ steps $\bar{\omega}$ arrives in $\bar{\omega}_{2 h} \in$ $\bar{R}_{2, h}(r, s)$ and its next configurations belong to $\bar{B}_{1, j-h}^{h}(r, s)$ for $j=2 h+1, \ldots, K-1+h$. Finally, (5.8) is satisfied in view of (5.2). 

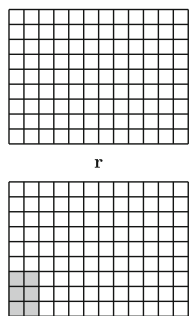

$\bar{\omega}_{6}$

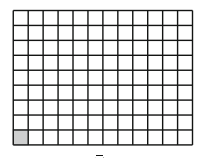

$\bar{\omega}_{1}$

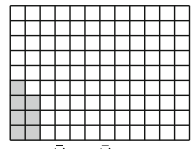

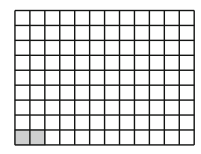

$\bar{\omega}_{2}$

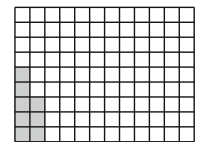

$\bar{\omega}_{8}$

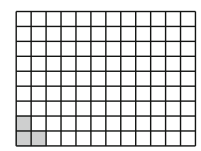

$\bar{\omega}_{3}$

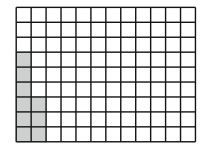

$\bar{\omega}_{9}$

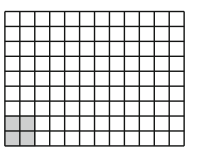

$\bar{\omega}_{4}$

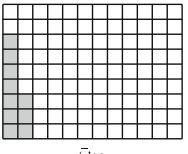

$\bar{\omega}_{10}$

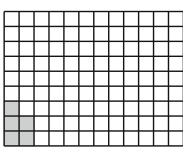

$\bar{\omega}_{5}$

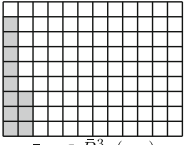

$\bar{\omega}_{11} \in \bar{B}_{1,8}^{3}(r, s)$

Fig. 11 Example of $\bar{\omega}: \mathbf{r} \rightarrow \sigma$ of Lemma 5.5 where $\sigma \in \bar{B}_{1, K-1}^{h}(r, s)$ with $K=9$ and $h=3$. White vertices have spin $r$, gray vertices have spin $s$

\subsection{Study of the Set of All Minimal Restricted-Gates Between Two Different Stable States}

We are now able to prove the following results concerning the set of minimal restricted-gates from $\mathbf{r} \in \mathcal{X}^{s}$ to $\mathbf{s} \in \mathcal{X}^{s}, \mathbf{s} \neq \mathbf{r}$.

Proof of Theorem 3.1. In order to prove that a set $\mathcal{W}_{\mathrm{RES}} \subset \mathcal{S}(\mathbf{r}, \mathbf{s})$ is a minimal restricted-gate for the transition from $\mathbf{r}$ to $\mathbf{s}$ we show that

(i) $\mathcal{W}_{\text {RES }}$ is a restricted-gate, i.e., every $\omega \in \Omega_{\mathbf{r}, \mathbf{s}}^{\text {opt }}$ such that $\omega \cap\left(\mathcal{X}^{s} \backslash\{\mathbf{r}, \mathbf{s}\}\right)=\varnothing$ intersects $\mathcal{W}_{\text {RES }}$

(ii) for any $\eta \in \mathcal{W}_{\text {RES }}$ there exists an optimal path $\omega^{\prime} \in \Omega_{\mathbf{r}, \mathbf{s}}^{\text {opt }}$ such that $\omega^{\prime} \cap\left(\mathcal{W}_{\mathrm{RES}} \backslash\{\eta\}\right)=\varnothing$.

Hence, we now show that the sets defined in (a), (b), (c) and (d) of Theorem 3.1 satisfy the conditions above. Using Lemma 5.4(a), $\overline{\mathscr{P}}(\mathbf{r}, \mathbf{s})$ and $\mathscr{\mathscr { P }}(\mathbf{r}, \mathbf{s})$ are gates for the transition from $\mathbf{r}$ to $\mathbf{s}$. Next let us show that for any $\eta \in \overline{\mathscr{P}}(\mathbf{r}, \mathbf{s})$ there exists an optimal path $\omega^{\prime} \in \Omega_{\mathbf{r}, \mathbf{s}}^{\text {opt }}$ such that $\omega^{\prime} \cap(\overline{\mathscr{P}}(\mathbf{r}, \mathbf{s}) \backslash\{\eta\})=\varnothing$. It is enough to consider $\omega^{\prime}$ as the path $\omega^{*}$ of Definition 5.1 and to rewrite it in order to have $\omega^{\prime} \cap \overline{\mathscr{P}}(\mathbf{r}, \mathbf{s})=\{\eta\}$, i.e., $\omega_{K-1}^{*(2)}=\eta$. By the symmetry of the model, we prove similarly that there exists a such a path also for any $\eta \in \widetilde{\mathscr{P}}(\mathbf{r}, \mathbf{s})$.

Using Lemma 5.4(b), $\overline{\mathcal{Q}}(\mathbf{r}, \mathbf{s})$ and $\widetilde{\mathcal{Q}}(\mathbf{r}, \mathbf{s})$ are gates for the transition from $\mathbf{r}$ to $\mathbf{s}$. Next let us show that for any $\eta \in \overline{\mathcal{Q}}(\mathbf{r}, \mathbf{s})$ there exists an optimal path $\omega^{\prime} \in \Omega_{\mathbf{r}, \mathbf{s}}^{\text {opt }}$ such that $\omega^{\prime} \cap(\overline{\mathcal{Q}}(\mathbf{r}, \mathbf{s}) \backslash\{\eta\})=\varnothing$. We distinguish two cases:

(i) if $\eta \in \bar{R}_{2, K-1}(r, s)$, given $\bar{\eta} \in \bar{B}_{1, K-1}^{K-2}(r, s)$ and $\hat{\eta} \in \overline{\mathscr{P}}(\mathbf{r}, \mathbf{s})$ which communicate with $\eta$, then $\omega^{\prime}$ is the path given by the concatenation of

- the path $\bar{\omega}: \mathbf{r} \rightarrow \bar{\eta}$ of Lemma 5.5;

- the path $(\bar{\eta}, \eta, \hat{\eta})$;

- the portion of the path $\omega^{*}$ in Definition 5.1 from $\omega_{K-1}^{*(2)}=\hat{\eta}$ to $\mathbf{s}$,

so that $\omega^{\prime} \cap \overline{\mathcal{Q}}(\mathbf{r}, \mathbf{s})=\{\eta\}$;

(ii) if $\eta \in \bar{B}_{1, K}^{h}(r, s)$, for some $h=2, \ldots, K-2$, then to define $\omega^{\prime}$ it is enough to consider the path $\omega^{*}$ of Definition 5.1 and to construct it in order to have $\omega^{\prime} \cap \overline{\mathcal{Q}}(\mathbf{r}, \mathbf{s})=\{\eta\}$, i.e., $\omega_{h}^{*}=\eta$.

Thanks to the symmetry of the model, we define $\omega^{\prime}$ in an analogous way for any $\eta \in \widetilde{\mathcal{Q}}(\mathbf{r}, \mathbf{s})$.

Using Lemma 5.4(c), $\overline{\mathscr{H}}(\mathbf{r}, \mathbf{s})$ and $\widetilde{\mathscr{H}}(\mathbf{r}, \mathbf{s})$ are gates for the transition from $\mathbf{r}$ to $\mathbf{s}$. Next let us show that for any $\eta \in \overline{\mathscr{H}}(\mathbf{r}, \mathbf{s})$ there exists an optimal path $\omega^{\prime} \in \Omega_{\mathbf{r}, \mathbf{s}}^{o p}$ such that $\omega^{\prime} \cap(\overline{\mathscr{H}}(\mathbf{r}, \mathbf{s}) \backslash\{\eta\})=\varnothing$. We distinguish two cases: 
(i) if $\eta \in \bar{B}_{1, K}^{1}(r, s)$, then $\omega^{\prime}$ is given by the path $\omega^{*}$ of Definition 5.1 defined in order to have $\omega^{\prime} \cap \overline{\mathscr{H}}(\mathbf{r}, \mathbf{s})=\{\eta\}$, i.e., $\omega_{1}^{*}{ }_{1}^{(2)}=\eta$;

(ii) if $\eta \in \bar{B}_{1, K-1}^{h}(r, s)$, for some $h=2, \ldots, K-2$, then $\omega^{\prime}$ corresponds to the optimal path given by the concatenation of

- the path $\bar{\omega}: \mathbf{r} \rightarrow \eta$ of Lemma 5.5;

- the path $(\eta, \bar{\eta})$ with $\bar{\eta} \in \bar{B}_{1, K}^{h}(r, s)$, such that the bar of length $h$ is in the same position as in $\eta$;

- the portion of the path $\omega^{*}$ in Definition 5.1 from $\omega_{h}^{*}(2)=\bar{\eta}$ to $\mathbf{s}$,

so that $\omega^{\prime} \cap \overline{\mathscr{H}}(\mathbf{r}, \mathbf{s})=\{\eta\}$.

Thanks to the symmetry of the model, we define $\omega^{\prime}$ for any $\eta \in \widetilde{\mathscr{H}}(\mathbf{r}, \mathbf{s})$ following the same strategy.

From Lemma 5.3 and Lemma 5.2, we conclude that $\mathcal{W}_{j}^{(h)}(\mathbf{r}, \mathbf{s})$ are gates for the transition $\mathbf{r} \rightarrow \mathbf{s}$ for any $j=2, \ldots, L-3$ and any $h=1, \ldots, K-1$. Indeed, by Lemma 5.3, there exists $K^{*} \in \mathbb{N}$ such that when $n>K^{*}$ every $\omega \in \Omega_{\mathbf{r}, \mathbf{s}}^{\text {opt }}$ intersects $\mathcal{V}_{n}^{s}$ in configurations which belong to either $\bar{R}_{j, K}(r, s)$ or $\mathcal{W}_{j}^{(h)}(\mathbf{r}, \mathbf{s})=\bar{B}_{j, K}^{h}(r, s)$ for some $j=2, \ldots, L-3, h=$ $1, \ldots, K-1$. Moreover, by Lemma $5.2, \omega$ reaches these configurations only moving among configurations lying either in $\bar{R}_{j, K}(r, s)$ or in $\mathcal{W}_{j}^{(h)}(\mathbf{r}, \mathbf{s})=\bar{B}_{j, K}^{h}(r, s)$. Hence, between its last visit to $\overline{\mathscr{P}}(\mathbf{r}, \mathbf{s})$ and its first visit to $\widetilde{\mathscr{P}}(\mathbf{r}, \mathbf{s}), \omega$ passes at least once through each $\mathcal{W}_{j}^{(h)}(\mathbf{r}, \mathbf{s})$, $j=2, \ldots, L-3$. Thus, to conclude the proof we show that for every $\eta \in W_{j}^{(h)}(\mathbf{r}, \mathbf{s})$, there exists a path $\omega^{\prime} \in \Omega_{\mathbf{r}, \mathbf{s}}^{o p t}$ such that $\omega^{\prime} \cap\left(\mathcal{W}_{j}^{(h)}(\mathbf{r}, \mathbf{s}) \backslash\{\eta\}\right)=\varnothing$. For any $j=2, \ldots, L-3$ and any $h=1, \ldots, K-1$, we define $\omega^{\prime}$ as the path $\omega^{*}$ of Definition 5.1, which we rewrite in order to have $\omega^{\prime} \cap \mathcal{W}_{j}^{(h)}(\mathbf{r}, \mathbf{s})=\{\eta\}$, i.e., $\omega_{h}^{*}{ }_{h}^{(j+1)}=\eta$.

Remark 5.2 A saddle $\eta \in \mathcal{S}\left(\sigma, \sigma^{\prime}\right)$ is unessential if for any $\omega \in \Omega_{\sigma, \sigma^{\prime}}^{o p t}$ such that $\omega \cap \eta \neq \varnothing$ the following conditions are both satisfied:

(a) $\left\{\operatorname{argmax}_{\omega} H\right\} \backslash\{\eta\} \neq \varnothing$,

(b) there exists $\omega^{\prime} \in \Omega_{\sigma, \sigma^{\prime}}^{\text {opt }}$ such that $\left\{\operatorname{argmax}_{\omega^{\prime}} H\right\} \subseteq\left\{\operatorname{argmax}_{\omega} H\right\} \backslash\{\eta\}$.

Proof of Theorem 3.2. In view of Theorem 3.1, we have

$$
\bigcup_{j=2}^{L-3} \mathcal{W}_{j}(\mathbf{r}, \mathbf{s}) \cup \overline{\mathscr{H}}(\mathbf{r}, \mathbf{s}) \cup \widetilde{\mathscr{H}}(\mathbf{r}, \mathbf{s}) \cup \overline{\mathcal{Q}}(\mathbf{r}, \mathbf{s}) \cup \widetilde{\mathcal{Q}}(\mathbf{r}, \mathbf{s}) \cup \overline{\mathscr{P}}(\mathbf{r}, \mathbf{s}) \cup \widetilde{\mathscr{P}}(\mathbf{r}, \mathbf{s}) \subseteq \mathcal{F}(\mathbf{r}, \mathbf{s}) .
$$

Hence, we are left to prove the opposite inclusion. In order to do this, we use the characterization of minimal gates as essential saddles given in [3, Theorem 5.1]. More precisely, we prove that any

$$
\eta \in \mathcal{S}(\mathbf{r}, \mathbf{s}) \backslash\left[\bigcup_{j=2}^{L-3} \mathcal{W}_{j}(\mathbf{r}, \mathbf{s}) \cup \overline{\mathscr{H}}(\mathbf{r}, \mathbf{s}) \cup \widetilde{\mathscr{H}}(\mathbf{r}, \mathbf{s}) \cup \overline{\mathcal{Q}}(\mathbf{r}, \mathbf{s}) \cup \widetilde{\mathcal{Q}}(\mathbf{r}, \mathbf{s}) \cup \overline{\mathscr{P}}(\mathbf{r}, \mathbf{s}) \cup \widetilde{\mathscr{P}}(\mathbf{r}, \mathbf{s})\right]
$$

is an unessential saddle for the restricted transition from $\mathbf{r}$ to $\mathbf{s}$. Consider $\eta$ as in (5.9) and some $\omega \in \Omega_{\mathbf{r}, \mathbf{s}}^{o p t}, \omega=\left(\omega_{0}, \ldots, \omega_{n}\right)$, such that $\omega \cap \mathcal{X}^{s} \backslash\{\mathbf{r}, \mathbf{s}\}=\varnothing$ and $\eta \in \omega$. See Fig. 12 for an example of $\omega$. By Lemmas 5.2 and 5.1 the condition (i) of Remark 5.2 is satisfied. Indeed, any $\omega \in \Omega_{\mathbf{r}, \mathbf{s}}^{\text {opt }}$ passes through many configurations with energy value equal to $\Phi(\mathbf{r}, \mathbf{s})$. Hence, to conclude that $\eta$ is an unessential saddle we are left to verify condition (b) of Remark 5.2. 


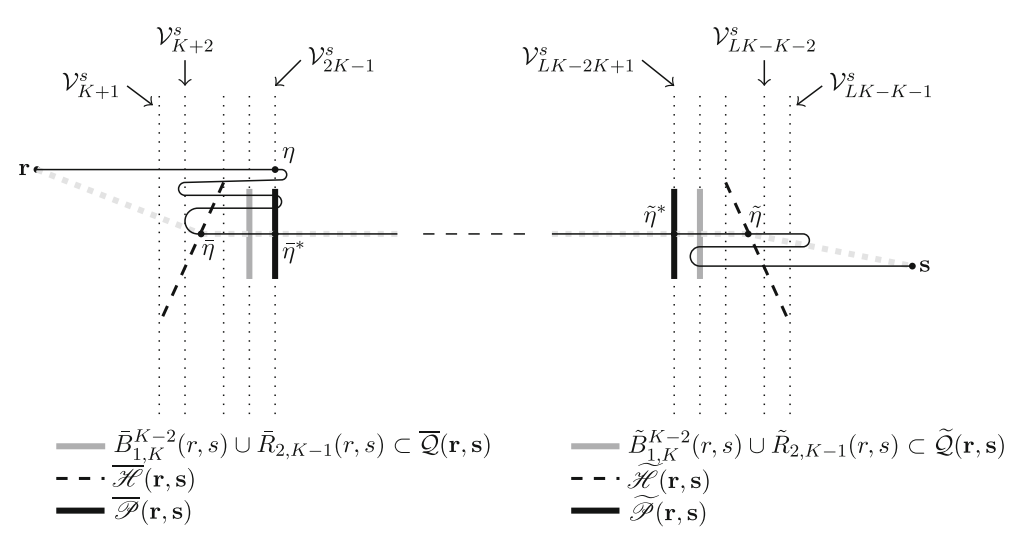

Fig. 12 Example of the paths $\omega$ (solid black path) and $\omega^{\prime}$ (dotted gray path) of the proof of Theorem 3.6

By Lemma 5.4(c), there exist $\bar{\eta} \in \omega \cap \overline{\mathscr{H}}(\mathbf{r}, \mathbf{s})$ and $\tilde{\eta} \in \omega \cap \widetilde{\mathscr{H}}(\mathbf{r}, \mathbf{s})$, where $\bar{\eta}$ is the last configuration visited by $\omega$ in $\overline{\mathscr{H}}(\mathbf{r}, \mathbf{s})$ and $\tilde{\eta}$ is the first configuration visited by $\omega$ in $\widetilde{\mathscr{H}}(\mathbf{r}, \mathbf{s})$. Moreover, Lemma 5.4(a) implies that there exist $\bar{\eta}^{*} \in \omega \cap \overline{\mathscr{P}}(\mathbf{r}, \mathbf{s})$ and $\tilde{\eta}^{*} \in \omega \cap \widetilde{\mathscr{P}}(\mathbf{r}, \mathbf{s})$, where $\bar{\eta}^{*}$ is the last configuration visited by $\omega$ in $\overline{\mathscr{P}}(\mathbf{r}, \mathbf{s})$ and $\tilde{\eta}^{*}$ is the first configuration visited by $\omega$ in $\widetilde{\mathscr{P}}(\mathbf{r}, \mathbf{s})$.

In view of the proof of Lemma 5.4, after visiting $\bar{\eta}, \omega$ interstects $\mathcal{S}(\mathbf{r}, \mathbf{s})$ only in saddles belonging to either $\overline{\mathcal{Q}}(\mathbf{r}, \mathbf{s})$ or $\overline{\mathscr{P}}(\mathbf{r}, \mathbf{s})$ or $\mathcal{W}_{j}(\mathbf{r}, \mathbf{s})$, for some $j=2, \ldots, L-3$, until it intersects $\widetilde{\mathscr{P}}(\mathbf{r}, \mathbf{s})$ in $\tilde{\eta}^{*}$. Similarly, after the visit in $\bar{\eta}^{*}$ and before the arrival in $\tilde{\eta}, \omega$ passes only through saddles belonging to either $\mathcal{W}_{j}(\mathbf{r}, \mathbf{s})$, for some $j=2, \ldots, L-3$, or $\widetilde{\mathscr{P}}(\mathbf{r}, \mathbf{s})$ or $\widetilde{\mathcal{Q}}(\mathbf{r}, \mathbf{s})$. It follows that after $\bar{\eta}$ and before $\tilde{\eta}, \omega$ intersects $\mathcal{S}(\mathbf{r}, \mathbf{s})$ only in those saddles which belong to $\bigcup_{j=2}^{L-3} \mathcal{W}_{j}(\mathbf{r}, \mathbf{s}) \cup \overline{\mathscr{H}}(\mathbf{r}, \mathbf{s}) \cup \widetilde{\mathscr{H}}(\mathbf{r}, \mathbf{s}) \cup \overline{\mathcal{Q}}(\mathbf{r}, \mathbf{s}) \cup \widetilde{\mathcal{Q}}(\mathbf{r}, \mathbf{s}) \cup \overline{\mathscr{P}}(\mathbf{r}, \mathbf{s}) \cup \widetilde{\mathscr{P}}(\mathbf{r}, \mathbf{s})$.

Now consider the paths $\bar{\omega}: \mathbf{r} \rightarrow \bar{\eta}$ and $\tilde{\omega}: \mathbf{s} \rightarrow \tilde{\eta}$, which exist in view of Lemma 5.5, and take the time reversal of $\tilde{\omega}$, i.e., $\tilde{\omega}^{T}=\left(\omega_{n}=\tilde{\eta}, \tilde{\omega}_{n-1}, \ldots, \omega_{1}, \omega_{0}=\mathbf{s}\right)$. Thus, if $\omega=\left(\omega_{0}=\mathbf{r}, \ldots, \omega_{i}=\bar{\eta}, \ldots, \omega_{j}=\tilde{\eta}, \ldots, \omega_{n}=\mathbf{s}\right)$, the path $\omega^{\prime} \in \Omega_{\mathbf{r}, \mathbf{s}}^{\text {opt }}$, may be defined as $\omega^{\prime} \equiv \bar{\omega}$ from $\mathbf{r}$ to $\bar{\eta}, \omega^{\prime} \equiv\left(\omega_{i}=\bar{\eta}, \ldots, \omega_{j}=\tilde{\eta}\right)$ from $\bar{\eta}$ to $\tilde{\eta}$, and $\omega^{\prime} \equiv \tilde{\omega}^{T}$ from $\tilde{\eta}$ to $\mathbf{s}$. Thus, condition (b) of Remark 5.2 is verified.

Proof of Corollary 3.1. The corollary follows from Theorem 3.1 and [3, Theorem 5.4].

\section{Minimal Gates: Proofs}

We are now able to carry out the proof of the main results on the minimal gates for the transitions from a stable state to the other stable configurations and from a stable state to another stable configuration.

\subsection{The Minimal Gates from a Stable State to the Other Stable States}

Proof of Theorem 3.3. We begin by proving that the sets in (a) are minimal gates. Consider any $\omega \in \Omega_{\mathbf{r}, \mathcal{X}^{s} \backslash\{\mathbf{r}\}}^{o p t}$ and let $\mathbf{s} \in \mathcal{X}^{s} \backslash\{\mathbf{r}\}$ be the first configuration visited by $\omega$ in $\mathcal{X}^{s} \backslash\{\mathbf{r}\}$. From 
Theorem 3.1(a) we have that $\omega \cap \overline{\mathscr{P}}(\mathbf{r}, \mathbf{s}) \neq \varnothing$ and $\omega \cap \widetilde{\mathscr{P}}(\mathbf{r}, \mathbf{s}) \neq \varnothing$. Thus,

$$
\omega \cap\left(\bigcup_{\mathbf{t} \in \mathcal{X}^{s} \backslash\{\mathbf{r}\}} \overline{\mathscr{P}}(\mathbf{r}, \mathbf{t})\right) \neq \varnothing \text { and } \omega \cap\left(\bigcup_{\mathbf{t} \in \mathcal{X}^{s} \backslash\{\mathbf{r}\}} \tilde{\mathscr{P}}(\mathbf{r}, \mathbf{t})\right) \neq \varnothing
$$

hence these sets are gates. Moreover, for any $\eta \in \bigcup_{\mathbf{t} \in \mathcal{X}^{s} \backslash\{\mathbf{r}\}} \overline{\mathscr{P}}(\mathbf{r}, \mathbf{t})$ there exists $\mathbf{s} \in \mathcal{X}^{s} \backslash\{\mathbf{r}\}$ such that $\eta \in \overline{\mathscr{P}}(\mathbf{r}, \mathbf{s})$. The optimal path $\omega^{\prime} \in \Omega_{\mathbf{r}, \mathcal{X}^{s} \backslash\{\mathbf{r}\}}^{o p t}$ from $\mathbf{r}$ to $\mathbf{s} \in \mathcal{X}^{s} \backslash\{\mathbf{r}\}$ that was constructed in the proof of Theorem 3.1(a) was such that $\omega^{\prime} \cap\left(\bigcup_{\mathbf{t} \in \mathcal{X}^{s} \backslash\{\mathbf{r}\}} \overline{\mathscr{P}}(\mathbf{r}, \mathbf{t}) \backslash\{\eta\}\right)=\varnothing$ and $\omega^{\prime} \cap \mathcal{X}^{s} \backslash\{\mathbf{r}, \mathbf{s}\}=\varnothing$. Hence, $\bigcup_{\mathbf{t} \in \mathcal{X}^{s} \backslash\{\mathbf{r}\}} \overline{\mathscr{P}}(\mathbf{r}, \mathbf{t})$ is a minimal gate. By the symmetry of the model, the same holds for $\bigcup_{\mathbf{t} \in \mathcal{X}^{s} \backslash\{\mathbf{r}\}} \widetilde{\mathscr{P}}(\mathbf{r}, \mathbf{t})$.

The proof for the sets (b), (c) and (d) proceeds analogously by exploiting the path construction in the proof of Theorem 3.1(b), (c), (d), respectively.

Proof of Theorem 3.4. In view of Theorem 3.3 we have

$$
\begin{aligned}
\bigcup_{\mathbf{t} \in \mathcal{X}^{s} \backslash\{\mathbf{r}\}}\left[\bigcup_{j=2}^{L-3} \mathcal{W}_{j}(\mathbf{r}, \mathbf{t}) \cup \overline{\mathscr{H}}(\mathbf{r}, \mathbf{t}) \cup \widetilde{\mathscr{P}}(\mathbf{r}, \mathbf{t}) \cup \overline{\mathcal{Q}}(\mathbf{r}, \mathbf{t})\right. \\
\cup \widetilde{\mathcal{Q}}(\mathbf{r}, \mathbf{t}) \cup \overline{\mathscr{P}}(\mathbf{r}, \mathbf{t}) \cup \widetilde{\mathscr{P}}(\mathbf{r}, \mathbf{t})] \subseteq \mathcal{G}\left(\mathbf{r}, \mathcal{X}^{s} \backslash\{\mathbf{r}\}\right) .
\end{aligned}
$$

Hence, we are left to prove the opposite inclusion. In order to do this, we again use the characterization of minimal gates as essential saddles given in [3, Theorem 5.1]. Thus, our strategy is to prove that any

$$
\begin{array}{r}
\eta \in \mathcal{S}\left(\mathbf{r}, \mathcal{X}^{s} \backslash\{\mathbf{r}\}\right) \backslash \bigcup_{\mathbf{t} \in \mathcal{X}^{s} \backslash\{\mathbf{r}\}}\left[\bigcup_{j=2}^{L-3} \mathcal{W}_{j}(\mathbf{r}, \mathbf{t}) \cup \overline{\mathscr{H}}(\mathbf{r}, \mathbf{t}) \cup \widetilde{\mathscr{H}}(\mathbf{r}, \mathbf{t}) \cup \overline{\mathcal{Q}}(\mathbf{r}, \mathbf{t})\right. \\
\cup \widetilde{\mathcal{Q}}(\mathbf{r}, \mathbf{t}) \cup \overline{\mathscr{P}}(\mathbf{r}, \mathbf{t}) \cup \widetilde{\mathscr{P}}(\mathbf{r}, \mathbf{t})]
\end{array}
$$

is an unessential saddle. In particular, for any saddle $\eta$ as in (6.2) and for any $\omega \in \Omega_{\mathbf{r}, \mathcal{X}^{s} \backslash\{\mathbf{r}\}}^{\text {opt }}$ such that $\eta \in \omega$, we prove that conditions (a), (b) of Remark 5.2 are satisfied. Let $\mathbf{s} \in \mathcal{X}^{s} \backslash\{\mathbf{r}\}$ be the first stable state visited by $\omega$. By Lemmas 5.2 and 5.1 the condition (a) of Remark 5.2 is satisfied. Moreover, condition (b) is satisfied by the optimal path $\omega^{\prime} \in \Omega_{\mathbf{r}, \mathcal{X}^{s} \backslash\{\mathbf{r}\}}^{\text {opt }}$ from $\mathbf{r}$ to $\mathbf{s} \in \mathcal{X}^{s} \backslash\{\mathbf{r}\}$ constructed in the proof of Theorem 3.2 since for that path, $\left\{\operatorname{argmax}_{\omega^{\prime}} H\right\} \subseteq$ $\left\{\operatorname{argmax}_{\omega} H\right\} \backslash\{\eta\}$ and $\omega^{\prime} \cap \mathcal{X}^{s} \backslash\{\mathbf{r}, \mathbf{s}\}=\varnothing$. In conclusion, $\eta$ is an unessential saddle.

Proof of Corollary 3.2. The corollary follows by Theorem 3.3 and [3, Theorem 5.4].

\subsection{The Minimal Gates from a Stable State to Another Stable State}

Proof of Theorem 3.5. Let us begin by considering the sets in (a). First, we focus on the sets

$$
\bigcup_{\mathbf{t} \in \mathcal{X}^{s} \backslash\{\mathbf{r}\}} \overline{\mathscr{P}}(\mathbf{r}, \mathbf{t}) \text { and } \bigcup_{\mathbf{t} \in \mathcal{X}^{s} \backslash\{\mathbf{r}\}} \widetilde{\mathscr{P}}(\mathbf{r}, \mathbf{t}) \text {. }
$$

Let $\omega \in \Omega_{\mathbf{r}, \mathbf{s}}^{\text {opt }}$ and let $\mathbf{z} \in \mathcal{X}^{s} \backslash\{\mathbf{r}\}$ be the first stable configuration visited by $\omega$ after $\mathbf{r}$. By Theorem 3.1(a), we get $\omega \cap \overline{\mathscr{P}}(\mathbf{r}, \mathbf{z}) \neq \varnothing$ and $\omega \cap \widetilde{\mathscr{P}}(\mathbf{r}, \mathbf{z}) \neq \varnothing$. Thus,

$$
\omega \cap\left(\bigcup_{\mathbf{t} \in \mathcal{X}^{s} \backslash\{\mathbf{r}\}} \overline{\mathscr{P}}(\mathbf{r}, \mathbf{t})\right) \neq \varnothing \text { and } \omega \cap\left(\bigcup_{\mathbf{t} \in \mathcal{X}^{s} \backslash\{\mathbf{r}\}} \tilde{\mathscr{P}}(\mathbf{r}, \mathbf{t})\right) \neq \varnothing \text {, }
$$


and so these sets are gates. Let us now prove that these sets are in fact minimal gates. There exists $\mathbf{z} \in \mathcal{X}^{s} \backslash\{\mathbf{r}\}$ such that $\eta \in \overline{\mathscr{P}}(\mathbf{r}, \mathbf{z})$. Let $\omega^{(1)}: \mathbf{r} \rightarrow \mathbf{z}$ be the optimal path constructed in the proof of Theorem 3.1(a) such that $\omega^{(1)} \cap \mathcal{X}^{s} \backslash\{\mathbf{r}, \mathbf{z}\}=\varnothing$. Let $\omega^{(2)}: \mathbf{z} \rightarrow \mathbf{s}$ be the reference path given in Definition 5.1. If $\mathbf{z}=\mathbf{s}$, then we set $\omega^{\prime}:=\omega^{(1)}$. Otherwise, we define $\omega^{\prime}$ as the concatenation of the paths $\omega^{(1)}$ and $\omega^{(2)}$. The optimal path associated to $\bigcup_{\mathbf{t} \in \mathcal{X}^{s} \backslash\{\mathbf{r}\}} \widetilde{\mathscr{P}}(\mathbf{r}, \mathbf{t})$ is constructed similarly using the symmetry of the model. In both cases, this concludes the proof that the sets in (6.3) are minimal gates for the transition $\mathbf{r} \rightarrow \mathbf{s}$. Second, let us focus on the sets

$$
\bigcup_{\mathbf{t} \in \mathcal{X}^{s} \backslash\{\mathbf{s}\}} \overline{\mathscr{P}}(\mathbf{t}, \mathbf{s}) \text { and } \bigcup_{\mathbf{t} \in \mathcal{X}^{s} \backslash\{\mathbf{s}\}} \tilde{\mathscr{P}}(\mathbf{t}, \mathbf{s}) .
$$

Again by Theorem 3.1(a) these sets are gates. Let us now prove that they are minimal gates. Let $\mathbf{z} \in \mathcal{X}^{s} \backslash\{\mathbf{s}\}$ such that $\eta \in \overline{\mathscr{P}}(\mathbf{z}, \mathbf{s})$. Let $\omega^{(1)}: \mathbf{r} \rightarrow \mathbf{z}$ be the reference path given in Definition 5.1. Let $\omega^{(2)}: \mathbf{z} \rightarrow \mathbf{s}$ be the path constructed in the proof of Theorem 3.1(a) such that $\omega^{(2)} \cap \mathcal{X}^{s} \backslash\{\mathbf{z}, \mathbf{s}\}=\varnothing$. If $\mathbf{z}=\mathbf{r}$, then we set $\omega^{\prime}:=\omega^{(2)}$. Otherwise, we define $\omega^{\prime}$ as the concatenation of the paths $\omega^{(1)}$ and $\omega^{(2)}$. The optimal path associated to $\bigcup_{\mathbf{t} \in \mathcal{X}^{s} \backslash\{\mathbf{s}\}} \widetilde{\mathscr{P}}(\mathbf{t}, \mathbf{s})$, is constructed similarly. In both cases, (ii) is verified and the sets in (6.5) are minimal gates for the transition $\mathbf{r} \rightarrow \mathbf{s}$. Following the same strategy, we prove that the sets in (b), (c) and (d) are minimal gates for the transition from $\mathbf{r}$ to $\mathbf{s}$ by using Theorem 3.1(b), (c), (d), respectively, instead of Theorem 3.1(a).

Proof of Theorem 3.6. Our aim is to prove that $\mathcal{G}(\mathbf{r}, \mathbf{s})$ only contains the minimal gates of Theorem 3.5. Thus, our strategy is to prove that any

$$
\eta \in \mathcal{S}(\mathbf{r}, \mathbf{s}) \backslash\left(\bigcup_{\mathbf{t} \in \mathcal{X}^{s} \backslash\{\mathbf{r}\}} \mathcal{F}(\mathbf{r}, \mathbf{t}) \cup \bigcup_{\mathbf{t} \in \mathcal{X}^{s} \backslash\{\mathbf{s}\}} \mathcal{F}(\mathbf{t}, \mathbf{s})\right)
$$

does not belong to a minimal gate. To do this, note that

$$
\mathcal{S}(\mathbf{r}, \mathbf{s}) \backslash\left(\bigcup_{\mathbf{t} \in \mathcal{X}^{s} \backslash\{\mathbf{r}\}} \mathcal{F}(\mathbf{r}, \mathbf{t}) \cup \bigcup_{\mathbf{t} \in \mathcal{X}^{s} \backslash\{\mathbf{s}\}} \mathcal{F}(\mathbf{t}, \mathbf{s})\right)=\underset{\substack{\mathbf{t}, \mathbf{z} \in \mathcal{X}^{s} \backslash\{\mathbf{r}, \mathbf{s}\}, \mathbf{t} \neq \mathbf{z}}}{\bigcup} \mathcal{F}(\mathbf{t}, \mathbf{z}) \cup\left(\mathcal{S}(\mathbf{r}, \mathbf{s}) \bigcup_{\substack{\mathbf{t}, \mathbf{z} \in \mathcal{X}^{s}, \mathbf{t} \neq \mathbf{z}}} \mathcal{F}(\mathbf{t}, \mathbf{z})\right) .
$$

The proof of the theorem is given by the following claims

1. $\bigcup_{\mathbf{t}, \mathbf{z} \in \mathcal{X}^{s} \backslash\{\mathbf{r}, \mathbf{s}\}, \mathbf{t} \neq \mathbf{z}} \mathcal{F}(\mathbf{t}, \mathbf{z})$ is not a gate for the transition $\mathbf{r} \rightarrow \mathbf{s}$

2. any $\eta \in \mathcal{S}(\mathbf{r}, \mathbf{s}) \backslash \bigcup_{\mathbf{t}, \mathbf{z} \in \mathcal{X}^{s}, \mathbf{t} \neq \mathbf{z}} \mathcal{F}(\mathbf{t}, \mathbf{z})$ is an ussential saddle.

Indeed, 1. implies in particular that $\bigcup_{\mathbf{t}, \mathbf{z} \in \mathcal{X}^{s} \backslash\{\mathbf{r}, \mathbf{s}\}, \mathbf{t} \neq \mathbf{z}} \mathcal{F}(\mathbf{t}, \mathbf{z})$ is not a minimal gate. On the other hand, 2. implies that any $\eta \in \mathcal{S}(\mathbf{r}, \mathbf{s}) \backslash \bigcup_{\mathbf{t}, \mathbf{z} \in \mathcal{X}^{s}, \mathbf{t} \neq \mathbf{z}} \mathcal{F}(\mathbf{t}, \mathbf{z})$ is not a minimal gate by [3, Theorem 5.1]. In order to prove 1., it is enough to construct an optimal path $\omega \in \Omega_{\mathbf{r}, \mathbf{s}}^{\text {opt }}$ such that $\omega \cap \mathcal{F}(\mathbf{t}, \mathbf{z})=\varnothing$ for any $\mathbf{t}, \mathbf{z} \in \mathcal{X}^{s} \backslash\{\mathbf{r}, \mathbf{s}\}, \mathbf{t} \neq \mathbf{z}$. The reference path $\omega: \mathbf{r} \rightarrow \mathbf{s}$ in Definition 5.1 satisfies these properties, and so 1 . is verified. Let us now prove the claim 2 , i.e., that any $\eta \in \mathcal{S}(\mathbf{r}, \mathbf{s}) \backslash \bigcup_{\mathbf{t}, \mathbf{z} \in \mathcal{X}^{s}, \mathbf{t} \neq \mathbf{z}} \mathcal{F}(\mathbf{t}, \mathbf{z})$ and for any $\omega \in \Omega_{\mathbf{r}, \mathbf{s}}^{\text {opt }}$ such that $\eta \in \omega$ satisfies conditions (a), (b) of Remark 5.2. By Lemmas 5.1 and 5.2 the condition (a) of Remark 5.2 is satisfied. Next, let $\mathbf{t}_{1}, \ldots, \mathbf{t}_{m-1} \in \mathcal{X}^{s} \backslash\{\mathbf{r}, \mathbf{s}\}$ be the stable configurations visited by $\omega$ in $\mathcal{X}^{s} \backslash\{\mathbf{r}, \mathbf{s}\}$ before hitting $\mathbf{s}$. If we set $\mathbf{t}_{0}=\mathbf{r}, \mathbf{t}_{m}=\mathbf{s}$ and $\mathbf{t}_{i} \neq \mathbf{t}_{i+1}$ for all $i=0, \ldots, m-1$, $m \in \mathbb{N}$, we partition the path $\omega$ in the $m$ paths $\omega^{(i)}: \mathbf{t}_{i} \rightarrow \mathbf{t}_{i+1}$ such that their concatenation is $\omega$.

Let $j \in\{0, \ldots, m-1\}$ such that $\eta \in \omega^{(j)}$. We set $\omega^{\prime(j)} \in \Omega_{\mathbf{t}_{j}, \mathbf{t}_{j+1}}^{\text {opt }}$ the optimal path constructed to prove condition (b) of Remark 5.2 in Theorem 3.2 such that 
$\left\{\operatorname{argmax}_{\omega^{\prime(j)}} H\right\} \subseteq\left\{\operatorname{argmax}_{\omega^{(j)}} H\right\} \backslash\{\eta\}$ and $\omega^{\prime(j)} \cap \mathcal{X}^{s} \backslash\left\{\mathbf{t}_{j}, \mathbf{t}_{j+1}\right\}=\varnothing$. Thus we define a path $\omega^{\prime}$ as the concatenation of the $m$ paths $\omega^{(1)}, \ldots, \omega^{(j-1)}, \ldots, \omega^{(j)}, \omega^{(j+1)}, \ldots, \omega^{(m)}$ such that $\left\{\operatorname{argmax}_{\omega^{\prime}} H\right\} \subseteq\left\{\operatorname{argmax}_{\omega} H\right\} \backslash\{\eta\}$. Hence, both the conditions of Remark 5.2 are satisfied and $\eta$ is an unessential saddle.

Proof of Theorem 3.6. Since Theorem 3.5 holds, the corollary follows by [3, Theorem 5.4].

\section{Restricted-Tube and Tube of Typical Paths: Proofs}

In this section we prove the main results on the restricted-tube of typical paths and on the tube of typical paths stated in Sect. 4.2.

Remark 7.1 Given a $q$-Potts configuration $\sigma \in \mathcal{X}$ on a grid-graph $\Lambda$, a vertex $v \in V$ and a spin value $s \in\{1, \ldots, q\}$ such that $\sigma(v) \neq s$, using (2.6) we have

$$
H(\sigma)-H\left(\sigma^{v, s}\right) \in\{-4,-2,0,2,4\} .
$$

It follows that the principal boundary of extended cycles $\mathcal{C}$ in (4.14)-(4.18) is the union of those configurations $\bar{\sigma} \in \partial \mathcal{C}$ such that either

(i) $H(\bar{\sigma})-H(\sigma)=-2$, or

(ii) $H(\bar{\sigma})-H(\sigma)=-4$.

Let us now state some useful lemmas which we prove at the end this section by using Remark 7.1. It is understood that all results hold for the $q$-state Potts model on a $K \times L$ grid $\Lambda$ with periodic boundary conditions.

Lemma 7.1 Let $\mathbf{r}, \mathbf{s} \in \mathcal{X}^{s}, \mathbf{r} \neq \mathbf{s}$. Then,

$$
\mathcal{B}(\overline{\mathcal{K}}(r, s))=\overline{\mathcal{D}}_{1}(r, s) \cup \overline{\mathcal{D}}_{2}(r, s) \cup \overline{\mathcal{E}}_{1}(r, s) \cup \overline{\mathcal{E}}_{2}(r, s) .
$$

The principal boundary (7.2) is illustrated in the first row of Fig. 7.

Lemma 7.2 Let $\mathbf{r}, \mathbf{s} \in \mathcal{X}^{s}, \mathbf{r} \neq \mathbf{s}$. Then, for any $i=1, \ldots, K-4$,

$$
\begin{aligned}
& \mathcal{B}\left(\overline{\mathcal{D}}_{i}(r, s)\right)=\overline{\mathcal{D}}_{i+1}(r, s) \cup \overline{\mathcal{D}}_{i+2}(r, s) \cup \overline{\mathcal{E}}_{i+1}(r, s) \cup \overline{\mathcal{E}}_{i+2}(r, s), \\
& \mathcal{B}\left(\overline{\mathcal{E}}_{i}(r, s)\right)=\overline{\mathcal{E}}_{i+1}(r, s) \cup \overline{\mathcal{E}}_{i+2}(r, s) .
\end{aligned}
$$

The principal boundaries (7.3)-7.4 are illustrated in the middle rows of Fig. 7.

Lemma 7.3 Let $\mathbf{r}, \mathbf{s} \in \mathcal{X}^{s}, \mathbf{r} \neq \mathbf{s}$. Then,

$$
\begin{aligned}
& \mathcal{B}\left(\overline{\mathcal{D}}_{K-3}(r, s)\right)=\overline{\mathcal{D}}_{K-2}(r, s) \cup \overline{\mathcal{E}}_{K-2}(r, s) \cup \bar{R}_{1,1}(\mathbf{r}, \mathbf{s}), \\
& \mathcal{B}\left(\overline{\mathcal{E}}_{K-3}(r, s)\right)=\overline{\mathcal{E}}_{K-2}(r, s) \cup \bar{R}_{1,1}(r, s), \\
& \mathcal{B}\left(\overline{\mathcal{D}}_{K-2}(r, s)\right)=\mathcal{B}\left(\overline{\mathcal{E}}_{K-2}(r, s)\right)=\bar{R}_{1,1}(r, s) .
\end{aligned}
$$

The principal boundaries described in Lemma 7.3 are illustrated in the last rows of Fig. 7.

Proof of Theorem 4.1. We will prove that $\mathscr{U}_{\mathbf{s}}(\mathbf{r})$ in (4.19) contains $\mathcal{C} \in \mathcal{M}\left(\mathcal{C}_{\mathbf{s}}^{+}(\mathbf{r}) \backslash \mathbf{s}\right)$ that belong to at least a cycle-path $\left(\mathcal{C}_{1}, \ldots, \mathcal{C}_{n}\right) \in J_{\mathbf{r}, \mathbf{s}}, n \in \mathbb{N}$, such that $\bigcup_{i=1}^{n} \mathcal{C}_{i} \cap \mathcal{X}^{s} \backslash\{\mathbf{r}, \mathbf{s}\}=\varnothing$, $\mathcal{C}_{1}=\mathcal{C}_{\mathbf{s}}(\mathbf{r})$ and $\mathbf{s} \in \partial \mathcal{C}_{n}$, see 4.1.1. 
We start by studying the first descent from a trivial cycle $\left\{\xi^{*}\right\}$ for some $\xi^{*} \in \bar{R}_{\left\lfloor\frac{L}{2}\right\rfloor, K}(r, s)$ to $\mathbf{r}$, where $\lfloor n\rfloor:=\max \{m \in \mathbb{Z}: m \leq n\}$. Using the symmetry of the model on $\Lambda$, we describe similarly the first descent from the same configuration $\xi^{*}$ to s. Finally using reversibility, we will obtain a complete description of $\mathscr{U}_{\mathbf{s}}(\mathbf{r})$ by joining the time reversal of the first descent from $\left\{\xi^{*}\right\}$ to $\mathbf{r}$ with the first discent from $\left\{\xi^{*}\right\}$ to $\mathbf{s}$.

For the sake of semplicity, we separate the description of the first descent from $\left\{\xi^{*}\right\}$ to $\mathbf{r}$ for some $\xi^{*} \in \bar{R}_{\left\lfloor\frac{L}{2}\right\rfloor, K}(r, s)$ in several steps. We start by studying the typical trajectories followed by the process during the transition from $\left\{\xi^{*}\right\}$ to $\bar{R}_{2, K}(\mathbf{r}, \mathbf{s}) \subset \partial \overline{\mathcal{K}}(r, s)$, see (4.14), and then we study the typical paths followed for the first descent from $\overline{\mathcal{K}}(r, s)$ to $\mathbf{r}$. Note that $\partial \mathcal{C}_{\mathbf{s}}(\mathbf{r}) \cap \overline{\mathcal{K}}(r, s) \neq \varnothing$. Using Lemma 5.2(a) and (c), for any $i=\left\lfloor\frac{L}{2}\right\rfloor-1, \ldots, 2$ we define a cycle-path $\left(\mathcal{C}_{i}^{0}, \mathcal{C}_{i}^{1}, \mathcal{C}_{i}^{2}\right)$ such that

$-\mathcal{C}_{i}^{0}=\left\{\eta_{K}\right\}$ for $\eta_{K} \in \bar{R}_{i+1, K}$,

$-\mathcal{C}_{i}^{1}=\bigcup_{j=1}^{K-1}\left\{\eta_{j}\right\}$ for $\eta_{j} \in \bar{B}_{i, K}^{j}$,

$-\mathcal{C}_{i}^{2}=\left\{\eta_{0}\right\}$ for $\eta_{0} \in \bar{R}_{i, K}$,

where $\eta_{i}:=\eta_{i+1}^{v, r}$ for some $v=v(i) \in V$. Note that $\mathcal{C}_{i}^{0}, \mathcal{C}_{i}^{2}$ are non-trivial cycles. For any $i=\left\lfloor\frac{L}{2}\right\rfloor-1, \ldots, 2$, using Lemma 5.2, we also remark that for any $\sigma \in \bar{R}_{i, K}, \mathscr{F}(\partial\{\sigma\}) \subset$ $\bar{B}_{i-1, K}^{K-1} \cup \bar{B}_{i, K}^{1}$. Then, using Lemma 5.1 we have that $\{\sigma\}$ satisfies (4.2) and that

$$
\mathcal{B}(\{\sigma\})=\mathscr{F}(\partial\{\sigma\}) .
$$

Moreover, using Lemmas 5.1 and 5.2 we remark that for any $i=\left\lfloor\frac{L}{2}\right\rfloor-1, \ldots, 2, \mathcal{C}_{i}^{1}$ is a plateau and its principal boundary is given by

$$
\mathcal{B}\left(\mathcal{C}_{i}^{1}\right)=\mathcal{C}_{i}^{0} \cup \mathcal{C}_{i}^{2}
$$

Thus, $\left(\mathcal{C}_{i}^{0}, \mathcal{C}_{i}^{1}, \mathcal{C}_{i}^{2}\right) \in J_{\mathcal{C}_{i}^{0}, \mathcal{C}_{i}^{2}}$ since (4.6) is satisfied. Hence, starting from $\mathcal{C}_{\left\lfloor\frac{L}{2}\right\rfloor}^{0}=\left\{\xi^{*}\right\}$ for some $\xi^{*} \in \bar{R}_{\left\lfloor\frac{L}{2}\right\rfloor, K}(r, s)$, we depict a cycle-path vtj-connected to $\mathcal{C}_{2}^{2}=\{\hat{\eta}\}$ for some appropriate $\hat{\eta} \in \bar{R}_{2, K}$ as

$$
\left(\mathcal{C}_{\left\lfloor\frac{L}{2}\right\rfloor}^{0}, \mathcal{C}_{\left\lfloor\frac{L}{2}\right\rfloor-1}^{1}, \mathcal{C}_{\left\lfloor\frac{L}{2}\right\rfloor-1}^{2} \equiv \mathcal{C}_{\left\lfloor\frac{L}{2}\right\rfloor-1}^{0}, \ldots, \mathcal{C}_{3}^{2} \equiv \mathcal{C}_{2}^{0}, \mathcal{C}_{2}^{1}, \mathcal{C}_{2}^{2}\right)
$$

Let us now describe a cycle-path

$$
\left(\overline{\mathcal{C}}_{1}, \ldots, \overline{\mathcal{C}}_{m}\right)
$$

which is vtj-connected to $\mathbf{r}$ and such that $\overline{\mathcal{C}}_{1}=\left\{\bar{\eta}^{*}\right\}$, with $\bar{\eta}^{*} \in \overline{\mathscr{P}}(\mathbf{r}, \mathbf{s})$. This cycle-path exists in view of Lemma 5.4 and it is chosen in such a way that it is defined by a spin update in a vertex of $\hat{\eta}$. Note that any set from (4.14) to (4.18) is an extended cycle, i.e., a maximal connected set of equielevated trivial cycles. Thanks to Lemmas 7.1, 7.2 and 7.3 we obtain that for every $i=m, \ldots, n-1$, the cycle-path (7.9) is characterized by a sequence of cycles and extended cycles $\overline{\mathcal{C}}_{1}, \ldots, \overline{\mathcal{C}}_{m}$ such that

$$
\overline{\mathcal{C}}_{1}, \ldots, \overline{\mathcal{C}}_{m} \subset \overline{\mathcal{K}}(r, s) \cup \bigcup_{i=1}^{K-2}\left(\overline{\mathcal{D}}_{i}(r, s) \cup \overline{\mathcal{E}}_{i}(r, s)\right) \cup \bar{R}_{1,1}(r, s)
$$

and $\overline{\mathcal{C}}_{m}=$ r. More precisely, $\left(\overline{\mathcal{C}}_{1}, \ldots, \overline{\mathcal{C}}_{m}\right) \in J_{\overline{\mathcal{C}}_{1}, \overline{\mathcal{C}}_{m}}$. Indeed, using Lemmas 7.1, 7.2 and 7.3 it follows that for any pair of consecutive cycles the condition (4.6) is satisfied. 
Arguing similarly, we construct a cycle-path vtj-connected from $\left\{\xi^{*}\right\}$ to $\mathbf{s}$. Indeed, first we construct the following

$$
\left(\mathcal{C}_{\left\lfloor\frac{L}{2}\right\rfloor}^{0}, \mathcal{C}_{\left\lfloor\frac{L}{2}\right\rfloor+1}^{1}, \mathcal{C}_{\left\lfloor\frac{L}{2}\right\rfloor+1}^{2} \equiv \mathcal{C}_{\left\lfloor\frac{L}{2}\right\rfloor+1}^{0}, \ldots, \mathcal{C}_{L-3}^{2} \equiv \mathcal{C}_{L-2}^{0}, \mathcal{C}_{L-2}^{1}, \mathcal{C}_{L-2}^{2}\right) \in J_{\left\{\xi^{*}\right\},\{\check{\eta}\}}
$$

from $\left\{\xi^{*}\right\}$ to $\{\check{\eta}\}$ for some $\check{\eta} \in \bar{R}_{L-2, K}(r, s)$. Then, we define

$$
\left(\tilde{\mathcal{C}}_{1}, \ldots, \tilde{\mathcal{C}}_{m}\right) \in J_{\tilde{\mathcal{C}}_{1}, \tilde{\mathcal{C}}_{m}}
$$

for the first descent from $\left\{\tilde{\eta}^{*}\right\}$ to $\mathbf{s}$, where $\tilde{\eta}^{*} \in \widetilde{\mathscr{P}}(\mathbf{r}, \mathbf{s})$ exists in view of Lemma 5.4 and it is chosen in such a way that it is defined by a spin update in a vertex of $\check{\eta}$. More precisely, using Lemma 5.2(b) and (d), for any $i=\left\lfloor\frac{L}{2}\right\rfloor+1, \ldots, L-2$ we define a cycle-path $\left(\mathcal{C}_{i}^{0}, \mathcal{C}_{i}^{1}, \mathcal{C}_{i}^{2}\right)$ such that

$-\mathcal{C}_{i}^{0}=\left\{\eta_{0}\right\}$ for $\eta_{0} \in \bar{R}_{i-1, K}$,

$-\mathcal{C}_{i}^{1}=\bigcup_{j=1}^{K-1}\left\{\eta_{j}\right\}$ for $\eta_{j} \in \bar{B}_{i, K}^{j}$,

- $\mathcal{C}_{i}^{2}=\left\{\eta_{K}\right\}$ for $\eta_{K} \in \bar{R}_{i, K}$,

where $\eta_{i}:=\eta_{i-1}^{v, r}$ for some $v=v(i) \in V$. On the other hand, thanks to the symmetry of the model on $\Lambda$, we prove the analogue of Lemmas 7.1, 7.2 and 7.3 for $\widetilde{\mathcal{K}}(r, s), \widetilde{\mathcal{D}}_{i}(r, s), \widetilde{\mathcal{E}}_{i}(r, s)$ for any $i=1, \ldots, K-2$. Thus, we construct the cycle-path vtj-connected $\left(\tilde{\mathcal{C}}_{1}, \ldots, \tilde{\mathcal{C}}_{m}\right)$ from $\left\{\tilde{\eta}^{*}\right\}$ to $\mathbf{s}$ with

$$
\tilde{\mathcal{C}}_{1}, \ldots, \tilde{\mathcal{C}}_{m} \subset \tilde{\mathcal{K}}(r, s) \cup \bigcup_{i=1}^{K-2}\left(\widetilde{\mathcal{D}}_{i}(r, s) \cup \tilde{\mathcal{E}}_{i}(r, s)\right) \cup \tilde{R}_{1,1}(r, s) .
$$

Finally, using reversibility and in view of the above construction, we construct a cycle-path vtj-connected from $\mathbf{r}$ to $\mathbf{s}$ as

$$
\begin{gathered}
\left(\overline{\mathcal{C}}_{m} \equiv \mathbf{r}, \ldots, \overline{\mathcal{C}}_{1}, \mathcal{C}_{2}^{2}, \mathcal{C}_{2}^{1}, \mathcal{C}_{3}^{2} \equiv \mathcal{C}_{2}^{0}, \ldots, \mathcal{C}_{\left\lfloor\frac{L}{2}\right\rfloor-1}^{2} \equiv \mathcal{C}_{\left\lfloor\frac{L}{2}\right\rfloor-1}^{0}, \mathcal{C}_{\left\lfloor\frac{L}{2}\right\rfloor-1}^{1}, \mathcal{C}_{\left\lfloor\frac{L}{2}\right\rfloor}^{0} \equiv\left\{\xi^{*}\right\}\right. \\
\left.\mathcal{C}_{\left\lfloor\frac{L}{2}\right\rfloor+1}^{1}, \mathcal{C}_{\left\lfloor\frac{L}{2}\right\rfloor+1}^{2} \equiv \mathcal{C}_{\left\lfloor\frac{L}{2}\right\rfloor+1}^{0}, \ldots, \mathcal{C}_{L-3}^{2} \equiv \mathcal{C}_{L-2}^{0}, \mathcal{C}_{L-2}^{1}, \mathcal{C}_{L-2}^{2}, \tilde{\mathcal{C}}_{1}, \ldots, \tilde{\mathcal{C}}_{m} \equiv \mathbf{s}\right) .
\end{gathered}
$$

Using (7.13), we obtain that the restricted-tube of typical paths between $\mathbf{r}$ and $\mathbf{s}$ is (4.19). Finally, (4.20) follows by [12, Lemma 3.13].

We are now ready to prove Theorems 4.2 and 4.3.

Proof of Theorem 4.2. Using the description of the restricted-tube of typical paths between two stable states, we depict the tube $\mathfrak{T}_{\mathcal{X}^{s} \backslash\{\mathbf{r}\}}(\mathbf{r})$ as in (4.21). Indeed, any cycle-path $\left(\mathcal{C}_{1}, \ldots, \mathcal{C}_{n}\right), n \in \mathbb{N}$, vtj-connected to $\mathcal{X}^{s} \backslash\{\mathbf{r}\}$ starting from $\mathbf{r}$ is described by a sequence of cycles belonging to some $\mathscr{U}_{\mathbf{s}}(\mathbf{r})$ where $\mathbf{s}$ is the first stable configuration visited by that $\left(\mathcal{C}_{1}, \ldots, \mathcal{C}_{n}\right)$ in $\mathcal{X}^{s} \backslash\{\mathbf{r}\}$. Finally, (4.22) follows by [12, Lemma 3.13].

Proof of Theorem 4.3. Starting from $\mathbf{r}$, the process may intersect some stable configurations different from the target $\mathbf{s}$. It follows that, when we study the typical trajectories for the transition $\mathbf{r} \rightarrow \mathbf{s}$ we have to take into account the possible intermediate transitions between two stable states and such that do not intersect other stable configurations beyond them. For any of these intermediate transitions, we apply the result of the restricted-case. It follows that (4.23) is the tube of typical trajectories. Moreover, (4.24) follows by [12, Lemma 3.13].

Proof of Lemmas 7.1, 7.2, 7.3 


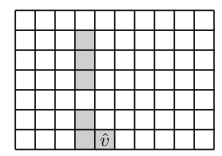

(i) $\bar{\sigma}:=\sigma^{\hat{v}, r} \in \overline{\mathcal{E}}_{1}(r, s)$

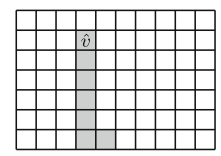

(ii) $\bar{\sigma}:=\sigma^{\hat{v}, r} \in \overline{\mathcal{D}}_{1}(r, s)$

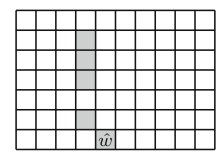

(iii) $\bar{\sigma}:=\sigma^{\hat{w}, r} \in \overline{\mathcal{E}}_{2}(r, s)$

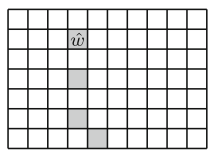

(iv) $\bar{\sigma}:=\sigma^{\hat{w}, r} \in \overline{\mathcal{D}}_{2}(r, s)$

Fig. 13 Examples of $\sigma \in \overline{\mathcal{K}}(r, s)$ and $\bar{\sigma} \in \mathcal{B}(\overline{\mathcal{K}}(r, s)$. We color white the vertices with spin $r$ and gray those vertices whose spin is $s$

Proof of Lemma 7.1 According to (4.3), we describe the principal boundary of the extended cycle $\overline{\mathcal{K}}(r, s)$ by looking for those configurations $\bar{\sigma} \notin \overline{\mathcal{K}}(r, s)$ which communicate with some $\sigma \in \overline{\mathcal{K}}(r, s)$ such that $\sigma$ and $\bar{\sigma}$ satisfy either case (i) or case (ii) of Remark 7.1. Let us start to consider (i) of Remark 7.1. In view of (4.14), this case occurs only when $\sigma$ has a spin $s$ with three nearest-neighbor spins $r$ and $\bar{\sigma}$ is obtained from $\sigma$ by flipping from $s$ to $r$ this spin $s$. In particular, we note that $\sigma \in \overline{\mathcal{K}}(r, s)$ has a spin $s$ with three nearest-neighbor spins $r$ only when $\sigma \in \overline{\mathcal{K}}(r, s) \backslash[\overline{\mathcal{Q}}(\mathbf{r}, \mathbf{s}) \cup \overline{\mathscr{P}}(\mathbf{r}, \mathbf{s})]$, i.e., when

$$
\begin{gathered}
\sigma \in \overline{\mathscr{H}}(\mathbf{r}, \mathbf{s}) \cup\{\sigma \in \mathcal{X}: \sigma(v) \in\{r, s\} \forall v \in V, H(\sigma)=2 K+2+H(\mathbf{r}), \sigma \text { has at } \\
\text { least two } \left.s \text {-interacting clusters, and } R\left(\mathrm{C}^{s}(\sigma)\right)=R_{2 \times(K-1)}\right\} .
\end{gathered}
$$

Hence, consider $\sigma \in \overline{\mathcal{K}}(r, s)$ with a spin $s$, say on vertex $\hat{v}$, with three nearest-neighbor spins $r$ and one nearest-neighbor spin $s$ and define $\bar{\sigma}:=\sigma^{\hat{v}, r}$. We note that

- for any $h=2, \ldots, K-2$, if $\sigma \in \bar{B}_{1, K-1}^{h}(r, s)$, then $\bar{\sigma} \in \bar{B}_{1, K-1}^{h-1}(r, s) \subset \overline{\mathcal{D}}_{1}(r, s)$;

- if $\sigma \in \bar{B}_{1, K}^{1}(r, s)$, then $\bar{\sigma} \in \bar{R}_{1, K}(r, s) \subset \overline{\mathcal{E}}_{1}(r, s)$;

- if $\hat{v}$ has its unique nearest-neighbor spin $s$ on an adjacent column, then $\bar{\sigma} \in \overline{\mathcal{E}}_{1}(r, s)$, see Fig. 13i;

- if $\hat{v}$ and its nearest-neighbor spin $s$ lie on the same column, then $\bar{\sigma} \in \overline{\mathcal{D}}_{1}(r, s)$, see Fig. 13 ii.

Next we move to consider (ii) of Remark 7.1. This occurs only when $\sigma$ has a spin $s$, say on vertex $\hat{w}$, sourrounded by four spins $r$ and $\bar{\sigma}:=\sigma^{\hat{w}, r}$. This happens when $\sigma \in$ $\overline{\mathcal{K}}(r, s) \backslash[\overline{\mathscr{H}}(\mathbf{r}, \mathbf{s}) \cup \overline{\mathcal{Q}}(\mathbf{r}, \mathbf{s}) \cup \mathscr{P}(\mathbf{r}, \mathbf{s})]$. Then, we note that

- if $R\left(\mathrm{C}^{s}(\bar{\sigma})\right)=R_{1 \times(K-2)}$, i.e., if $\hat{w}$ lies on a column where there are no other spins $s$, then $\bar{\sigma} \in \overline{\mathcal{E}}_{2}(r, s)$, see Fig. 13iii;

- if $R\left(\mathrm{C}^{s}(\bar{\sigma})\right)=R_{2 \times(K-3)}$, i.e., if $\hat{w}$ lies on a column where there are other spins $s$, then $\bar{\sigma} \in \overline{\mathcal{D}}_{2}(r, s)$, see Fig. 13iv.

Proof of Lemma 7.2 Similarly to the proof of Lemma 7.1, for any $i=1, \ldots, K-4$ we describe the principal boundary of the extended cycles $\overline{\mathcal{D}}_{i}(r, s)$ and $\overline{\mathcal{E}}_{i}(r, s)$ by using Remark 7.1. For any $i=1, \ldots, K-4$, let us start by studying the principal boundary of $\overline{\mathcal{D}}_{i}(r, s)$. Case Remark 7.1(i) occurs only when $\sigma \in \overline{\mathcal{D}}_{i}(r, s)$ has a spin $s$, say on vertex $\hat{v}$ with three nearest-neighbor spins $r$ and one nearest-neighbor spin $s$ and $\bar{\sigma}:=\sigma^{\hat{v}, r}$. Then, we note that

- if $\hat{v}$ its unique nearest-neighbor spin $s$ on an adjacent column, then $R\left(C^{s}(\bar{\sigma})\right)=R_{1 \times(K-(i+1))}$ and $\bar{\sigma} \in \overline{\mathcal{E}}_{i+1}(r, s)$, see Fig. 14ii;

- if $\hat{v}$ and its nearest-neighbor spin $s$ lie on the same column, then $R\left(\mathrm{C}^{s}(\bar{\sigma})\right)=R_{2 \times(K-(i+2))}$ and $\bar{\sigma} \in \overline{\mathcal{D}}_{i+1}(r, s)$, see Fig. 14i.

Regarding (ii) of Remark 7.1, it occurs only when $\sigma$ has a spin $s$, say on vertex $\hat{w}$, sourrounded by four spins $r$ and $\bar{\sigma}:=\sigma^{\hat{w}, r}$. It follows that 


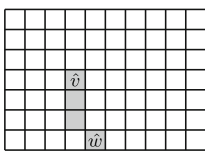

(i) $\bar{\sigma}:=\sigma^{\hat{v}, r} \in \overline{\mathcal{D}}_{3}(r, s)$ $\bar{\sigma}:=\sigma^{\hat{w}, r} \in \overline{\mathcal{E}}_{4}(r, s)$

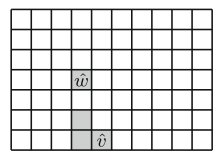

(ii) $\bar{\sigma}:=\sigma^{\hat{v}, r} \in \overline{\mathcal{E}}_{3}(r, s)$ $\bar{\sigma}:=\sigma^{\hat{w}, r} \in \overline{\mathcal{D}}_{4}(r, s)$

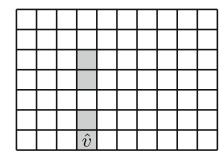

(iv) $\bar{\sigma}:=\sigma^{\hat{v}, r} \in \underline{\mathcal{E}}_{3}(r, s)$

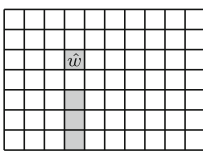

(iii) $\bar{\sigma}:=\sigma^{\hat{w}, r} \in \underline{\overline{\mathcal{E}}}_{4}(r, s)$

Fig. 14 Examples of $\sigma \in \overline{\mathcal{D}}_{2}(r, s)$ and $\bar{\sigma} \in \mathcal{B}\left(\overline{\mathcal{D}}_{2}(r, s)\right)$ in (i) and (ii); examples of $\sigma \in \overline{\mathcal{E}}_{2}(r, s)$ and $\bar{\sigma} \in \mathcal{B}\left(\overline{\mathcal{E}}_{2}(r, s)\right)$ in (iii) and (iv). We color white the vertices with spin $r$ and gray those vertices whose spin is $s$

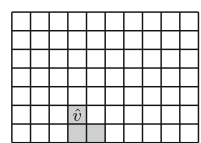

(i) $\bar{\sigma}:=\sigma^{\hat{v}, r} \in \overline{\mathcal{D}}_{K-2}(r, s)$

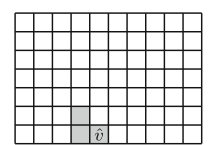

(ii) $\bar{\sigma}:=\sigma^{\hat{v}, r} \in \overline{\mathcal{E}}_{K-2}(r, s)$

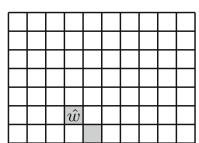

(iii) $\bar{\sigma}:=\sigma^{\hat{w}, r} \in \bar{R}_{1,1}(r, s)$

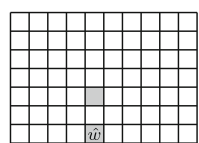

(iv) $\bar{\sigma}:=\sigma^{\hat{w}, r} \in \bar{R}_{1,1}(r, s)$

Fig. 15 Examples of $\sigma \in \overline{\mathcal{D}}_{K-3}(r, s)$ and $\bar{\sigma} \in \mathcal{B}\left(\overline{\mathcal{D}}_{K-3}(r, s)\right)$. We color white the vertices with spin $r$ and gray those vertices whose spin is $s$

- if $R\left(\mathrm{C}^{s}(\bar{\sigma})\right)=R_{1 \times(K-(i+2))}$, i.e., if $\hat{w}$ lies on a column where there are no other spins $s$, then $\bar{\sigma} \in \overline{\mathcal{E}}_{i+2}(r, s)$, see Fig. $14 \mathrm{i}$;

- if $R\left(\mathrm{C}^{s}(\bar{\sigma})\right)=R_{2 \times(K-(i+3))}$, i.e., if $\hat{w}$ lies on a column where there are other spins $s$, then $\bar{\sigma} \in \overline{\mathcal{D}}_{i+2}(r, s)$, see Fig. 14ii.

Next we move to describe the principal boundary of $\overline{\mathcal{E}}_{i}(r, s)$ for any $i=1, \ldots, K-4$. Case (i) of Remark 7.1 occurs when $\sigma \in \overline{\mathcal{E}}_{i}(r, s)$ has a spin $s$, say on vertex $\hat{v}$, with three nearestneighbor spins $r$ and one nearest-neighbor spin $s$ and $\bar{\sigma}:=\sigma^{\hat{v}, r}$. Hence, $\bar{\sigma} \in \overline{\mathcal{E}}_{i+1}(r, s)$, see Fig. 14ii. On the other hand, case (ii) of Remark 7.1 occurs only when $\sigma$ has a spin $s$, say on vertex $\hat{w}$, sourrounded by four spins $r$ and $\bar{\sigma}:=\sigma^{\hat{w}, r}$. Thus $\bar{\sigma} \in \overline{\mathcal{E}}_{i+2}(r, s)$, see Fig. 14iv.

Proof of Lemma 7.3 First, note that from (4.17) and (4.18) we have

$$
\overline{\mathcal{D}}_{K-2}(r, s)=\bar{R}_{2,1}(r, s) \text { and } \overline{\mathcal{E}}_{K-2}(r, s)=\bar{R}_{1,2}(r, s) .
$$

Once again we describe the principal boundary of the extended cycles $\overline{\mathcal{D}}_{i}(r, s)$ and $\overline{\mathcal{E}}_{i}(r, s)$ for $i=K-3, K-2$ by using Remark 7.1. Let us begin by studying the principal boundary of $\overline{\mathcal{D}}_{K-3}(r, s)$. Case (i) of Remark 7.1 takes place if $\sigma$ has a spin $s$, say on vertex $\hat{v}$, with three nearest-neighbor spins $r$ and one nearest-neighbor $s$ and $\bar{\sigma}:=\sigma^{\hat{v}, r}$. Hence, it occurs only when $\sigma \in \bar{B}_{1,2}^{1}(r, s)$ and either $\bar{\sigma} \in \overline{\mathcal{D}}_{K-2}(r, s)$ or $\bar{\sigma} \in \overline{\mathcal{E}}_{K-2}(r, s)$, see Fig. 15 i and ii. Case (ii) of Remark 7.1 occurs when $\sigma$ has only two spins $s$ and they lie on the diagonal of a rectangle $R_{2 \times 2}$, i.e., when $\bar{\sigma}$ is obtained by flipping from $s$ to $r$ one of these two spins $s$ and $\bar{\sigma} \in \bar{R}_{1,1}(r, s)$, see Fig. 15iii.

Next we describe the principal boundary of $\overline{\mathcal{E}}_{K-3}(r, s)$. Case (ii) of Remark 7.1 occurs when $\sigma \in \overline{\mathcal{E}}_{K-3}(r, s)$ has a spin $s$, say on vertex $\hat{v}$, with three nearest-neighbor spins $r$ and one nearest-neighbor $s$ and $\bar{\sigma}:=\sigma^{\hat{v}, r}$. Hence, when $\sigma \in \bar{R}_{1,3}(r, s)$ and $\bar{\sigma} \in \overline{\mathcal{E}}_{K-2}(r, s)$. Finally, case (ii) of Remark 7.1 is verified when $\sigma \in \overline{\mathcal{E}}_{K-3}(r, s)$ has two spins $s$ with four nearest-neighbor spins $r$ and one of them is flipped to $r$, i.e., when $\bar{\sigma} \in \bar{R}_{1,1}(r, s)$, see Fig. 15iv. To conclude, we show that (7.7) follows by Remark 7.1 and (7.14). Indeed, $\overline{\mathcal{D}}_{K-2}(r, s)$ and $\overline{\mathcal{E}}_{K-2}(r, s)$ contain those configurations in which there are two spins $s$ with three nearestneighbor spins $r$ and by flipping from $s$ to $r$ one of them we obtain a configuration belonging to their principal boundary. 
Funding Open access funding provided by Università degli Studi di Firenze within the CRUI-CARE Agreement.

Open Access This article is licensed under a Creative Commons Attribution 4.0 International License, which permits use, sharing, adaptation, distribution and reproduction in any medium or format, as long as you give appropriate credit to the original author(s) and the source, provide a link to the Creative Commons licence, and indicate if changes were made. The images or other third party material in this article are included in the article's Creative Commons licence, unless indicated otherwise in a credit line to the material. If material is not included in the article's Creative Commons licence and your intended use is not permitted by statutory regulation or exceeds the permitted use, you will need to obtain permission directly from the copyright holder. To view a copy of this licence, visit http://creativecommons.org/licenses/by/4.0/.

\section{References}

1. Nardi, F.R., Zocca, A.: Tunneling behavior of Ising and Potts models in the low-temperature regime. Stochast. Process. Appl. 129(11), 4556-4575 (2019)

2. Kim, S., Seo, I.: Metastability of stochastic Ising and Potts models on lattices without external fields. arXiv:2102.05565, (2021)

3. Manzo, F., Nardi, F.R., Olivieri, E., Scoppola, E.: On the essential features of metastability: tunnelling time and critical configurations. J. Stat. Phys. 115(1-2), 591-642 (2004)

4. Bet, G., Gallo, A., Nardi, F.R.: Metastability for the degenerate potts model with non-zero external magnetic field under glauber dynamics. arXiv preprint arXiv:2105.14335, (2021)

5. Cassandro, M., Galves, A., Olivieri, E., Vares, M.E.: Metastable behavior of stochastic dynamics: a pathwise approach. J. Stat. Phys. 35(5), 603-634 (1984)

6. Olivieri, E., Scoppola, E.: Markov chains with exponentially small transition probabilities: first exit problem from a general domain. I. The reversible case. J. Stat. Phys. 79(3), 613-647 (1995)

7. Olivieri, E., Scoppola, E.: Markov chains with exponentially small transition probabilities: first exit problem from a general domain. II. The general case. J. Stat. Phys. 84(5), 987-1041 (1996)

8. Olivieri, E., Vares, M.E.: Large Deviations and Metastability, vol. 100. Cambridge University Press, Cambridge (2005)

9. Catoni, O., Cerf, R.: The exit path of a Markov chain with rare transitions. ESAIM 1, 95-144 (1997)

10. Cirillo, E.N., Nardi, F.R.: Relaxation height in energy landscapes: an application to multiple metastable states. J. Stat. Phys. 150(6), 1080-1114 (2013)

11. Cirillo, E.N., Nardi, F.R., Sohier, J.: Metastability for general dynamics with rare transitions: escape time and critical configurations. J. Stat. Phys. 161(2), 365-403 (2015)

12. Nardi, F.R., Zocca, A., Borst, S.C.: Hitting time asymptotics for hard-core interactions on grids. J. Stat. Phys. 162(2), 522-576 (2016)

13. Fernandez, R., Manzo, F., Nardi, F., Scoppola, E., et al.: Asymptotically exponential hitting times and metastability: a pathwise approach without reversibility. Electron. J. Probab. 20, 1-37 (2015)

14. Fernandez, R., Manzo, F., Nardi, F., Scoppola, E., Sohier, J., et al.: Conditioned, quasi-stationary, restricted measures and escape from metastable states. Ann. Appl. Prob. 26(2), 760-793 (2016)

15. Arous, G.B., Cerf, R., et al.: Metastability of the three dimensional Ising model on a torus at very low temperatures. Electron. J. Probab. 1, 1-99 (1996)

16. Cirillo, E.N., Lebowitz, J.L.: Metastability in the two-dimensional Ising model with free boundary conditions. J. Stat. Phys. 90(1), 211-226 (1998)

17. Cirillo, E.N., Olivieri, E.: Metastability and nucleation for the Blume-Capel model. Different mechanisms of transition. J. Stat. Phys. 83(3), 473-554 (1996)

18. Koteckỳ, R., Olivieri, E.: Shapes of growing droplets: a model of escape from a metastable phase. J. Stat. Phys. 75(3), 409-506 (1994)

19. Nardi, F.R., Olivieri, E.: Low temperature stochastic dynamics for an Ising model with alternating field. Markov Proc. Relat. Fields 2, 117-166 (1996)

20. Neves, E.J., Schonmann, R.H.: Critical droplets and metastability for a glauber dynamics at very low temperatures. Commun. Math. Phys. 137(2), 209-230 (1991)

21. Neves, E.J., Schonmann, R.H.: Behavior of droplets for a class of glauber dynamics at very low temperature. Probab. Theory Relat. Fields 91(3-4), 331-354 (1992)

22. Hollander, Fd., Olivieri, E., Scoppola, E.: Metastability and nucleation for conservative dynamics. J. Math. Phys. 41(3), 1424-1498 (2000) 
23. Den Hollander, F., Nardi, F., Olivieri, E., Scoppola, E.: Droplet growth for three-dimensional Kawasaki dynamics. Probab. Theory Relat. Fields 125(2), 153-194 (2003)

24. Gaudilliere, A., Olivieri, E., Scoppola, E.: Nucleation pattern at low temperature for local Kawasaki dynamics in two dimensions. Markov Process. Relat. Fields 11, 553-628 (2005)

25. Apollonio, V., Jacquier, V., Nardi, F.R., Troiani, A.: Metastability for the ising model on the hexagonal lattice. arXiv preprint arXiv:2101.11894, (2021)

26. Zocca, A.: Tunneling of the hard-core model on finite triangular lattices. Random Struct. Algorithm. 55(1), 215-246 (2019)

27. Cirillo, E.N., Nardi, F.R.: Metastability for a stochastic dynamics with a parallel heat bath updating rule. J. Stat. Phys. 110(1), 183-217 (2003)

28. Cirillo, E.N., Nardi, F.R., Spitoni, C.: Competitive nucleation in reversible probabilistic cellular automata. Phys. Rev. E 78(4), 040601 (2008)

29. Cirillo, E.N., Nardi, F.R., Spitoni, C.: Metastability for reversible probabilistic cellular automata with self-interaction. J. Stat. Phys. 132(3), 431-471 (2008)

30. Procacci, A., Scoppola, B., Scoppola, E.: Probabilistic cellular automata for low-temperature 2-d Ising model. J. Stat. Phys. 165(6), 991-1005 (2016)

31. Dai Pra, P., Scoppola, B., Scoppola, E.: Fast mixing for the low temperature 2d Ising model through irreversible parallel dynamics. J. Stat. Phys. 159(1), 1-20 (2015)

32. Bovier, A., Eckhoff, M., Gayrard, V., Klein, M.: Metastability and low lying spectral in reversible Markov chains. Commun. Math. Phys. 228(2), 219-255 (2002)

33. Bovier, A., Eckhoff, M., Gayrard, V., Klein, M.: Metastability in reversible diffusion processes I. Sharp asymptotics for capacities and exit times. J. Eur. Math. Soc. 6(4), 399-424 (2004)

34. Bovier, A., Den Hollander, F.: Metastability: A Potential-Theoretic Approach, vol. 351. Springer, New York (2016)

35. Cirillo, E.N., Nardi, F.R., Spitoni, C.: Sum of exit times in a series of two metastable states. Eur. Phys. J. Spec. Top. 226(10), 2421-2438 (2017)

36. Bashiri, K.: On the metastability in three modifications of the Ising model. View J. Impact 25(3), 483-532 (2019)

37. Bovier, A., Manzo, F.: Metastability in Glauber dynamics in the low-temperature limit: beyond exponential asymptotics. J. Stat. Phys. 107(3-4), 757-779 (2002)

38. Bovier, A., den Hollander, F., Nardi, F.R.: Sharp asymptotics for Kawasaki dynamics on a finite box with open boundary. Prob. Theory Relat. Fields 135(2), 265-310 (2006)

39. den Hollander, F., Nardi, F., Troiani, A., et al.: Metastability for Kawasaki dynamics at low temperature with two types of particles. Electron. J. Prob. 17, 1-26 (2012)

40. Jovanovski, O.: Metastability for the Ising model on the hypercube. J. Stat. Phys. 167(1), 135-159 (2017)

41. den Hollander, F., Nardi, F.R., Taati, S., et al.: Metastability of hard-core dynamics on bipartite graphs. Electron. J. Prob. 23, 1-65 (2018)

42. Nardi, F., Spitoni, C.: Sharp asymptotics for stochastic dynamics with parallel updating rule. J. Stat. Phys. 146(4), 701-718 (2012)

43. Bet, G., Jacquier, V., Nardi, F.R.: Effect of energy degeneracy on the transition time for a series of metastable states: application to probabilistic cellular automata. arXiv preprint arXiv:2007.08342, (2020)

44. Beltran, J., Landim, C.: Tunneling and metastability of continuous time Markov chains. J. Stat. Phys. 140(6), 1065-1114 (2010)

45. Beltrán, J., Landim, C.: Tunneling and metastability of continuous time Markov chains II, the nonreversible case. J. Stat. Phys. 149(4), 598-618 (2012)

46. Gaudilliere, A., Landim, C.: A Dirichlet principle for non reversible Markov chains and some recurrence theorems. Probab. Theory Relat. Fields 158, 55-89 (2014)

47. Bianchi, A., Gaudilliere, A.: Metastable states, quasi-stationary distributions and soft measures. Stoch. Process. Appl. 126(6), 1622-1680 (2016)

48. Alonso, L., Cerf, R.: The three dimensional polyominoes of minimal area. Electron. J. Combinatorics 3(1), R27 (1996)

Publisher's Note Springer Nature remains neutral with regard to jurisdictional claims in published maps and institutional affiliations. 